DOCUMENT OCCASIONNEL

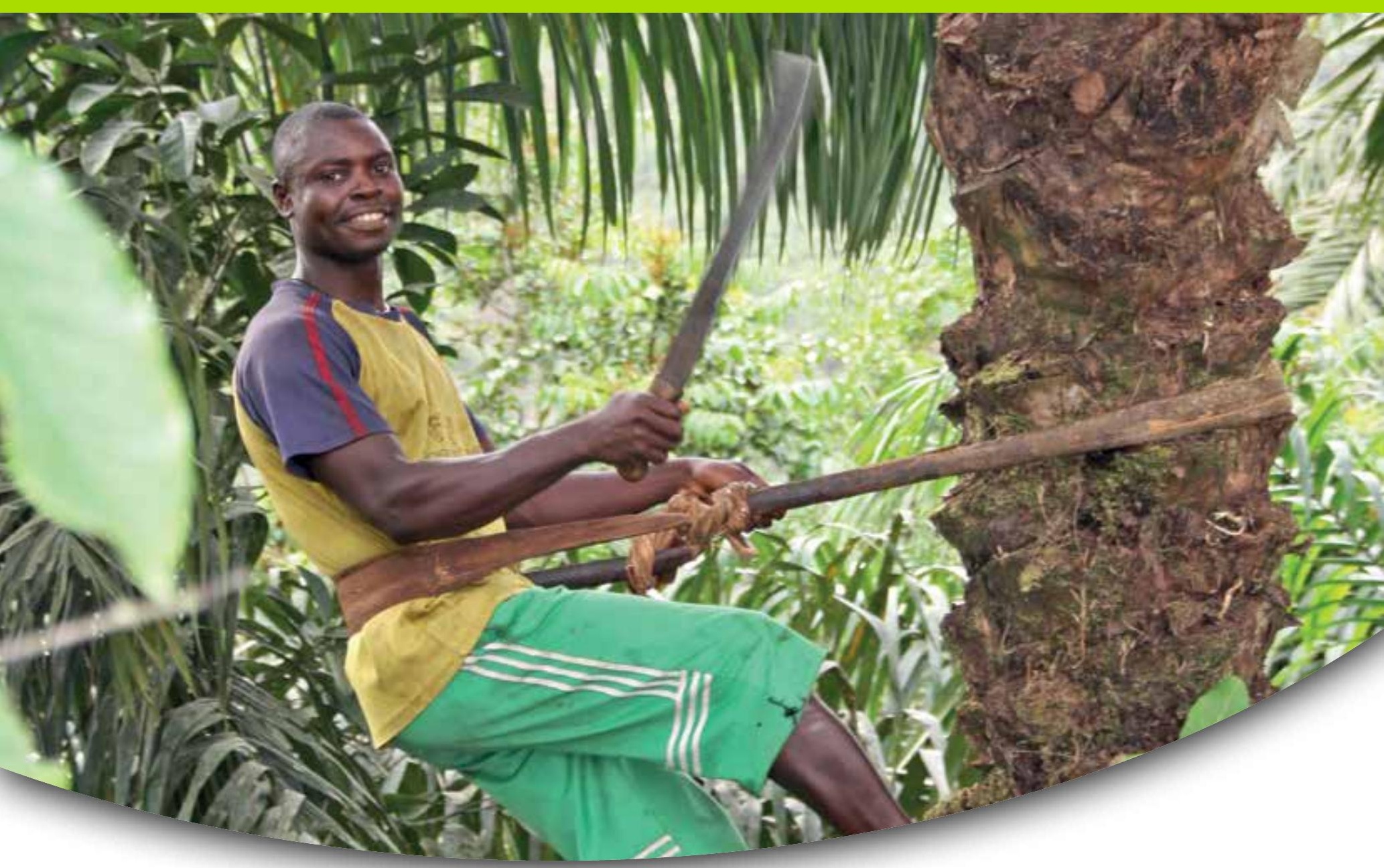

\title{
Historique du secteur palmier à huile au Cameroun
}

Thomas Eric Ndjogui

Raymond Ndip Nkongho

Sylvain Rafflegeau

Laurène Feintrenie

Patrice Levang

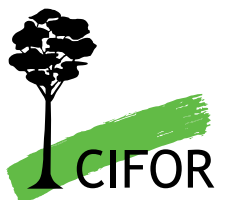





\section{Historique du secteur palmier à huile au Cameroun}

Thomas Eric Ndjogui

Raymond Ndip Nkongho

Sylvain Rafflegeau

Laurène Feintrenie

Patrice Levang 
Document occasionnel 109

(c) 2014 Centre de recherche forestière internationale (CIFOR)

Le contenu de cette publication est soumis à une licence des Creative Commons Attribution-Non CommercialNoDerivs 3.0 Unported License http://creativecommons.org/licenses/by-nc-nd/3.0/

ISBN: 978-602-1504-38-3

Ndjogui TE, Nkongho RN, Rafflegeau S, Feintrenie L et Levang P. 2014. Historique du secteur palmier à huile au Cameroun. Document occasionnel 109. CIFOR, Bogor, Indonésie.

Photo par Patrice Levang

Grimpeur à Fontem, Sud-Ouest Cameroun.

Les auteurs déclarent ne pas avoir de conflits d'intérêts.

Cette étude a été réalisée pour le projet Sustainable Palm Oil Production (SPOP), financé par l'ANR Agrobiosphère et coordonné par le Centre de Coopération Internationale en Recherche Agronomique pour le Développement (CIRAD) en collaboration avec le Centre de recherche forestière internationale (CIFOR), I'Institut de Recherche pour le Développement (IRD) et l'Institut National de la Recherche Agronomique (INRA).

CIFOR

JI. CIFOR, Situ Gede

Bogor Barat 16115

Indonésie

$\mathrm{T}+62(251) 8622-622$

$\mathrm{F}+62(251) 8622-100$

E cifor@cgiar.org

\section{cifor.org}

Nous tenons à remercier tous les donateurs qui ont soutenu cette recherche avec leurs contributions au Fonds du CGIAR. Pour une liste des donateurs au Fonds, s'il vous plaît voir : https://www.cgiarfund.org/FundDonors.

Tous les points de vue figurant dans cet ouvrage sont ceux des auteurs. Ils ne représentent pas forcément les points de vue du CIFOR, des responsables de la rédaction, des institutions respectives des auteurs, des soutiens financiers ou des relecteurs. 


\section{Table des matières}

Avant-propos et remerciements $\quad \mathbf{v}$

Sigles et abréviations $\quad$ vi

Résumé viii

1 Introduction générale : Considérations préliminaires 1

1.1 Rappel de l'histoire du Cameroun 1

1.2 La géographie et la culture du palmier à huile au Cameroun 1

1.3 Les produits du palmier à huile valorisés au Cameroun 5

1.4 Contexte macroéconomique et perspectives de développement de la filière 5

1.5 Méthodologie de notre analyse historique de la filière palmier à huile au Cameroun 6

2 Exploitation ancestrale du palmier à huile au Cameroun $\quad 8$

2.1 Le palmier à huile, une culture ancrée dans la tradition 8

2.2 Les pratiques agricoles ancestrales dans les palmeraies «naturelles " 8

2.3 L'obtention des produits du palmier à huile 9

2.4 Une forme d'exploitation qui résiste à l'épreuve du temps 13

3 Les prémisses du developpement élæicole $\quad 14$

$\begin{array}{lll}3.1 & \text { Les produits du palmier à la veille de la colonisation européenne } & 14\end{array}$

$\begin{array}{ll}3.2 & \text { Les premières plantations de palmier à huile au Cameroun } \\ 3.3 & 14\end{array}$

3.3 La Guerre de 1914-1918 et le début des plantations managériales des colons $\quad 15$

3.4 Une production assurée essentiellement par les palmeraies " naturelles » 22

4 La modernisation du secteur élæicole camerounais $\quad \mathbf{2 4}$

4.1 Une forte implication de l'État dans la modernisation de l'élæiculture 24

4.2 La création des centres de recherche spécialisés 24

4.3 Le programme de plantations familiales de palmiers sélectionnés 24

4.4 Les grandes actions de développement élæicole du Cameroun indépendant 26

4.5 Le FONADER et la mise en œuvre du programme de développement des
plantations villageoises

4.6 La rupture du partenariat entre agro-industries publiques et petits planteurs $\quad 32$

4.7 Un modèle de développement élæicole réussi mais éphémère 34

5 La nouvelle organisation du secteur à partir de $1990 \quad 35$

5.1 Le contexte sociopolitique du Cameroun au début des années $1990 \quad 35$

5.2 Les principaux systèmes de production d'huile de palme au Cameroun 36

5.3 La première transformation des régimes de palme 42

5.4 L'essor de la seconde transformation 44

5.5 L'organisation de la filière palmier à huile 44

5.6 Le retour de l'intervention de l'État 45

5.7 De nombreuses initiatives privées : le projet de villagisation de la SOCAPALM 48

5.8 Les demandes de nouvelles concessions élæicoles 48

5.9 La restructuration et la quête de la durabilité de la filière palmier à huile 49

6 Conclusion générale : Enjeux et perspectives du secteur élæicole camerounais $\quad 50$

7 Bibliographie $\quad 53$ 


\section{Liste des figures, tableaux, photos et encadrés}

\section{Figures}

1 Carte administrative du Cameroun viii

2 Les zones agroécologiques du Cameroun 2

3 L'aire d'extension du palmier à huile au Cameroun $\quad 4$

4 Production mondiale d'huiles végétales en 2011

5 Les flux migratoires allemands au Cameroun (1891-1913) 14

6 Exportations du Cameroun (1904-1905) 15

$7 \quad$ Évolution des cours des produits du palmier à huile entre 1929 et $1934 \quad 17$

8 Évolution des exportations des produits du palmier au Cameroun entre 1920 et $1944 \quad 18$

9 Exportations d'huile de palme et de palmiste au Cameroun en $1935 \quad 18$

10 Évolution des exportations d'huile de palme au Cameroun (1938-1962) 23

11 La demande intérieure en huile de palme au Cameroun (1920-1980) 27

12 Superficies des palmeraies sélectionnées plantées par les petits planteurs et les agro-industries du Cameroun entre 1996 et 2001

13 Production d'huile de palme au Cameroun entre 1964 et 2012

14 Vente des pressoirs artisanaux et production d'huile de palme au Cameroun 43

15 Répartition entre les 3 sites des organisations paysannes de palmier à huile soutenues par le PPDR 46

\section{Tableaux}

$1 \quad$ Les principales plantations coloniales au Cameroun en $1952 \quad 20$

2 Les agro-industries de la subdivision d'Edéa en $1952 \quad 20$

3 Palmeraies européennes monospécifiques de la subdivision d'Eséka dans les années $1950 \quad 20$

4 Plantations coloniales plurispécifiques d'Eséka dans les années $1950 \quad 21$

5 Production d'huile de palme par les agro-industries au Cameroun en $2008 \quad 38$

$6 \quad$ Les petits planteurs encadrés en 1993

7 Caractéristiques de quelques sites de plantations encadrées en $2012 \quad 40$

$8 \quad$ Les principales huileries du Cameroun $\quad 42$

9 La cession d'huile de palme brute aux transformateurs (2004-2007) 43

\section{Photos}

1 Le lemb, cerceau ellipsoïdal à grimper 9

2 Lieu d'extraction traditionnelle de l'huile de palme à Fontem (Sud-Ouest) 10

3 Un palmier déterré puis abattu en coupant les racines 12

4 La sève qui s'écoule du bourgeon apical fermente spontanément en se transformant en vin de palme

\section{Encadrés}

1 Les zones agroécologiques du Cameroun $\quad 3$

2 Le Secteur de Modernisation 25

3 Les objectifs de la Nouvelle Politique Agricole du Cameroun $\quad 37$ 


\section{Avant-propos et remerciements}

Le projet SPOP est financé par l'ANR Agro-

biosphère, coordonné par le CIRAD en collaboration avec le CIFOR, l'IRD et l'INRA. Débuté en 2012, il s'achève en 2014 et couvre le Cameroun et l'Indonésie. Le projet SPOP souhaite contribuer à réduire le manque d'informations sur le palmier à huile dans les sites de mise en œuvre. Pour ce faire, la démarche intègre une analyse fine des impacts des systèmes de culture variés du palmier à huile à l'échelle de la plantation couplée à une analyse des dynamiques d'utilisation des sols à l'échelle territoriale. Aussi, les principaux objectifs du projet SPOP sont :

- d'analyser l'influence des changements globaux sur les divers systèmes de culture du palmier à huile,

- d'identifier les obstacles et opportunités, ainsi que les incertitudes liées aux possibles transitions vers des systèmes durables, et

- d'élaborer des stratégies et des instruments pour faciliter ces transitions.

À terme, le projet SPOP entend fournir de nouvelles connaissances et données sur les impacts socioéconomiques et environnementaux des systèmes de culture du palmier à huile, tout en développant des outils innovants pour évaluer ces impacts dans l'optique de développement des systèmes durables (http://www.gred.ird.fr/ programmes-de-recherche/projets-anr/spop). La nécessité de ressortir l'état des lieux de la filière palmier à huile au Cameroun justifie la conduite de la présente étude.

Les auteurs remercient les organismes bailleurs de fonds, en l'occurrence le CIRAD et le CIFOR pour le financement de cette étude. Ils expriment également leur gratitude envers les membres du gouvernement, notamment le MINADER, le personnel des agro-industries et des industries de seconde transformation de l'huile de palme au Cameroun sans oublier l'UNEXPALM et les populations locales qui ont donné de leur temps et de leur savoir durant les visites sur le terrain et fourni des informations utiles pour la réalisation de ce travail.

Les auteurs sont particulièrement redevables envers Emmanuel Ngom pour ses nombreux conseils et informations, et Cécile Bessou pour ses observations sur une version antérieure de ce rapport. 


\title{
Sigles et abréviations
}

\author{
AGTA \\ AID \\ ANY \\ APROCOM-Ph \\ ATPO \\ BCD \\ BEI \\ BIRD \\ CAMDEV \\ CCC \\ CChC \\ CDC \\ CED \\ CEDAC \\ CEREPAH \\ CFEC \\ CIFOR \\ CIRAD \\ CLIP \\ CNPS \\ CRAT \\ DSCE \\ DSDSR \\ DWH \\ FAO \\ FED \\ FIDES \\ FMI \\ FONADER \\ GAM \\ HVC \\ IRAD \\ IRD \\ IRHO \\ MINADER \\ MINCOMMERCE \\ MINDAF \\ MINEFI \\ MINFOF \\ MINMIDT \\ MINEPAT \\ MINRESI \\ NPAC \\ OMC \\ ONU \\ Agence d'Aménagement et de Gestion des Terres Agricoles \\ Association Internationale de Développement \\ Archives Nationales de Yaoundé \\ Amélioration de la Productivité et de la Compétitivité de la filière Palmier à Huile \\ en Afrique centrale et de l'Ouest \\ Association des Transformateurs des Produits Oléagineux \\ Banque Camerounaise de Développement \\ Banque Européenne d'Investissement \\ Banque Internationale pour la Reconstruction et le Développement \\ Cameroons Development \\ Complexe Chimique Camerounais \\ Compagnie Commerciale Chypriote \\ Cameroon Development Corporation \\ Centre pour l'Environnement et le Développement \\ Centre de Développement Auto-Centré \\ Centre Spécialisé de Recherche sur Palmier à Huile de la Dibamba \\ Congrégation des Frères des Écoles chrétiennes \\ Centre de recherche forestière internationale \\ Centre de coopération internationale en recherche agronomique pour \\ le Développement \\ Consentement Libre, Informé et Préalable \\ Caisse Nationale de Prévoyance Sociale \\ Centre Rural d'appui Technique \\ Document de Stratégie pour la Croissance et l'Emploi \\ Document de Stratégie de Développement du Secteur Rural \\ Deutsche Westafrikanische Handelsgesellschaft \\ Organisation des Nations Unies pour l'Alimentation et l'Agriculture \\ Fonds européen de développement \\ Fond International pour le Développement Économique et Social des Territoires \\ d'Outre-Mer \\ Fond Monétaire International \\ Fond National de Développement Rural \\ Groupements d'Agriculteurs Modernes \\ Haute Valeur de Conservation \\ Institut de Recherche Agricole pour le Développement \\ Institut de Recherche pour le Développement \\ Institut de Recherche pour les Huiles et Oléagineux \\ Ministère de l'Agriculture et du Développement Rural \\ Ministère du Commerce \\ Ministère des Domaines et Affaires Foncières \\ Ministère des Finances \\ Ministère des Forêts et des la Faune \\ Ministère des Mines, de l'Industrie et du Développement Technologique \\ Ministère de l'Économie, de la planification et de l'aménagement du Territoire \\ Ministère de la Recherche Scientifique et de l'Innovation \\ Nouvelle Politique Agricole du Cameroun \\ Organisation Mondiale du Commerce \\ Organisation des Nations Unies
}


ONUDI

PACA

PALMCAM

PDPV

PLANOPAC

PNPSPHS

PPDR

PPTE

REDD

RSPO

SAFA

SAFACAM

SCR-MAYA

SDN

SEMMaritime

SFC

SMP

SNC

SNPHPC

SOCAPALM

SODECAO

SOFINC

SOMUDER

SOPACOR

SOPAME

SOSUCAM

SPFS

SPOP

SPROA

UAC

UNEXPALM

WWF
Fond des Nations Unies pour le Développement Industriel

Programme d'Amélioration de la Compétitivité Agricole

Palmeraies du Cameroun

Programme de Développement des Palmeraies Villageoises

Plateforme Nationale des Organisations de Producteurs Agrosylvopastoraux

du Cameroun

Programme National de Production des Semences de Palmier à Huile Sélectionnées

Projet Pôle de Développement Rural

Pays Pauvres Très Endettés

Réduction des Émissions dues au Déboisement et à la Dégradation Forestière

Roundtable for Sustainable Palm Oil

Société Africaine Forestière et Agricole

Société Africaine Forestière et Agricole du Cameroun

Société Camerounaise de Raffineries Maya

Société Des Nations

Secteur Expérimental de Modernisation et d'Action Rurale pour le Littoral

Société Financière et Commerciale

Secteur de Modernisation du Palmier

Société Nationale du Cameroun

Syndicat National des Producteurs d'Huile de Palme du Cameroun

Société Camerounaise de Palmeraies

Société de Développement du Cacao

Société Financière des Caoutchoucs

Sociétés Mutuelles de Développement Rural

Société des Palmeraies du Cameroun oriental

Société des palmeraies de M'Bongo et d'Eséka

Société Sucrière du Cameroun

Société des Palmeraies de la Ferme Suisse

Projet Sustainable Palm Oil Production

Société des Plantations Réunies de l'Ouest Africain

United Africa Company

Union des Exploitants de Palmiers à huile du Cameroun

Fond Mondial pour la Nature 


\section{Résumé}

Le palmier à huile est une plante tropicale originaire du golfe de Guinée. Il pousse spontanément dans toute la zone forestière $\mathrm{du}$ Cameroun où il a longtemps fait l'objet d'une exploitation artisanale. C'est au début du XIX ${ }^{\mathrm{e}}$ siècle, entre 1904 et 1907, que sont créées les premières plantations coloniales. Après une longue période de stagnation marquée par la Première Guerre mondiale, la crise de 1929 et la Deuxième Guerre mondiale, la culture du palmier à huile entre dans une phase de modernisation avec la création de la station IRHO de La Dibamba en 1947, la création de la SOCAPALM en 1968, la construction d'usines industrielles et la mise en œuvre d'un vaste programme de développement

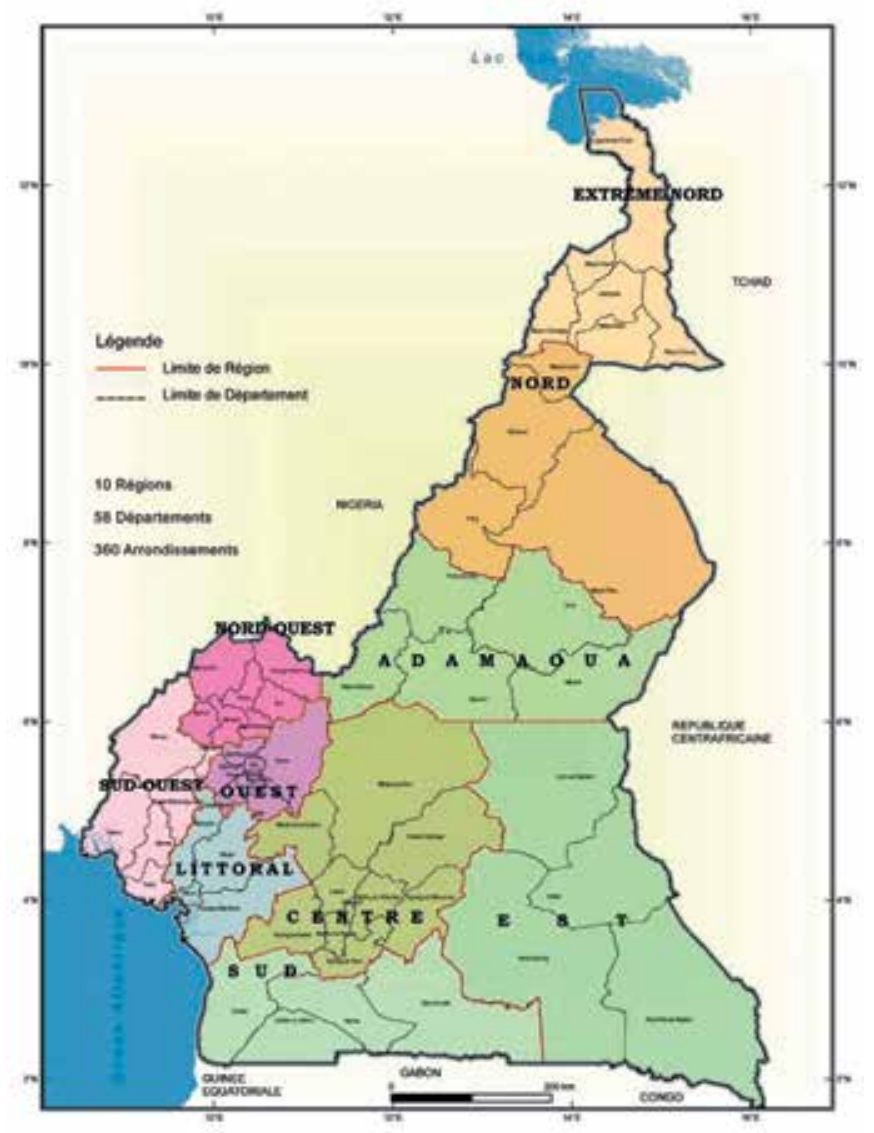

Figure 1. Carte administrative du Cameroun Source : Institut National de Cartographie, Atlas national de développement physique du Cameroun, 2011 des plantations villageoises pendant les années 1970 et 1980 avec l'appui du Fond National de Développement Rural (FONADER) créé en 1973. Cependant, la crise des années 1980 entraîne la faillite du FONADER en 1990, l'arrêt du programme de plantations villageoises, la rupture du partenariat entre les planteurs villageois et les agro-industries et le désengagement de l'État suite à la mise en œuvre des programmes d'ajustement structurel. On assiste ainsi dès le début des années 1990 à de multiples recompositions caractérisées entre autres par la reconfiguration $\mathrm{du}$ partenariat entre les planteurs villageois et les agro-industries, l'émergence de nouveaux sites de développement du palmier, le développement spontané des palmeraies villageoises non encadrées et des presses artisanales, autre débouché possible pour les régimes des petits planteurs. Cette dynamique de production primaire portée par des investissements privés de ruraux et d'urbains pour créer des palmeraies villageoises est soutenue en aval par le développement de l'industrie de la seconde transformation, qui, en se diversifiant, augmente la demande intérieure en huile de palme en association avec la croissance démographique et l'élévation du niveau de vie des populations. Depuis les années 2000, la filière est marquée par la privatisation de la SOCAPALM et sa reprise par le groupe français Bolloré qui contrôle également la SAFACAM et la SPFS, le retour de l'assistance de l'État aux planteurs villageois avec la création du Programme de Développement des Palmeraies Villageoises (PDPV) et l'arrivée annoncée de nouvelles agro-industries qui négocient des concessions avec l'État. Le souci de la durabilité de la filière amène les acteurs à initier la rédaction d'une stratégie nationale de développement durable du palmier à huile sous la coordination du MINADER. Dans ce contexte particulièrement dynamique, une attention particulière doit être accordée aux ayants droit des terres que l'État pourrait attribuer aux agro-industries, aux petits planteurs et aux relations entre ces derniers et les agro-industries dans la perspective d'un partenariat gagnant-gagnant. 


\section{Introduction générale : Considérations préliminaires}

\subsection{Rappel de I'histoire du Cameroun}

Logé au cœur du golfe de Guinée, le Cameroun est un pays de l'Afrique centrale. Il s'étend entre $1^{\circ} 40$ et $13^{\circ}$ de latitude nord et $8^{\circ}$ et $16^{\circ}$ de longitude est. Le pays couvre une superficie de 475442 $\mathrm{km}^{2}$ (fig. 1). Avant la colonisation européenne qui intervient à la fin du XIX ${ }^{\mathrm{e}}$ siècle, le territoire camerounais n'est qu'une mosaïque de peuples et de tribus ayant une organisation sociopolitique diversifiée. Le 14 juillet 1884 marque le début du protectorat allemand au Cameroun et la naissance du territoire camerounais limité à cette époque à la seule ville de Douala, son centre originel, avant de s'étendre ensuite aux frontières actuelles. En 1914, la Première Guerre mondiale éclate et en 1916, les Allemands sont chassés du Cameroun par les Anglais et les Français. Dès le 4 mars 1916, les deux puissances signent une convention de partage du territoire camerounais en attendant l'approbation de la communauté internationale. La France obtient les 4/5 de la partie orientale du territoire tandis que le $1 / 5$ restant situé à l'Ouest est confié à la Grande-Bretagne. Le Cameroun devient un condominium anglo-français jusqu'à la fin de la guerre en 1918. Le 28 juin 1919, le traité de Versailles est signé et oblige l'Allemagne à renoncer à toute revendication sur son ancien protectorat. Le 10 juillet 1919, la Société des Nations (SDN) qui vient d'être créée entérine le partage du Cameroun selon le traité anglo-français de 1916. La partie occidentale anglaise est formée de deux territoires disjoints appelés Cameroun du Nord et Cameroun du Sud. En 1920, les autorités coloniales anglaises décident d'administrer les deux Cameroons comme une partie intégrante du Nigéria au lieu d'en faire un territoire autonome. Le régime de mandat prend fin en 1945 avec la dissolution de la SDN et la création de l'Organisation des Nations Unies (ONU). Le Cameroun devient alors un territoire sous tutelle de l'ONU mais toujours administré par la Grande-Bretagne et la France. Après des épisodes sanglants d'émancipation, notamment en Sanaga-Maritime et dans la région de l'Ouest, le Cameroun sous tutelle française proclame son indépendance le $1^{\text {er }}$ janvier 1960. En 1961, un référendum a lieu dans l'ensemble $\mathrm{du}$ Cameroun sous administration anglaise. Le Cameroun du Nord opte pour le rattachement au Nigéria tandis que son homologue du Sud vote pour le rattachement à l'ex-Cameroun sous administration française. Le $1^{\text {er }}$ Octobre 1961, le Cameroun sous tutelle anglaise obtient son indépendance : c'est la naissance de la République fédérale du Cameroun. À la faveur de cette unification, l'ex-Cameroun sous administration française devient le Cameroun oriental tandis que celui sous administration anglaise prend le nom de Cameroun occidental.

Puis, les deux États fédérés s'unissent de nouveau le 20 mai 1972 pour former la République Unie du Cameroun qui devient la République du Cameroun en 1984.

\subsection{La géographie et la culture du palmier à huile au Cameroun}

De par sa situation géoclimatique caractérisée par son relief et son étirement en latitude ainsi que des entrées d'air maritime humide au sud et d'air sec sahélien au nord, le Cameroun est une mosaïque de milieux naturels qui constituent autant d'atouts pour le développement agricole. La diversité des conditions pédo-climatiques permet de subdiviser le territoire national en 5 grandes zones agro-écologiques (fig. 2 ; encadré 1).

\subsubsection{Les exigences pédo-climatiques du palmier à huile}

Les meilleures conditions pédo-climatiques pour la culture du palmier à huile sont les suivantes (Hartley, 1988 ; Hoyle et Levang, 2012): 
- des températures élevées toute l'année, entre 25 et $28^{\circ} \mathrm{C}$ avec des minima supérieurs à $18^{\circ} \mathrm{C}$ pour les mois les plus froids ;

- un ensoleillement d'au moins 5 heures/jour;

- des précipitations comprises entre 1800 et $2400 \mathrm{~mm} / \mathrm{an}$. Toutefois, une sécheresse prolongée de plus de 90 jours est un sérieux handicap au développement optimal du palmier à huile ;
- des précipitations excédentaires sont bien tolérées tant que les sols sont bien drainés ;

- des sols riches et bien drainés même si le palmier se satisfait de sols pauvres si la fertilisation est assurée ;

- une altitude basse, idéalement inférieure à $500 \mathrm{~m}$. Les terrains plats sont plus indiqués pour éviter l'érosion du sol et faciliter la récolte et le transport des fruits.

Zone forestière mono-modale

Superficie : $45658 \mathrm{~km}^{2}$

Pluviometrie : 2500 a $4000 \mathrm{~mm} / \mathrm{an}$, règime monomodal

Sols : pentes volcaniques, sédiments dorigine rocheuse le long de la cote

Cultures : cacao, banane, café, plantain, huile de palme, gingembre, poivre

\section{Zone forestière bi-modale}

Superficie : $165770 \mathrm{~km}^{2}$

Pluviometrie : 1500 a $2000 \mathrm{~mm} / \mathrm{an}, 2$ saisons humides distinctes

Sols : ferraintiques, acides, argileux, faible capacité de rétention des eléments nutritifs

Cultures : cacao, cafe, manioc, plantain, mais, huile de palme, ananas

Zone des hauts plateaux

Superficie : $31192 \mathrm{~km}^{2}$

Pluviométrie : 1500 à $2000 \mathrm{~mm} / \mathrm{an}, 180 \mathrm{jrs}$ de pluie

Sols : très fertiles et propices aux activités agricoles, jeunes sur fortes pentes. lessivés dans les vieux plateaux, horizon $B$ dilluviation dans dépressions ferm plateaux enrichis en materiaux volcaniques

Cultures : cacao, caft, mails, haricot sec, pomme de terre, maraichage

Zone des hautes savanes

Superficie : $123077 \mathrm{~km}^{2}$

Pluviométrie : $1500 \mathrm{~mm} / \mathrm{an}, 150$ jrs de pluie

Sols : perméables, capacité de rétention d'eau moyenne. sols ferralitiques bruns ou rouges et sols hydromorphes

Cultures : mails, coton, mil-sorgho, igname, pomme de terre

\section{Zone soudano-sahélienne}

Superficie : $100353 \mathrm{~km}^{2}$

Pluviometrie : 400 a $1200 \mathrm{~mm} / \mathrm{an}$

Sols : grande diversite : ferrugineux, lessivés.

hydromorphes, alluvionaires, lithosols, vertisols etc.

Cultures : coton, mil-sorgho, niébé, oignon, sésame

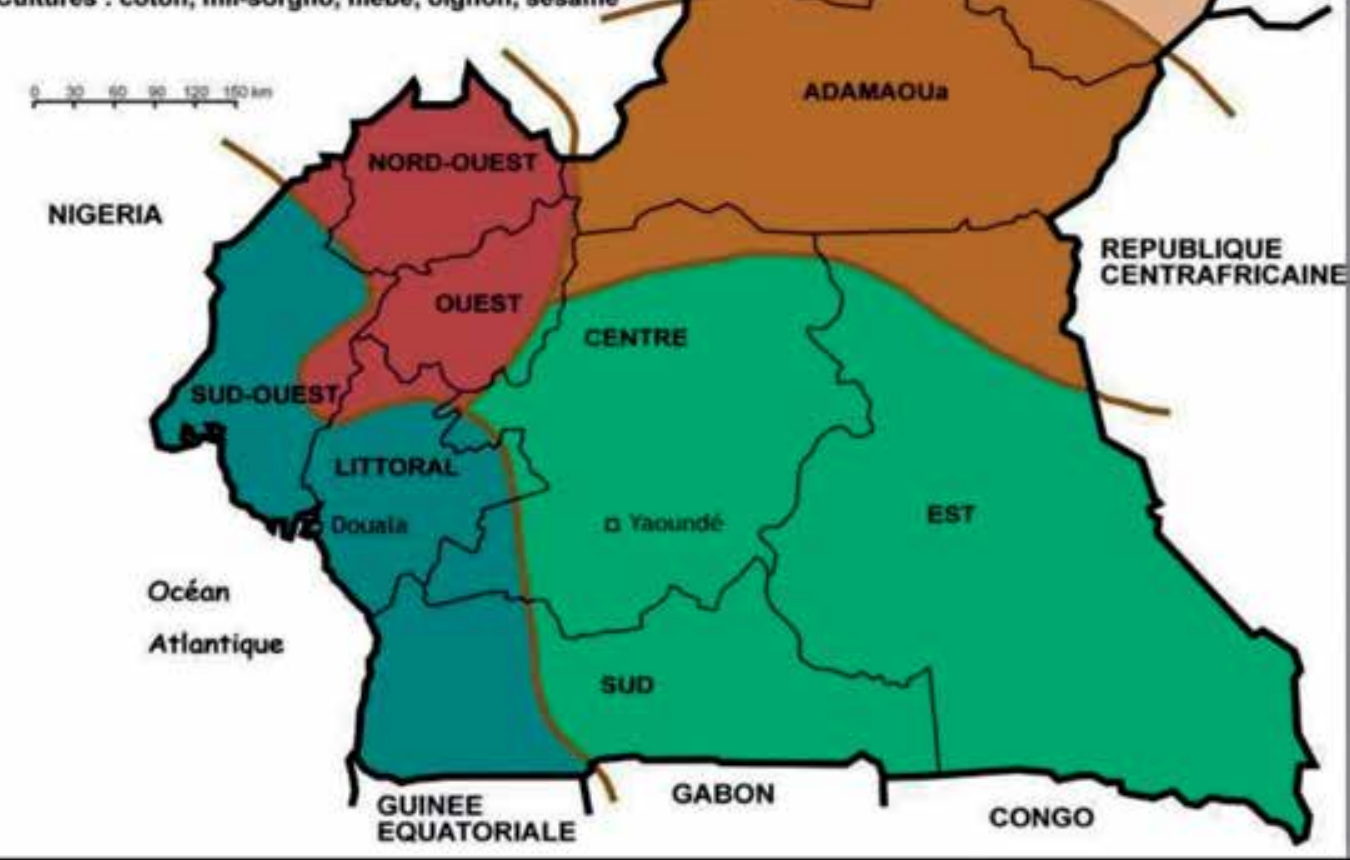

Figure 2. Les zones agroécologiques du Cameroun 


\section{Encadré 1 : Les zones agroécologiques du Cameroun}

\section{La zone de forêts denses humides à pluviométrie monomodale (zone I)}

La zone de forêts denses humides à pluviométrie monomodale est comprise entre $2^{\circ} 6^{\prime \prime}$ et $6^{\circ} 12^{\prime \prime}$ de latitude nord, et $8^{\circ} 48^{\prime \prime}$ et $10^{\circ} 30^{\prime \prime}$ de longitude est. Elle intéresse les régions du Littoral et du Sud-Ouest, ainsi que la bordure côtière de la région du Sud. Elle s'étend sur une superficie de 4,5 millions d'hectares dont 282000 (soit 6,3\%) sont cultivés. II s'agit essentiellement d'une zone de basse altitude dont la monotonie est rompue par le Mont Cameroun qui culmine à $4095 \mathrm{~m}$. Ici règne un climat équatorial de mousson, très humide, chaud et pluvieux. Le régime pluviométrique est de type monomodal. Les pluies sont abondantes et réparties sur toute l'année. Elles varient en moyenne entre 2500 à $4000 \mathrm{~mm}$, à l'exception de la localité de Debundscha qui s'illustre parmi les régions les plus pluvieuses du monde avec $11000 \mathrm{~mm}$ d'eau par an. Les températures sont élevées tout au long de l'année et oscillent entre 22 et $29^{\circ} \mathrm{C}$. Le taux d'humidité de l'air est compris entre 85 et $90 \%$. Ici prédomine la végétation de forêts denses humides sous plusieurs types de couverts. C'est la zone de prédilection des tubercules et d'une variété de cultures d'exportation en l'occurrence caféier Robusta, cacao, théier, bananier et bananier plantain, palmier à huile, et hévéa.

\section{La zone de forêts humides à pluviométrie bimodale (zone II)}

Cette zone est comprise entre $2^{\circ} 6^{\prime \prime}$ à $4^{\circ} 54^{\prime \prime} / 5^{\circ} 48^{\prime \prime}$ de latitude nord et $10^{\circ} 30^{\prime \prime}$ à $16^{\circ} 12^{\prime \prime}$ de longitude est. Elle couvre presque tout le plateau sud-camerounais entre 500 et $1000 \mathrm{~m}$ d'altitude et intègre les régions du Centre, du Sud et de l'Est sur une superficie totale de 22,5 millions d'hectares. Le climat est de type équatorial guinéen caractérisé par des températures élevées avec les moyennes annuelles de $25^{\circ} \mathrm{C}$ et une pluviométrie de 1500-2000 mm par an. La végétation est essentiellement composée de forêts denses sempervirentes et semi-décidues. Cette zone est plus favorable au développement des cultures pérennes (cacao, caféier robusta, divers arbres fruitiers) et annuelles et pluriannuelles (bananier plantain, canne à sucre, maïs, tabac, cultures maraîchères, tubercules, etc.).

\section{La zone des hauts plateaux de l'Ouest (zone III)}

Cette zone est comprise entre $4^{\circ} 54^{\prime \prime}$ à $6^{\circ} 36^{\prime \prime}$ de latitude nord et $9^{\circ} 18^{\prime \prime}$ à $11^{\circ} 24^{\prime \prime}$ de longitude est et intègre les régions de l'Ouest et du Nord-Ouest sur une superficie totale de 3,1 millions d'hectares. C'est une région de plateaux dont les principaux sont le plateau Bamoun, le plateau Bamiléké et le plateaux volcaniques de Bamenda. Le climat est de type équatorial d'altitude caractérisé par deux saisons d'inégales longueurs. L'altitude abaisse les températures moyennes $\left(19^{\circ} \mathrm{C}\right.$ en moyenne annuelle), les pluies sont abondantes et varient entre $1500-2000 \mathrm{~mm}$ par an. C'est le domaine des savanes et des forêts-galeries. Cette zone est favorable à une mosaïque de cultures incluant caféier Arabica, théier, bananier, maïs, arachide, riz, cultures maraîchères, etc.

\section{La zone des hautes savanes guinéennes (zone IV)}

Cette zone est comprise entre $5^{\circ} 42^{\prime \prime}$ à $8^{\circ} 36^{\prime \prime}$ de latitude nord, et $11^{\circ} 24^{\prime \prime}$ à $14^{\circ} 36^{\prime \prime}$ de longitude est. Sur le plan administratif, elle couvre les régions du Nord, de l'Adamaoua ainsi que la partie septentrionale des départements du Mbam dans la région du Centre et du Lom-et-Djerem dans la région de l'Est, sur une superficie totale de $138000 \mathrm{~km}^{2}$. Elle est constituée dans sa grande partie par un vaste plateau d'altitudes comprises entre 900 et $1500 \mathrm{~m}$, avec des sommets atteignant $1800 \mathrm{~m}$. Le climat est de type tropical soudanien à deux saisons par an. La pluviométrie moyenne annuelle est de l'ordre de $1500 \mathrm{~mm}$. Du fait de l'altitude, les températures sont modérées, avec des moyennes mensuelles de l'ordre de 20 à $26^{\circ} \mathrm{C}$. C'est la zone de prédilection du maïs, du mil, du sorgho et de l'arachide, ainsi que des ignames.

\section{La zone soudano-sahelienne (zone V)}

Cette zone est comprise entre $8^{\circ} 36^{\prime \prime}$ à $12^{\circ} 54^{\prime \prime}$ de latitude nord, et $12^{\circ} 30^{\prime \prime}$ à $15^{\circ} 42^{\prime \prime}$ de longitude est. Adminidtrativement, elle recouvre les régions du Nord et de l'Extrême Nord, soit une superficie de 10,2 millions d'hectares. La pluviométrie est de type monomodale et augmente du nord vers le sud. Elle varie entre 400 et $1200 \mathrm{~mm}$ par an du nord au sud. Contrairement aux précipitations, les températures quant à elles diminuent du nord vers le sud. Les températures moyennes annuelles estimées atteignent $28^{\circ} \mathrm{C}$ à Garoua. Les sols sont essentiellement constitués de vertisols lithomorphes associés aux sols vertiques et des sols ferrugineux plus ou moins lessivés. La végétation est essentiellement faite de steppes à épineux, des prairies périodiquement inondées appelées " yaérées.» Les cultures propices pour cette zone incluent entre autre le sorgho, le mil, le coton, le maïs, le riz, l'arachide, le niébé et les cultures maraîchères. 
- l'humidité moyenne relative mensuelle doit être supérieure à $75 \%$.

\subsubsection{L'aire d'extension du palmier à huile au Cameroun}

Le palmier se développe essentiellement dans le domaine forestier du Sud-Cameroun. Cette zone de prédilection du palmier se subdivise en deux principales aires (fig. 3). La zone à la fois méridionale et océanique couvre les systèmes d'exploitation actuels du palmier à huile au Cameroun. Il s'agit du secteur maritime et côtier comprenant les régions du Sud-Ouest et $\mathrm{du}$ Littoral et la partie occidentale des régions du Centre et du Sud. Il se rattache à la zone agro-écologique de " forêts denses humides à pluviométrie monomodale ». Cette zone est le principal bassin de production d'huile de palme du Cameroun et héberge toutes les agro- industries actuelles du pays et l'essentiel des palmeraies villageoises.

A côté de cette ceinture du palmier à huile, on trouve une zone de développement diffus qui couvre les régions du Centre, du Sud, de l'Est, de l'Ouest, du Nord-Ouest et le sud de l'Adamaoua. Dans ces régions, les conditions de développement du palmier à huile ne sont pas optimales, mais le palmier à huile fait partie des systèmes agraires par son exploitation traditionnelle ancestrale et par l'implantation récente et sporadique des palmeraies villageoises. Certaines régions à l'instar du Sud connaissent une forte expansion de la culture du palmier à huile portée essentiellement par les élites urbaines. Cette région pourrait jouer un rôle déterminant dans l'expansion du palmier à huile au Cameroun du fait de conditions climatiques proches de celles de la zone méridionale et océanique.

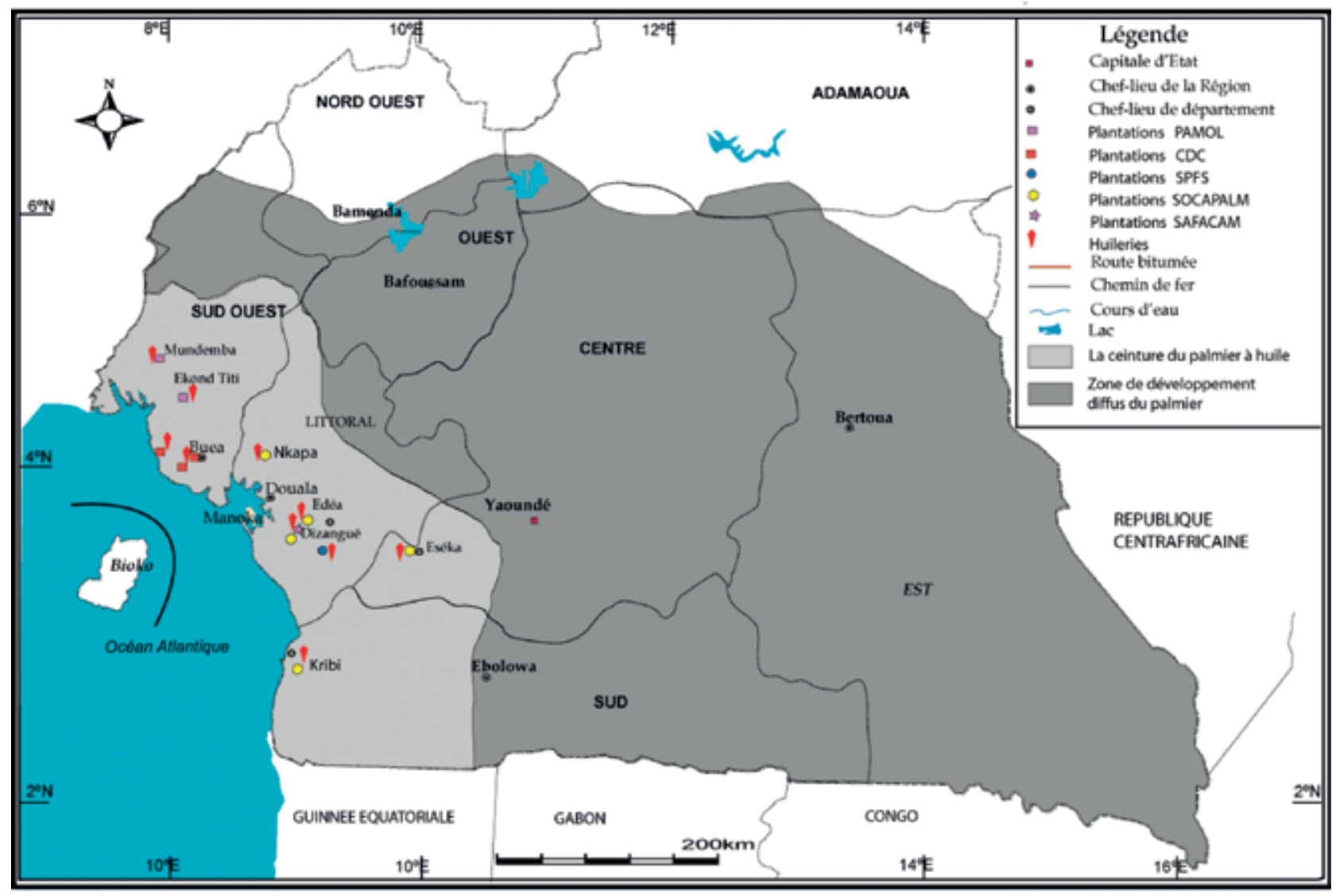

Figure 3 : L'aire d'extension du palmier à huile au Cameroun

Source : INC, 2007 ; IRAD, 2008. 


\subsection{Les produits du palmier à huile valorisés au Cameroun}

Au Cameroun, le palmier à huile est principalement cultivé et exploité pour les deux huiles que l'ont peut extraire de ses fruits de manière industrielle ou artisanale :

- l'huile de palme extraite du péricarpe, la pulpe des fruits ;

- l'huile de palmiste issue de l'endocarpe, l'amande.

Les autres produits incluent :

- le vin de palme, produit exclusivement de manière artisanale ;

- les tourteaux des presses artisanales sont des aliments pour le bétail, mais servent aussi de combustible sur les chantiers d'extraction artisanaux, tandis que dans les huileries industrielles, les fibres sont le combustible des chaudières qui produisent de la vapeur utilisée pour la cuisson des régimes et la production d'électricité alimentant l'huilerie, les bureaux et les villages alentours.

L'industrie de la seconde transformation, principalement constituée de raffineries et de savonneries transforme essentiellement de l'huile de palme industrielle mais aussi artisanale, et dans de moindres proportions de l'huile de palmiste industrielle, afin de vendre une variété de produits alimentaires et cosmétiques sur le marché national et régional. En effet, chaque étape du raffinage donne lieu à des produits commercialisables. Ainsi, le prétraitement (floculation - blanchiment filtration - polissage) donne de l'huile prétraitée tandis que le fractionnement (réchauffage refroidissement - filtration) permet de séparer des corps gras caractérisés par des points de fusions différents : l'oléine, une huile fluide, et la stéarine, une huile concrète. Notons enfin que le palmier à huile offre aux communautés locales de nombreux services sociaux et culturels allant de l'alimentation à la pharmacopée traditionnelle en passant par des matériaux de construction, concourant ainsi au bien-être des populations locales et à leur développement socioculturel.

\subsection{Contexte macroéconomique et perspectives de développement de la filière}

L'huile de palme est devenue le principal produit du palmier à huile échangé à l'échelle mondiale. Elle est la plus importante des huiles végétales devant le soja, le colza et le tournesol (Oil World 2013). Ce dynamisme est principalement porté par

Secteur des plantes oléagineuses (millions de tonnes)

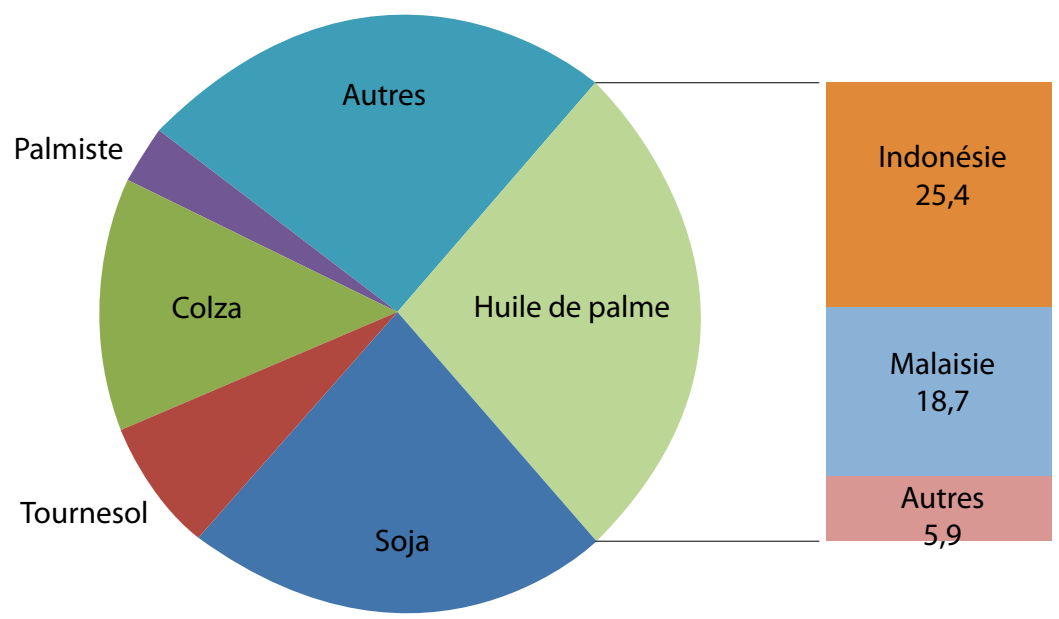

Figure 4 : Production mondiale d'huiles végétales en 2011

Source : Hoyle et Levang, 2012 ; Oil World 2013 
l'Indonésie et la Malaisie qui représentent $85 \%$ de la production mondiale d'huile de palme (fig. 4).

La place du Cameroun dans l'offre mondiale en huile de palme ne fait que régresser. Placé $11^{\mathrm{e}}$ en 2008 avec 160000 t/an d'huile de palme (FAO, 2008), le pays occupe la $13^{\mathrm{e}}$ place en 2012 avec une production de 230000 t/an (Hoyle et Levang, 2012 ; Oil World 2013).

Pourtant, le Cameroun bénéficie de nombreux atouts qui pourraient lui permettre d'être autosuffisant en huile de palme et de gagner des parts de marché mondial et surtout régional.

Pour accroître sa production en huile de palme, le pays peut augmenter la productivité des surfaces déjà plantées et la superficie du verger national en implantant dans de bonnes conditions des palmeraies sélectionnées, tout en contribuant au développement socioéconomique des masses paysannes et en minimisant la dégradation de l'environnement. Au cours de cette dernière décennie, le Gouvernement a pris des mesures de relance de la filière palmier à huile notamment avec la mise en place d'un Programme de Développement des Palmeraies Villageoises (PDPV). Il existe parallèlement à ce projet qui aide les plus démunis à implanter leur premier hectare de palmeraie sélectionnée, des initiatives privées de ruraux ainsi que d'urbains qui implantent euxmêmes et sans l'aide d'un projet, des palmeraies villageoises. Malgré ce projet et cette dynamique spontanée, le déficit de production d'huile de palme au Cameroun reste élevé et ne cesse de croître tout en occasionnant des pénuries récurrentes et une perte de devises pour l'État du fait des importations suscitées. Le déficit est estimé à 85000 t en 2013 contre 50000 t en 2012 (MINADER, 2013), alors qu'avec un potentiel de production avéré et incontestable, l'augmentation de la production du Cameroun reste globalement insuffisante par rapport aux besoins pour les raisons suivantes :

- Les faibles rendements d'une partie des plantations villageoises et industrielles existantes ;

- Le taux d'extraction très bas des unités artisanales, mais aussi d'huileries des agroindustries qui n'ont pas trouvé de repreneurs privés et manquent d'entretien pour garder leurs performances ;
- La faible utilisation de fertilisants en raison de prix très élevés sur le marché local ;

- Le vieillissement des plantations ;

- L'utilisation insuffisante du matériel végétal sélectionné ;

- Les difficultés de transport et de commercialisation de la production pour ceux qui sont loin des huileries, des axes routiers;

- La mauvaise organisation des producteurs villageois pour s'approvisionner en semences sélectionnées, conduire des pépinières, acheter une presse en commun, obtenir des aides et du conseil technique ;

- Le manque d'appui technique en dehors des bassins d'approvisionnement des huileries industrielles.

Le Cameroun entend renverser cette tendance à travers son nouveau programme de développement du palmier à huile décliné dans la Stratégie de Développement du Secteur Rural (SDSR) et le Document de Stratégie pour la Croissance et l'Emploi (DSCE) publiés respectivement en 2005 et en 2009. Le gouvernement ambitionne de faire passer la production d'huile de $177000 \mathrm{t}$ en 2005 à $350000 \mathrm{t}$ en 2020 (DSCE, 2009). Par ailleurs, la mise en place des plans de développement des produits du terroir incluant les farines locales, le sucre, l'huile de palme, la banane plantain, le maïs, le cacao et le coton est prévue. C'est dans ce sillage que le MINADER a réuni les parties prenantes de la filière palmier à huile afin d'élaborer une stratégie nationale de développement durable du palmier à huile. Les discussions engagées en 2012 ont abouti à la création d'un comité multipartite chargé de l'élaboration d'une stratégie nationale de développement durable du palmier à huile au Cameroun par Décision N ${ }^{\circ} 00250 / \mathrm{CAB} /$ MINADER/du 29 juillet 2013. Parmi les missions assignées à ce comité, figure le diagnostic actualisé de la filière palmier à huile au Cameroun.

\subsection{Méthodologie de notre analyse historique de la filière palmier à huile au Cameroun}

La présente analyse de l'historique de la filière palmier à huile au Cameroun rentre dans une démarche d'actualisation du diagnostic de la filière, et vise à améliorer la compréhension des principales dynamiques observées récemment dans la filière. 
Ce rapport est réalisé sur la base de recherches documentaires menées au sein des bibliothèques universitaires (Buéa, Douala, et Yaoundé I) et des administrations (MINADER, MINEPAT) camerounaises concernées par le développement du palmier à huile. De nombreux documents ont été également obtenus par le biais d'internet et des experts en élæiculture. Les documents consultés incluent les rapports de stages, les mémoires de maîtrise et de DEA, des thèses et les rapports d'études sur l'agriculture en général et le palmier à huile en particulier. Les enquêtes de terrain des doctorants (Raymond Ndip NKONGHO dans le Sud-Ouest et Thomas Eric NDJOGUI dans la Sanaga-Maritime) impliqués dans la réalisation de ce travail ont également été mises à contribution de même que leur propre expertise sur le palmier. Ces enquêtes de terrain s'appuient essentiellement sur des entretiens semi-directifs auprès des experts sur le palmier et des acteurs du secteur palmier à huile au Cameroun en l'occurrence les petits planteurs, les agro-industries, l'UNEXPALM, le coordonnateur du PDPV, le MINADER, etc. Les sites d'enquêtes incluent le Sud-Ouest, la SanagaMaritime, Dibombari et le Nyong-et-Kéllé. Le bassin de production du département du Sud, où est implantée la SOCAPALM à Kienké, n’a pas fait l'objet d'un travail de terrain car les petits planteurs y sont peu nombreux et la direction de la SOCAPALM a été rencontrée à Douala.

Ce rapport est structuré en quatre parties : la première décrit les pratiques traditionnelles de l'exploitation ancestrale du palmier à huile au Cameroun en s'appuyant sur l'exemple du pays Bassa'a ; la deuxième présente la transition entre cette exploitation traditionnelle et l'exploitation du palmier à huile en plantations pendant la période coloniale; la troisième partie s'intéresse au développement de la filière au lendemain de la Deuxième Guerre mondiale puis après l'Indépendance. Enfin, l'analyse s'achève par les recompositions récentes de la filière, en cours depuis le début des années 1990, suite au désengagement de l'État. 


\section{Exploitation ancestrale du palmier à huile au Cameroun}

\subsection{Le palmier à huile, une culture ancrée dans la tradition}

Au Cameroun, l'exploitation du palmier par les communautés locales constitue un héritage social et culturel, la principale source de corps gras alimentaire, et une importante source de revenus. Jusqu'à la période coloniale, le palmier n'est pas cultivé en plantation avec un dispositif, mais il est présent à faible densité dans les parcelles destinées principalement aux productions vivrières. L'exploitation de ces palmiers proche de la cueillette, s'effectue en permanence même pendant les périodes de jachères agricoles. Les palmiers sont épargnés lors des défriches-brûlis par un nettoyage au sol, évitant ainsi que le feu ne s'en approche trop. Il existait également des peuplements sub-spontanés de palmiers qui étaient exploités en cueillette de la même manière ; ces peuplements recolonisaient souvent des sites de villages abandonnés par des populations peu fixées (Hartley, 1952).

Lorsque les colons européens débarquent en Afrique en général et au Cameroun en particulier, ils décrivent une palmeraie "naturelle ", qui était en fait la conséquence de ces activités humaines. Ce qualificatif abusif de "naturelle " va rester dans la littérature. La récolte des régimes s'effectuait en grimpant au stipe des grands palmiers avec des ceintures spécialement confectionnées pour cette opération. L'extraction de l'huile se faisait par foulage au pied et lavage à l'eau. Cette exploitation traditionnelle joua un rôle capital dans l'économie du Cameroun de la fin du XIX ${ }^{e}$ siècle jusqu'au début de la deuxième décennie du $\mathrm{XX}^{\mathrm{e}}$ siècle. Cependant, malgré quelques divergences liées aux spécificités socioculturelles et économiques propres à chaque peuple de la zone élæicole, les méthodes d'exploitation du palmier à huile sont sensiblement les mêmes d'une région à l'autre. Pour tenter de les décrire, nous insisterons ici sur le pays Bassa'a ${ }^{1}$ qui est représentatif de cette exploitation élæicole ancestrale. Précisons que l'épithète ancestrale fait référence ici à cette agriculture proche de la cueillette, aux pratiques coutumières, à l'extraction traditionnelle par foulage au pied observées dans la région. Certaines de ces pratiques subsistent de nos jours.

\subsection{Les pratiques agricoles ancestrales dans les palmeraies " naturelles "}

Les palmiers de la palmeraie «naturelle» font d'abord l'objet d'une sélection massale pied par pied par les populations qui éliminent les palmiers improductifs et diffusent par repiquage de plantules ou semis de graines à la volée, ceux dont les qualités sont appréciées (Hartley , 1952). Les palmiers à éliminer sont rapidement abattus et destinés à la production de vin de palme et d'autres sous-produits. Les palmiers dont la production est appréciée sont conservés dans les parcelles en rotation jachère - cultures vivrières. Lorsque la densité des plants est trop élevée, certains sont coupés pour laisser plus d'espace à ceux qui semblent plus aptes à produire. Pour pouvoir récolter régulièrement les palmiers productifs, des sentiers sont entretenus notamment pendant les périodes où les parcelles sont en jachère. Ils sont protégés du feu lors du brûlis qui précède la remise en culture de la parcelle. Lors de la récolte, les palmes gênantes sont coupées, ce qui entretient indirectement les couronnes, tandis qu'un rapide défrichage au sol permet de ramasser les fruits détachés. Près des cases, l'entretien des palmiers est le plus soigné. L'attention du chef d'exploitation diminue au fur et à mesure qu'on s'éloigne des

1 Le pays Bassa’a représente l'ensemble formé par les départements de la Sanaga-Maritime et du Nyong-et-Kéllé actuels. Jusqu'en 1958, ces deux départements formaient la Sanaga-Maritime. 
habitations en raison des contraintes de distance et de temps. La proximité avec le village facilite ainsi le suivi qui peut être confié aux femmes et aux enfants tandis que la récolte et l'entretien des sentiers menant aux palmiers éloignés, difficilement accessibles à ces deux groupes de population, incombent presqu'exclusivement aux hommes adultes.

\subsection{L'obtention des produits du palmier à huile}

En milieu paysan, toutes les parties du palmier à huile sont valorisables. Cependant, trois produits principaux ont une importance soit alimentaire, l'huile de palme, soit sociale, le vin de palme, ou encore thérapeutique dans la pharmacopée traditionnelle, l'huile de palmiste. La production des huiles de palme et de palmiste contenues dans les fruits nécessite de récolter des régimes, puis d'extraire d'abord l'huile de palme de la pulpe, puis l'huile de palmiste contenue dans l'amande. La production de vin de palme nécessite de récupérer la sève élaborée qui fermente spontanément.

\subsubsection{La récolte des régimes}

Le palmier étant une plante à croissance indéfinie, plus il pousse en hauteur avec l'âge, et plus il devient difficile de repérer les régimes mûrs. Or, si un régime mûr n'est pas coupé lors d'un passage de récolte, au tour de récolte suivant il sera pourri. $\mathrm{La}$ présence de régimes mûrs sur les grands palmiers est révélée par la prolifération de certains rongeurs comme les rats palmistes et les mangoustes.

La fin de la saison sèche annonce également le début de la période de pointe de production du palmier à huile. À partir de décembre les villageois commencent à surveiller les palmiers. La présence de fruits mûrs sur le sol au pied des palmiers indique qu'un ou plusieurs régimes sont mûrs et prêts à être coupés.

La coupe des régimes de palme est confiée à de véritables professionnels, des grimpeurs localement appelés «nkotbanga " ${ }^{2}$ payés à la tâche, à des prix

2 Généralement, les grimpeurs professionnels travaillent mal. Ils se soucient peu de l'entretien du palmier. Seuls les régimes les intéressent car leur salaire est adossé au nombre de régimes récoltés. variables en fonction des périodes, des régions et de la hauteur des palmiers et donc aux risques pris par le grimpeur pour aller récolter de plus gros régimes dans les palmiers les plus hauts. Afin de couper les régimes, les récolteurs coupent les palmes gênantes mais ils doivent aussi élaguer les palmes sèches de la couronne pour garder une bonne réputation dans le village et donc des opportunités d'emplois. En fonction de la qualité du travail, du coût et de l'appartenance clanique ou lignagère, une certaine fidélisation se tisse entre les grimpeurs et les ayant droits des terres où poussent des palmiers. Toutefois, certains exploitants s'occupent euxmêmes de la coupe de leurs régimes et peuvent aussi être des grimpeurs professionnels.

Les principaux outils de travail sont une machette bien affûtée et un cerceau ellipsoïdal à grimper appelé localement «lemb» fabriqué soit avec des nervures de palmes soit en rotin (photo 1). Il s'agit en fait de deux cordes d'égale longueur reliées aux deux bouts par des nouds dont l'un est fixe et l'autre régulièrement attaché et détaché à chaque montée et à chaque descente. Lorsque ce sont les nervures de palme qui sont utilisées, la partie sur laquelle le grimpeur appuie les reins est aplatie pour améliorer le confort, alors que dans le cas

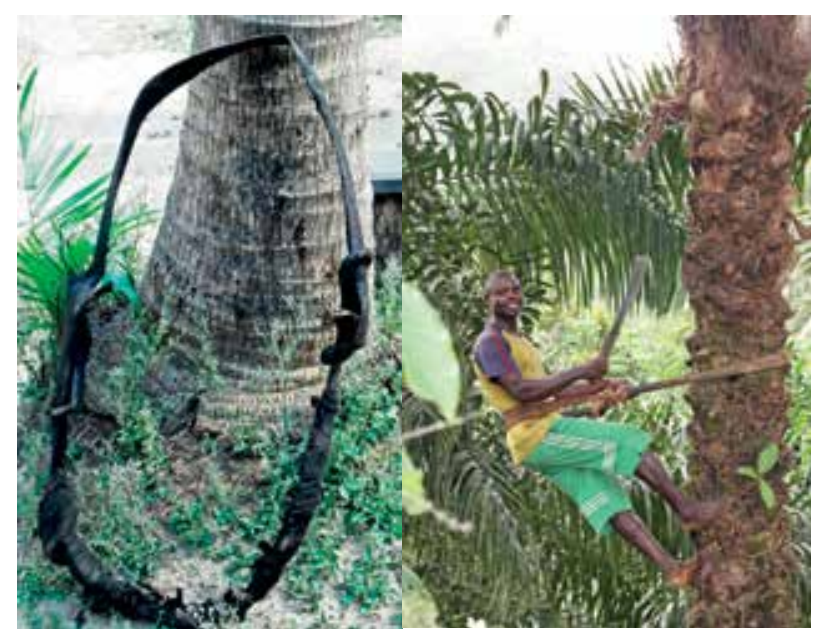

Photo 1 : Le lemb, cerceau ellipsoïdal à grimper Ce cerceau fait en rotin a plus de 10 ans d'âge. Remarquez les deux nœuds à gauche et à droite du lemb. Lors de la cueillette, la partie haute s'accroche au palmier tandis que la partie au sol s'appuie autour des reins du grimpeur. Elle est entourée de vieux tissus pour améliorer le confort.

Clichés : Ndjogui, février 2014 ; Levang, février 2011 
de l'utilisation du rotin, cette partie est tressée afin d'adoucir la rugosité du rotin. Le lemb est généralement conservé sur la claie au-dessus du feu pour le durcir davantage. Un lemb dure entre un an et un an et demi selon que l'on utilise le rotin ou la nervure de palme.

Après avoir préalablement nettoyé les alentours du palmier, le grimpeur enfile le lemb autour de ses reins, les mains tenant les deux nœuds $\mathrm{du}$ lemb, et pose alternativement les pieds sur le stipe du palmier. Il se déplace en posant ses pieds sur les encoches préalablement entaillées sur le stipe, le «tronc » du palmier. En effet, l'une des caractéristiques du matériel végétal local ou " sauvage " est la chute précoce des chicots des palmes par rapport au matériel sélectionné, laissant ainsi le stipe lisse. La ceinture se déplace au même rythme que les pieds se posent sur les entailles. Pendant la montée, la machette est maintenue coincée entre la joue et l'épaule. Arrivé à la couronne, le grimpeur coupe les palmes en-dessous des régimes mûrs pour pouvoir couper le pétiole du régime. Après la coupe, il élague le palmier pour faciliter la prochaine coupe. Ce travail terminé, le grimpeur descend du palmier, détache la ceinture et entasse tous les déchets hors du rond nettoyé pour ramasser les fruits détachés. Les régimes sont ensuite transportés vers le lieu d'extraction de l'huile de palme.

\subsubsection{La production $d$ 'huile de palme}

L'extraction d'huile de palme contenue dans la pulpe des fruits s'effectue en plusieurs phases: l'égrappage des régimes, la cuisson des fruits, le pilage, la trituration et la clarification. Avant le processus de transformation proprement dit, les régimes de palme suivent un prétraitement consistant en l'égrappage des fruits. Après la coupe, les régimes sont laissés en tas pendant un à deux jours et parfois une semaine pour faciliter l'égrappage. Le procédé d'égrappage fait intervenir deux options selon le degré de maturité des régimes. Lorsque les régimes sont bien mûrs et tendres, la procédure consiste à frapper le régime avec un bâton ou le revers d'une machette ou d'une hache. Par contre, lorsqu'il n'est pas encore bien mûr, il est d'abord nécessaire de séparer les épillets de la rafle avant de procéder à l'égrappage manuel des épillets. Le travail d'égrappage est souvent confié aux enfants et aux femmes.

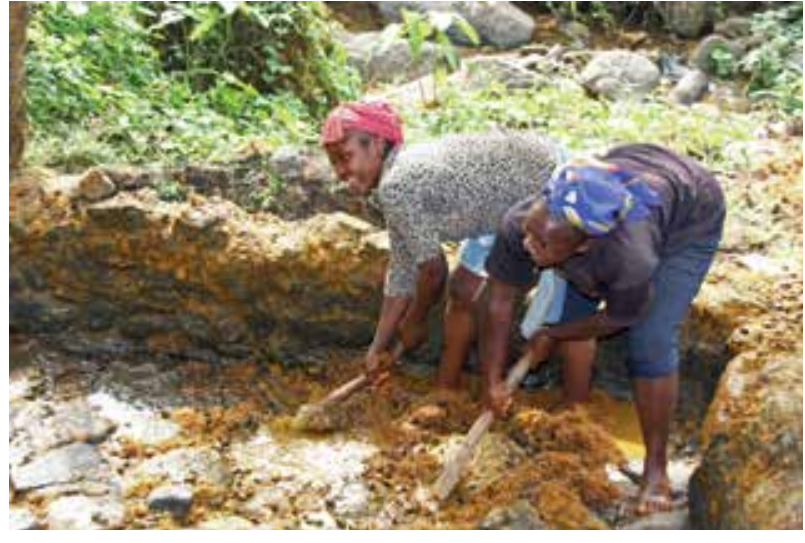

\section{Photo 2 : Lieu d'extraction traditionnelle de l'huile} de palme à Fontem (Sud-Ouest)

Les pierres délimitent l'aire d'extraction et permettent d'emprisonner l'eau. Le centre de la photo est le lieu de foulage proprement dit. Une partie en creux permet de collecter le jus brut crémeux contenant l'huile de palme.

Cliché : Levang, février 2011

Après l'égrappage, commence la cuisson. Les fruits sont versés dans une grande marmite avec de l'eau. La nature des contenants a varié avec le temps, passant de l'utilisation des marmites en terre cuite à celle des marmites en aluminium, puis des fûts métalliques de 220 litres. La cuisson d'un fût de fruits peut durer quatre à sept heures en fonction de la puissance du feu. Pour accélérer la cuisson, un « couvercle végétal » de feuilles de macabo est posé au-dessus des fruits. Une odeur particulière indique que les fruits sont cuits à point. Une fois cuits, les fruits sont pilés dans un mortier puis la pulpe est séparée des noix par foulage au pied dans une cuvette façonnée dans le sol, durcie à l'argile ou avec du ciment. Ce lieu de foulage au pied s'appelle wa'a en langue Bassa'a (photo 2). C'est un lieu spécialement aménagé sur la berge d'un cours d'eau pour l'extraction traditionnelle de l'huile de palme.

L'opération de foulage au pied requiert deux personnes au moins, en général des femmes. Pendant que l'une foule les fruits avec ses pieds, l'autre verse de l'eau de temps en temps dans le $w a^{\prime} a$ en suivant les instructions de la première ${ }^{3}$. Au fur et à mesure que le foulage avance, on débarrasse progressivement le $w a^{\prime} a$ des fibres

3 Dans la variante du Nyong-et-Kéllé, les fruits cuits sont directement versés dans le wa’a et ne sont pas préalablement pilés. 
appelés localement bikakang, et on récupère les noix de palmiste afin d'éviter sa saturation. L'eau versée dans le $w a$ 'a s'accumule progressivement dans la partie plus en creux tandis que l'huile, le jus brut ou yjoñ en Bassa'a, surnage. Au final, on obtient une mare d'eau jaunâtre constituée de deux phases. En-dessous, il y a l'eau et les détritus tandis qu'au-dessus flotte le yjoñ, jus brut crémeux puisque l'huile de palme est concrète (solide à température ambiante généralement autour de $20^{\circ} \mathrm{C}$ ). C'est cette dernière partie que l'on recueille à l'aide d'une calebasse et que l'on verse dans un récipient prévu à cet effet. Après le foulage, le $w a^{\prime} a$ est nettoyé et lavé, et la crème est transportée au lieu de cuisson qui est aménagé à côté du lieu de foulage. Cependant, le foulage peut aussi se faire à la main et à la maison lorsque les fruits sont peu nombreux. Dans ce cas, le wa'a est remplacé par une simple bassine. Il faut alors s'assurer de disposer de suffisamment d'eau.

Ensuite, débute l'opération de " clarification » du jus brut qui dure moins de deux heures. Le $\eta j o \tilde{n}$ est posé sur le feu et cuit afin de séparer l'huile des impuretés fines et d'évaporer l'excès d'eau. La clarification est achevée lorsque la mousse formée à la bouche de la marmite ou du fût devient noire, et que l'odeur caractéristique de l'huile de palme rouge commence à se dégager. Il est alors nécessaire d'enlever les petits récipients du feu, ou bien de réduire l'intensité du feu dans le cas des fûts. Quel que soit le contenant, le produit est laissé au repos jusqu'à refroidissement et décantation complète. Ensuite, à l'aide d'une calebasse, l'huile qui surnage au-dessus des détritus est recueillie et versée dans un autre récipient pour continuer la décantation. C'est de là que l'huile sera transvasée dans les bidons ou d'autres contenants avant la consommation ou la commercialisation. L'huile de palme rouge et la crème à l'état brut rentrent dans la préparation de presque tous les mets traditionnels Bassa'a.

\subsubsection{La production de I'huile de palmiste}

Les noix de palmiste sont récupérées dans le $w a^{\prime} a$, mais aussi ramassées au champ, là où des régimes ont pourri. Après leur collecte, les noix sont séchées au soleil ou déposées sur une claie au-dessus du foyer afin de sécher l'amande qui se décolle alors facilement de la coque. Ceci facilite ensuite le concassage qui consiste à séparer l'amande de la coque. Ensuite, l'amande est à nouveau séchée au soleil ou sur la claie. L'objectif est de diminuer le taux d'humidité des noix. Une à deux semaines après, les amandes sont versées dans une marmite posée sur le feu pour y être chauffées afin que l'huile exsude des amandes. De temps en temps, on rajoute un peu d'eau à la marmite. Pour améliorer le parfum, des feuilles de safoutier sont parfois ajoutées dans la marmite. Au bout d'une trentaine de minutes, on commence à entendre des bruits d'éclatement des amandes accompagnés de fumées asphyxiantes qui signalent la fin du chauffage des amandes. La marmite est alors enlevée du feu et posée au soleil pour décantation. On obtient finalement une huile noire, localement appelée lañ, que l'on sépare des amandes résiduelles également devenues noires. Cette huile joue un rôle important dans la pharmacopée et la cosmétique traditionnelle, et tout particulièrement dans l'entretien de la peau des nouveaux nés. Encore de nos jours, la majorité des femmes Bassa'a qui viennent d'accoucher utilisent le lañ pour oindre la peau de leurs bébés et pour leur propre traitement postpartum. Certaines préparations pharmaceutiques demandent de ne pas surchauffer les amandes, ce qui diminue le rendement d'extraction, mais permet d'obtenir une huile blanche qui a d'autres propriétés que l'huile noire.

\subsubsection{La production du vin de palme}

Le vin de palme ou matañgo joue un rôle fondamental dans la société traditionnelle. Aujourd'hui il constitue en plus une importante source de revenus pour de nombreux ménages ruraux et surtout péri-urbains. En effet, la consommation du vin de palme est également en augmentation dans les zones urbaines, où son prix de vente très attractif lui permet de concurrencer les produits des brasseries et les spiritueux présents sur le marché.

Il existe deux principales modalités d'extraction du vin de palme. La première est la récolte aérienne, plus répandue dans le groupe ethnico-linguistique Fang-Béti des régions Centre, Sud, et Est du Cameroun. Cette technique non létale consiste à recueillir la sève élaborée du palmier s'écoulant des pédoncules des inflorescences par une incision. La seconde technique de récolte consiste à extraire la sève du palmier à partir du bourgeon apical du palmier. Cette technique est létale puisqu'elle 


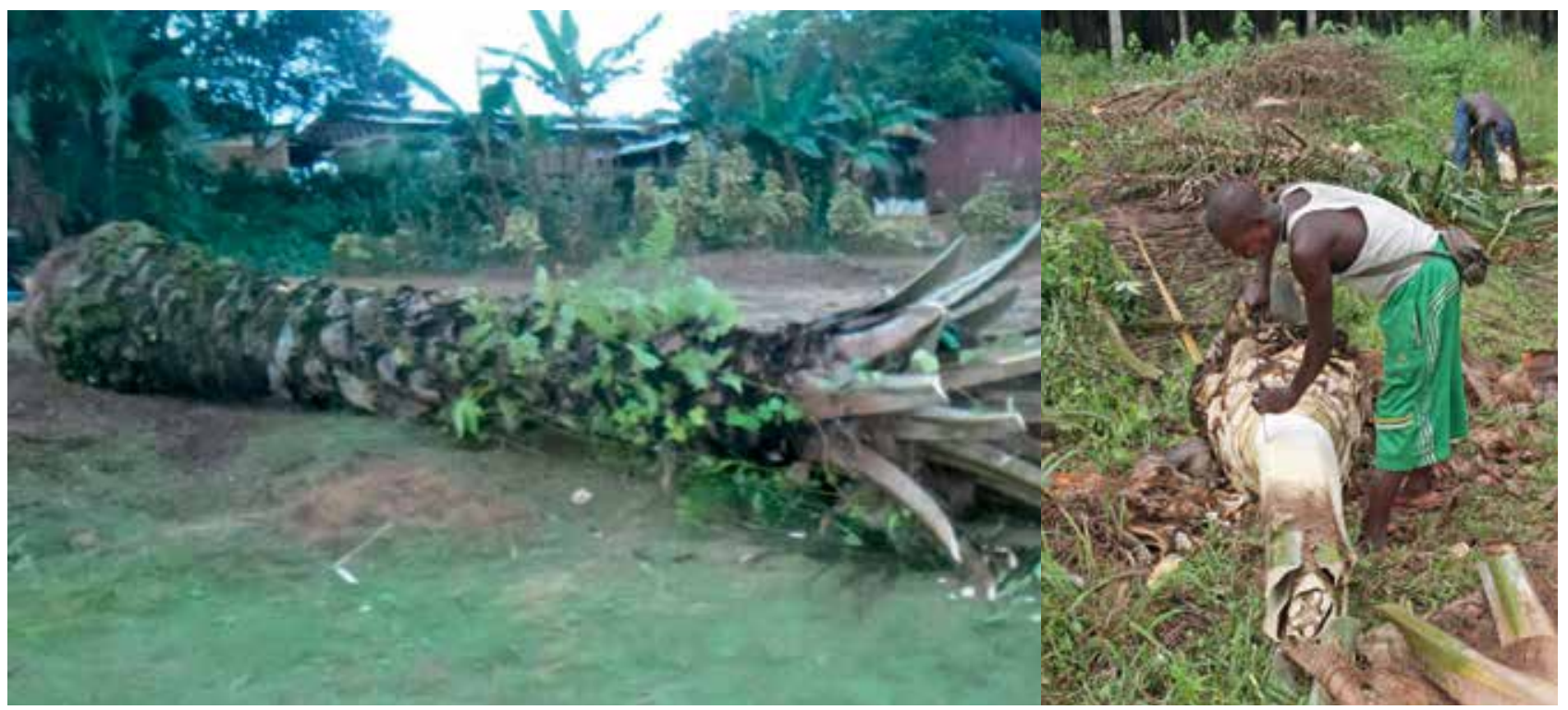

\section{Photo 3 : Un palmier déterré puis abattu en coupant les racines}

La couronne sera par la suite débarrassée de ses palmes comme on le voit sur la photo 4.

Clichés Ndjogui, juillet 2013 ; Levang, février 2013

nécessite l'abattage ou le déracinement de l'arbre selon l'âge. Les palmiers jeunes sont habituellement déterrés, alors que les vieux sont abattus (photo 3). C'est cette dernière technique qui offre les meilleurs rendements et est la plus répandue en pays Bassa'a ; nous la décrivons ici en détail.

Après le déracinement ou l'abattage du palmier, l'exploitant doit couper toutes les palmes de la couronne afin de mettre à nu le bourgeon apical, localement appelé mom en langue Bassa'a. Ce dernier est alors entouré d'un entonnoir fabriqué à base de fibres provenant des nervures appelées ngañgabi, au bout duquel on place un récipient qui va recueillir la sève qui s'écoule et fermente spontanément pour devenir le vin de palme (photo 4). Le lendemain, le récolteur revient détacher l'entonnoir et récupérer le vin. Avec une machette bien affutée et généralement réservée à ce travail, il coupe une fine tranche du mom qui a cicatrisé pour raviver la plaie et permettre à la sève de continuer à s'écouler. Cette opération est renouvelée matin et soir produisant ainsi un vin du matin et un vin du soir. Un palmier "saigné " produit pendant environ 1 mois entre 1 et 5 litres de vin par saignée selon le volume du stipe, qui dépend lui-même de l'âge du palmier, des conditions de culture et de la nature du matériel végétal.

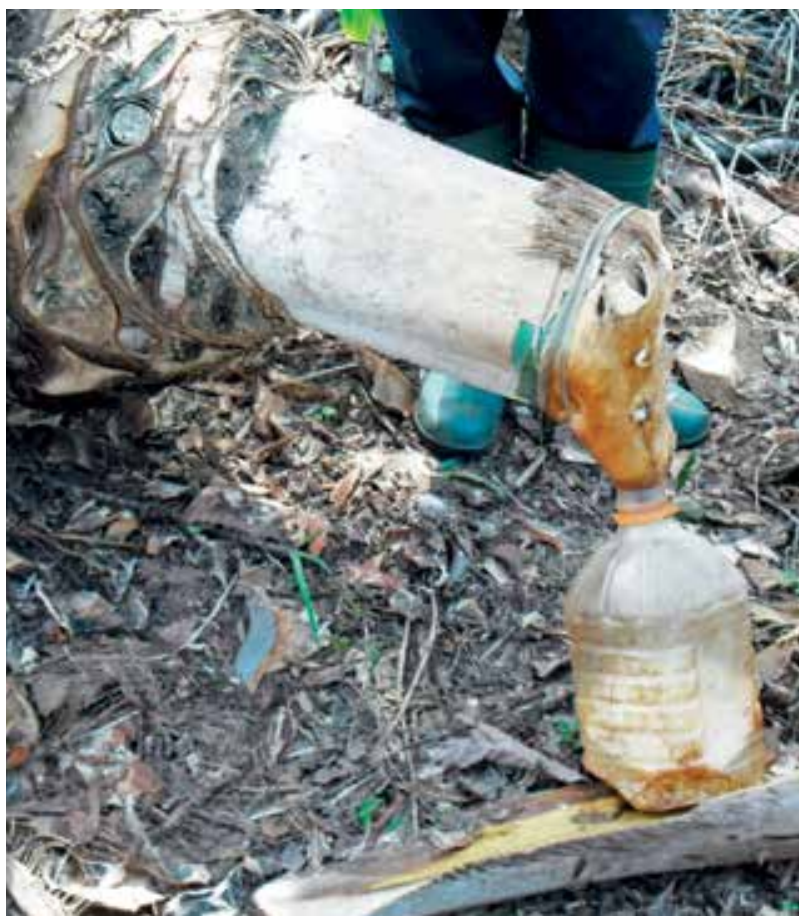

\section{Photo 4: La sève qui s'écoule du bourgeon apical fermente spontanément en se transformant en vin de palme}

L'extrémité du bourgeon apical (mom) est couverte d'un entonnoir fait de nervures appelées ngañgabi qu'on aperçoit au-dessus du bidon recueillant la sève.

Cliché Ndjogui, juillet 2013 
Le vin ainsi produit est destiné à

l'autoconsommation ou à la vente, parfois les deux. Autrefois, le vin n'était pas vendu mais était cordialement partagé entre amis ou utilisé pour diverses manifestations socioculturelles. La vente du vin de palme est un phénomène relativement récent et date de la fin du XIX ${ }^{\mathrm{e}}$ siècle avec l'arrivée des Européens et la monétarisation de l'économie rurale. Avant cette période, le vin de palme était surtout échangé dans un système de troc.

Dans les années 1970, un litre de vin de palme coûtait entre 15 et 18 francs CFA. De nos jours, un litre est vendu entre 100 et 250 francs CFA, respectivement au village et en ville. Le commerce du vin de palme est surtout florissant dans les zones périurbaines des grands centres urbains comme Douala et Yaoundé où il existe des bars spécialisés fréquentés par de nombreux consommateurs. Compte tenu du fait que la fermentation de la sève n'est pas interrompue, le vin devient de moins en moins sucré et de plus en plus acide au fil du temps. Ceci explique la nécessaire proximité entre les zones de production de vin destiné à la vente et les grands centres de consommation.

C'est surtout sur le plan socioculturel que le vin de palme garde toute son importance et toute sa symbolique. Sa valeur culturelle résiste à la " modernité » et à la monétarisation en milieu rural loin des grandes villes. Ainsi le vin de palme est indispensable à la dot de la mariée. Il ne tarit pas dans la gourde du $m b o m b o k^{4}$ qui l'utilise soit pour se raviver soit pour bénir une personne en association avec une noix de colatier ou pour lui marquer son affection. Étymologiquement, le vin de palme appelé localement moog $^{5}$ ma maen $^{6}$ est un élément de bénédiction. C'est pourquoi il constitue avec la cola l'un des éléments que les mbombok utilisent pour bénir une personne. C'est également la boisson magique des sociétés secrètes comme les ngué et les um.

Enfin, le vin de palme joue également un rôle de premier plan dans les rapports intergénérationnels. Ainsi, un cadet a le devoir de présenter le

4 Patriarche et chef religieux en pays Bassa’a.

5 Dans le département du Nyong-et-Kéllé, on dit plutôt maoog.

6 Maén signifie les palmiers. Au singulier, on dit lién. Dans le langage courant, le vin de palme est appelé matango plus particulièrement en milieu urbain. premier vin de son palmier appelé liyal à son aîné, accompagné d'un cadeau spécial. C'est ce dernier qui invite les convives à le boire car le vin de palme ne se boit pas en cercle fermé ni exclusivement entre les membres d'une même famille (Tjééga, 1974). C'est toujours l'aîné qui donne l'ordre à son cadet d'initier la distribution du vin. Le vin de palme joue ainsi un rôle important dans la reproduction de la hiérarchie sociale et véhicule de nombreuses valeurs telles le respect, le partage et l'hospitalité. Un étranger de passage s'arrête lorsqu'il trouve les gens en train de boire du vin de palme et reçoit successivement trois gobelets. De la sorte, l'étranger établit le contact avec les villageois avant de continuer sa route.

Certains artisans spécialisés stockent dans des fûts le vin de palme de plusieurs palmiers abattus jusqu'à ce que la fermentation cesse par épuisement des sucres contenus dans la sève. Ils en extraient ensuite de l'eau de vie ou alcool de palme par distillation appelé ha'a en bassa'a ou odontol en langage trivial.

\subsection{Une forme d'exploitation qui résiste à l'épreuve du temps}

Le palmier à huile est une plante caractéristique du paysage côtier du pourtour du golfe de Guinée en général et du pays Bassa'a de manière spécifique. Il est la marque de la présence humaine dans le paysage. Sa multiple vocation alimentaire, sociale et culturelle explique son exploitation ancestrale par les communautés locales. Jusqu'à la fin du XIX siècle, le palmier à huile n'est pas planté sauf dans des cas rares comme par exemple le marquage foncier. Son exploitation ne consiste qu'en une simple cueillette des sujets qui poussent spontanément dans la nature et particulièrement dans les jachères agricoles. De nos jours, les systèmes d'exploitation traditionnelle restent proches des techniques et usages ancestraux. Malgré les méthodes et les techniques d'extraction qui ont été améliorées par le développement de la transformation artisanale, les produits $\mathrm{du}$ palmier élaborés de manière artisanale gardent leur importance et leur prestige. Il en est ainsi de l'huile de palme et de l'huile de palmiste artisanales qui sont davantage appréciées pour la cuisine et la pharmacopée traditionnelles. De par leur valeur marchande et leur usage industriel potentiel, ces produits vont jouer un rôle déterminant dans les contacts entre les Occidentaux et les autochtones lors de la colonisation. 


\section{Les prémisses du developpement élæicole}

\subsection{Les produits du palmier à la veille de la colonisation européenne}

À la veille de la colonisation, les produits du palmier, notamment l'huile de palme et les noix de palmiste, font partie des principaux objets d'échange sur la côte camerounaise. Elles permettent des échanges non seulement au sein des régions productrices, mais aussi avec l'extérieur. Au début du XIX ${ }^{\mathrm{e}}$ siècle, de nombreux auteurs (Robertson, 1819 ; Bidjeck, 2007 ; Barbier, 1971 cité par Tchiabou Tchinda, 2007) signalent que 50 à 60 tonnes d'huile de palme sont exportées en 1810. En 1856, le principal trafic est celui de l'huile de palme, dont on exporte plus de $2000 \mathrm{t}$ chaque année contre $200 \mathrm{t}$ en 1860 , quand la noix de palmiste supplante l'huile de palme dans les échanges du Cameroun avec l'extérieur (Bauchaud, 1952). À la fin du XIX ${ }^{\mathrm{e}}$ siècle, une tonne d'ivoire (équivalant à 240 livres sterling) plus 10 tonnes de sel s'échangeaient contre $20 \mathrm{t}$ d'huile de palme sur la côte camerounaise (Adams, 1823). Bref, la commercialisation des produits du palmier à huile, en l'occurrence l'huile de palme et les noix de palmiste, était prospère à la veille de la colonisation européenne. Cependant, ces produits étaient exclusivement issus de la récolte de la "palmeraie naturelle " constitués d'un matériel végétal sauvage à faible rendement en régimes et à très faible teneur en huile dans les fruits. Les méthodes et les techniques d'extraction traditionnelle étaient très coûteuses en main d'œuvre. C'est dans ce contexte que le protectorat allemand intervient à la fin du XIX ${ }^{\mathrm{e}}$ siècle. Il ouvre une nouvelle ère à l'exploitation du palmier à huile au Cameroun avec la création des premières plantations coloniales.

\subsection{Les premières plantations de palmier à huile au Cameroun}

La culture du palmier à huile en plantation naît avec la création des premières palmeraies allemandes sur les pentes du Mont Cameroun.

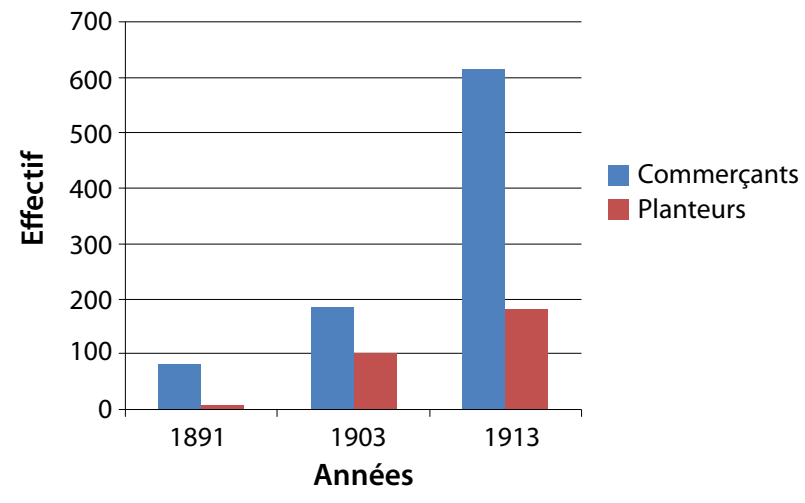

Figure 5 : Les flux migratoires allemands au Cameroun (1891-1913)

Source : Etoga, 1971

Au tout début de l'occupation allemande, c'est le commerce qui présente un intérêt pour la métropole (fig. 5), puis la création de plantations est envisagée d'abord dans l'actuelle région du Sud-Ouest, en restant une préoccupation secondaire. En effet, c'est le cacao qui retient d'abord l'attention des Allemands qui créent la West Afrikanische Pflanzungs Gesellschaft en 1897, considérée à l'époque comme la plus vaste cacaoyère du monde avec 4000 ha (Etoga, 1971).

Jusqu'en 1888, les noix de palmiste qui ne sont pas utilisées pour extraire de manière traditionnelle de l'huile de palmiste sont habituellement jetées par les paysans des flancs du Mont Cameroun, un gaspillage qui consterne les Allemands. Le Docteur Haake invente alors une presse artisanale permettant d'extraire l'huile de palmiste des amandes (Etoga, 1971). Les premières plantations de palmier à huile voient le jour à partir de 1898 avec la création de la Debundcha Palm Company (Debundcha Pflanzung) sur le versant ouest du Mont Cameroun. Cependant, les activités de cette plantation ne deviennent effectives qu'en 1904 (Ngoh, 1996 cité par Ayuketang, 2010). Plus tard, en 1907, les Allemands créent la première plantation « industrielle » de palmier à huile au sud 


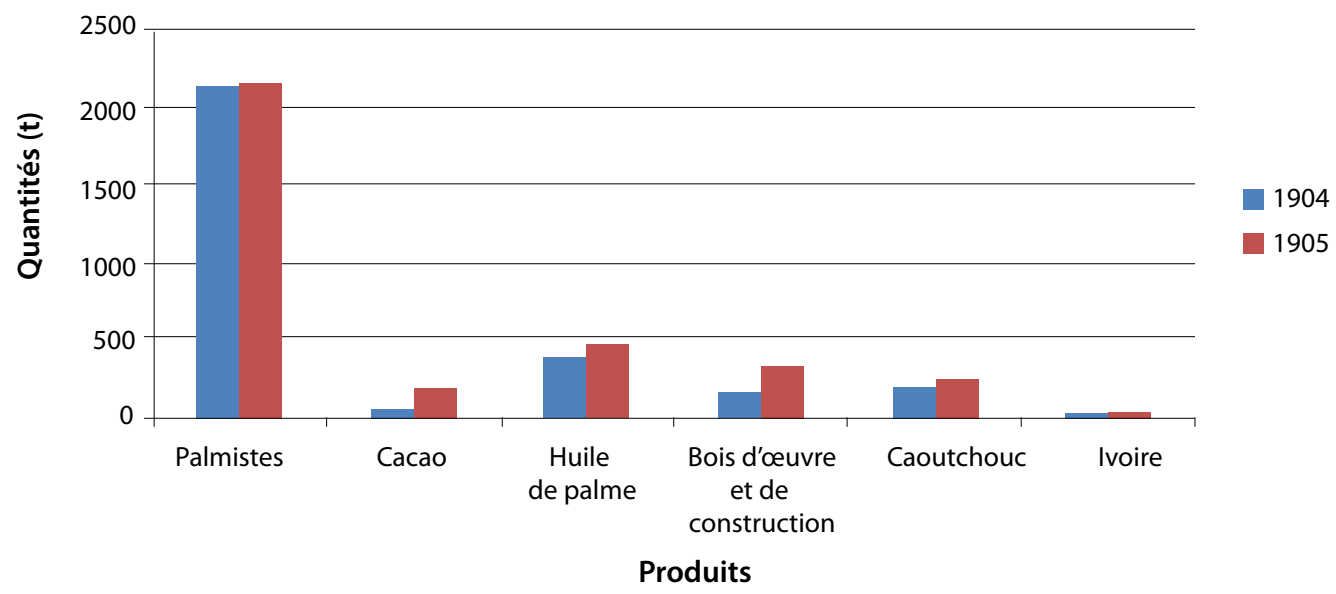

Figure 6 : Exportations du Cameroun (1904-1905)

Source : Etoga, 1971

d'Edéa (Etoga, 1971), aujourd'hui connue sous le nom de Société des Palmeraies de la Ferme Suisse. Au début du $\mathrm{XX}^{\mathrm{e}}$ siècle, les productions d'huile de palme et de palmiste dépassent en tonnages celle de cacao et de caoutchouc (fig. 6).

Cette réussite incite les Allemands à développer davantage de palmeraies. À la fin du protectorat allemand notamment entre 1912 et 1913 , ils plantent 5044 ha de palmiers à huile sur les pentes du Mont Cameroun. Il s'agit probablement de la variété lisombe ${ }^{7}$ qui est la meilleure espèce de palmier connue de l'époque qui aurait permis de réaliser un bénéfice de 2014 Deutsche Marks / ha/an. Notons qu'à la même période il existe une petite savonnerie à Douala dont la production est consommée localement (Etoga, 1971).

Parallèlement à la création des premières plantations industrielles, quelques peuplements de palmiers "naturels » sont loués dès 1914 aux communautés indigènes par les propriétaires de grandes plantations (Jannot, 2002 cité par Fevre, 2002), traduisant ainsi le besoin de régimes pour produire davantage d'huile. Malheureusement, le plan de développement du palmier qui devait commencer en 1912 ne sera pas mis en œuvre à cause du déclenchement de la Première Guerre mondiale.

A l'inverse de la situation actuelle, les tonnages de palmistes exportés sont nettement supérieurs aux

7 Certains auteurs écrivent Lisonde. Lire Etoga, 1971. Sur les chemins du développement. Essai des faits économiques du Cameroun, p. 163. tonnages d'huile de palme exportés. Même si le matériel de type dura de l'époque avait une pulpe très fine et une grosse amande comparativement au matériel actuel, cette inversion de proportion est trop importante pour ne se justifier que par le matériel végétal exploité, comme nous l'expliquons dans le point 3.3.2.

\subsection{La Guerre de 1914-1918 et le début des plantations managériales des colons}

La Première Guerre mondiale constitue une rupture majeure dans le développement économique et social du Cameroun en général et celui du palmier à huile en particulier. La première conséquence de cette guerre est la scission du territoire en deux administrations distinctes dès 1916. Elle implique également la dispersion des biens allemands tombés entre les mains des vainqueurs que sont les Anglais et les Français.

\subsubsection{La situation au Cameroun sous administration anglaise}

Après la défaite allemande en 1916, les communautés locales, notamment les Bakwéri spoliés de leurs terres par les Allemands, espèrent se les réapproprier. Mais certains hommes politiques britanniques comme Moorehouse MC s'opposent à cette idée. Pour lui, "It will be impractical to split up the plantations into small plots for native owners. Without capital, it will mean that the buildings and 
machinery would fall into ruins entailing enormous loss, and without the necessary experience, diseases will soon spread» (Ayuketang, 2010). Aux yeux des administrateurs coloniaux, seuls les Anglais et les Américains peuvent disposer des capitaux et de l'expérience à même de garantir une bonne gestion de ces plantations et de préserver la qualité de leur exploitation. En 1920, le traité de Versailles entre en vigueur et consacre la naissance de la Société des Nations. La partie camerounaise sous administration anglaise intègre officiellement le Nigéria. Puis, sur la base de l'article 279 (02) du traité de Versailles qui laisse le choix aux alliés soit de s'approprier les anciennes plantations allemandes, soit de les rétrocéder aux populations autochtones (Le Vine, 1964), les plantations allemandes sont mises aux enchères. En 1922, une première tentative de vente aux enchères a lieu et exclut les Allemands des transactions. Une faible partie des plantations trouve des acheteurs pour des raisons variées telles que l'incertitude du devenir du nouveau mandat, la difficulté de réunir le capital nécessaire à la gestion des palmeraies, la mauvaise lisibilité des titres fonciers ou même l'éruption volcanique de $1922^{8}$ (Limoigne, 2010). Les Allemands qui souhaitent participer à ces opérations sont obligés d'utiliser des prête-noms ou des hommes de paille qui négocient à leur place (Courade, 1980). Les autorités britanniques proposent une nouvelle vente en 1924, cette fois en levant l'exclusion des Allemands dans les transactions. Ces derniers rachètent la quasi-totalité des lots (Courade, 1979 ; Molua, 1985). En 1926, sur 219 Européens possédant des palmeraies sur les flancs du Mont Cameroun, 136 sont Allemands soit $62,10 \%$ de l'effectif total (Gardinier, 1967).

Le Cameroun sous mandat britannique est le domaine des grandes plantations héritées du protectorat allemand. Le cas de la PAMOL est assez illustratif. En effet, pendant la vente aux

8 En 1922, après treize ans de repos, le volcan entre à nouveau en activité. L'éruption dure sept mois, du 3 février au 24 août, et deux foyers d'éruption apparaissent, l'un au sommet du volcan et l'autre sur le flanc sud-ouest. Ce dernier produit un fleuve de lave pahoehoe qui se dirige vers Bibundi, détruisant sur son passage un pont sur la route de Bibundi à Victoria (actuel Limbe), des plantations de cacaoyers et d'hévéas, une voie ferrée d'exploitation et deux maisons de travailleurs, pour se jeter dans la mer. De nouvelles coulées de laves sont émises au mois de mai et atteignent à nouveau l'océan. Plus de cent personnes périssent lors de cette éruption. » (Lemoigne, 2010). enchères des biens allemands, quatre plantations sont achetées par les Anglais pour le groupe Unilever. Ces plantations incluent Bwenga et Bai pour l'hévéa, Lobe et Ndian pour le palmier à huile. En dehors de ces plantations, le groupe obtient une concession de 10000 ha de forêts secondaires et primaires pour la création de la «Pamol Plantations Limited " avec pour principal objectif la culture du palmier à huile. Le domaine de Lobé avait d'abord appartenu à la firme allemande Deutsche Westafrikanische Handelsgesellschaft (DWH) avant la Première Guerre mondiale. Il est racheté par un particulier en 1922 et revendu à la DWH en 1927. En 1932, le domaine passe aux mains de 1'United Africa Company (UAC) dirigée par les frères Lever, la plus puissante compagnie de commerce du Nigéria. Cette dernière ne le met en valeur que dans les années 1950. La période d'entre-deux guerres voit la mise en exploitation de la plantation de Ndian. Entre 1928 et 1933, 6459 acres soit 2 613,86 ha de forêts sont défrichés et plantés en palmiers à huile et en 1935, une huilerie d'une capacité de 1000 t d'huile par jour est mise en place. En 1938, la plantation de Ndian est présentée comme la plus moderne du Cameroun sous tutelle anglaise.

A la veille du second conflit mondial en 1939, toutes les propriétés allemandes ainsi que les circuits de commerce du Cameroun sous mandat britannique sont entre les mains des compagnies allemandes ou des commerçants allemands installés à titre privé (Etoga, 1971). Cependant, dès les premiers bruits de la guerre, les biens allemands, à l'instar des plantations, sont de nouveau confisqués par les Anglais.

À la fin du conflit en 1945, le Gouvernement du Nigéria sous administration britannique décide d'enlever aux acquéreurs privés la possibilité de racheter les propriétés de l'ennemi, et choisit d'en conserver la gestion au bénéfice des populations $\mathrm{du}$ Cameroun occidental. Cette politique est fidèle aux directives de l'Organisation des Nations Unies (ONU) concernant les territoires désormais placés sous sa responsabilité. L'ensemble des biens saisis y compris les plantations est confié au Gouverneur du Nigéria qui crée une société d'État chargée de les gérer. C'est ainsi que par une ordonnance de 1946, la Cameroons Development Corporation Ltd (CDC) est créée comme une régie du gouvernement camerounais sous tutelle anglaise. La nouvelle compagnie entre en activité 
en 1947. À cette date, l'ensemble de ses plantations atteignent 20000 ha (Limoigne, 2010). Les cultures développées incluent le bananier, le théier, l'hévéa et le palmier à huile. Entre 1946 et 1947, la CDC met sur pied le premier programme de plantations villageoises dans cette partie du pays. Malheureusement, ce projet est boycotté par les autochtones Bakweri qui revendiquent la rétrocession de leurs terres ancestrales confisquées par les Allemands depuis la fin du XIX ${ }^{\mathrm{e}}$ siècle.

\subsubsection{La situation dans le Cameroun sous administration française}

Dans le Cameroun sous administration française, une ordonnance du Tribunal de Douala du 16 novembre 1916 place tous les biens allemands sous séquestre et confie leur gestion à un mandataire de justice avec un droit de préemption reconnu au mandataire (Etoga, 1971). L'essentiel des anciennes plantations allemandes est acheté par les Français. Dès 1920, l'administration coloniale s'attaque d'abord au problème foncier. Elle abandonne le régime des grandes concessions en vigueur pendant la période allemande et opte pour les concessions moyennes. Elle prend également des mesures pour la protection des droits des autochtones et crée des réserves foncières pour le développement des cultures vivrières au bénéfice des autochtones. L'objectif est de soustraire certaines portions du territoire à la colonisation européenne (Etoga, 1971) afin que ces espaces soient à nouveau gérés par les populations locales.

La liquidation des biens allemands ne prend fin qu'en 1925, période pendant laquelle le palmiste et l'huile de palme sont les principaux produits d'exportation du Cameroun sous mandat. Les palmistes supplantent l'huile de palme dans les exportations des produits du palmier depuis une vingtaine d'années (figure 6). Ceci est lié à l'ouverture du pays aux échanges internes et surtout à la construction de la ligne de chemin de fer qui permet de collecter les palmistes provenant de l'intérieur du pays où on extrait par foulage au pied de l'huile rouge. Dans le Cameroun sous mandat français, l'implantation de palmeraies et d'huileries augmente également les exportations d'huile de palme qui atteignent 5420 t en 1928 et 8364 t en 1929. Lorsque la grande crise économique éclate aux États-Unis en 1929, elle entraîne la baisse des cours des

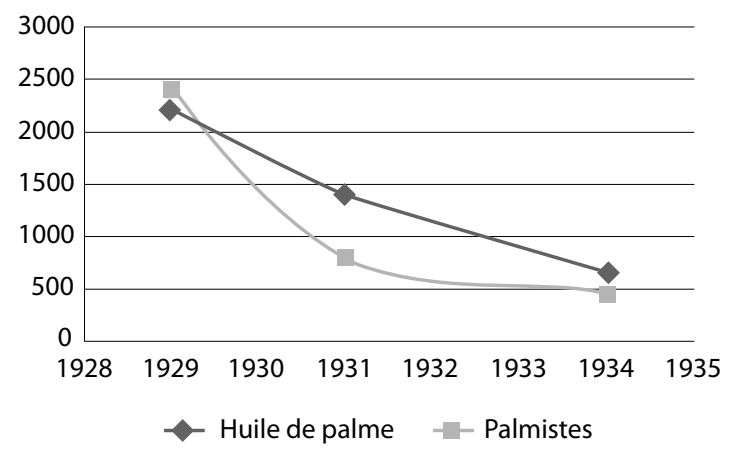

Figure 7 : Évolution des cours des produits du palmier à huile entre 1929 et 1934

Source : Etoga, 1971

produits du palmier. Le prix $\mathrm{FOB}^{9}$ à Douala de l'huile de palme et des palmistes connaît une chute vertigineuse (Fig. 7).

Les exportations d'huile de palme (fig. 8) connaissent également un fléchissement en 1931 passant de 8364 t en 1929 à 5869 en 1931 (Etoga, 1971). Notons qu'il est difficile d'estimer la production des produits du palmier à cette époque, car une partie des récoltes est consommée localement et échappe à la comptabilité. Seules les données relatives aux exportations sont disponibles et accessibles.

Pour éviter une implosion sociale liée au mécontentement des indigènes qui menacent d'abandonner l'exploitation du palmier à huile, les autorités coloniales adoptent des mesures d'urgence. Par l'arrêté de 1933, le tarif du transport ferroviaire est réduit de $50 \%$ pour les palmistes et de $75 \%$ pour l'huile de palme. Un autre arrêté suspend la perception de toutes les taxes liées à l'exportation de ces produits dès le $1^{\text {er }}$ janvier 1934. L'application de ces mesures permet le relèvement des prix FOB au port de Douala en janvier 1936. Les cours remontent à 680 francs pour les palmistes et à 1 170francs pour l'huile de palme (Etoga, 1971 ; Bidjeck, 2002).

Toutefois, pendant toute la période des années 1930 , l'essentiel des produits du palmier vient des palmeraies indigènes qui intéressent presque tous les actifs agricoles de la région élæicole. En 1935, sur les 6848 t d'huile de palme recensées, 4477 t viennent des palmeraies indigènes. Pour ce qui est

9 Free On Board 


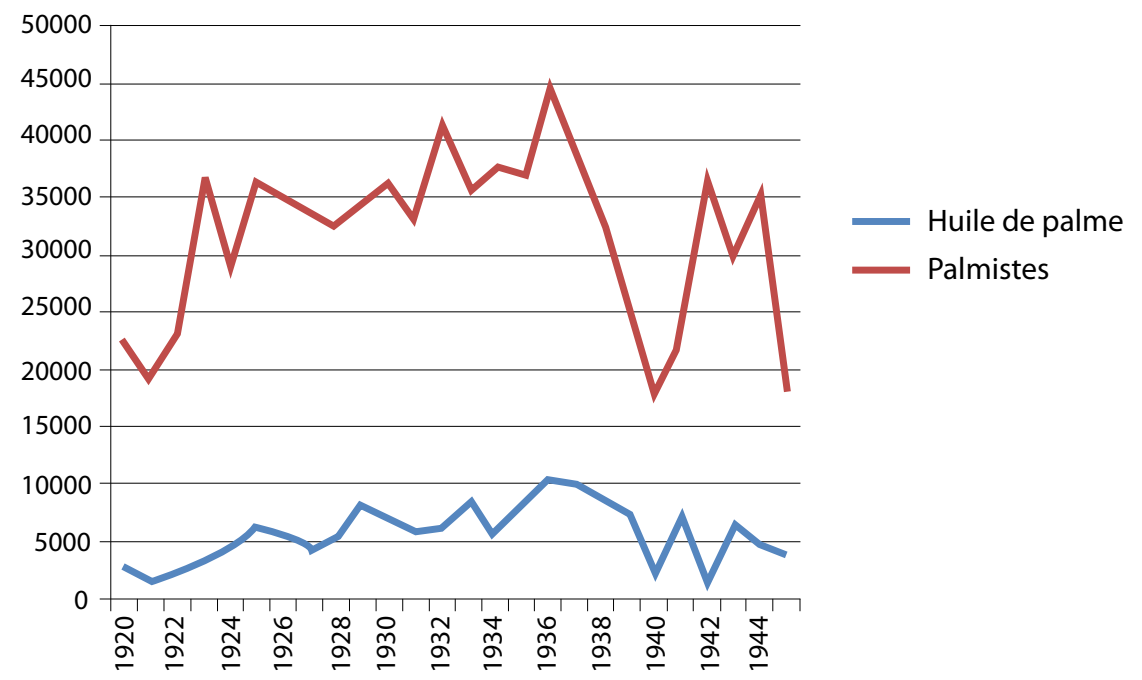

Figure 8 : Évolution des exportations des produits du palmier au Cameroun entre 1920 et 1944 Source : Etoga, 1971

des palmistes, sur $42485 \mathrm{t}$ produites, $37149 \mathrm{t}$ sont issues des contributions indigènes (Etoga, 1971, Bidjeck, 2002) (fig. 9).

L'analyse qui précède montre que, dans le Cameroun sous mandat français, co-existent deux principaux systèmes d'exploitation du palmier à huile :

- la cueillette des régimes de la palmeraie dite « naturelle » par les indigènes qui extraient traditionnellement l'huile par foulage au pied, en consomment une partie, vendent l'autre aux Européens pour l'exportation, ainsi que tous les palmistes dont ils n'ont pas besoin pour la pharmacopée traditionnelle ;

- la culture du palmier à huile dans les plantations coloniales et l'extraction dans des huileries fonctionnant avec des presses hydrauliques.

Les Européens ont également mis en place un système d'exploitation du palmier à huile intermédiaire entre ces deux cas principaux. Il consiste à louer des palmeraies naturelles aux indigènes pour y cueillir les régimes produits, puis à extraire l'huile dans les huileries. Ce système intermédiaire nécessite une proximité suffisante entre la palmeraie naturelle louée et l'huilerie.

Les palmeraies coloniales sont d'anciennes plantations allemandes pour l'essentiel acquises dans les années 1920 et appartenant maintenant aux colons français. Leur production est

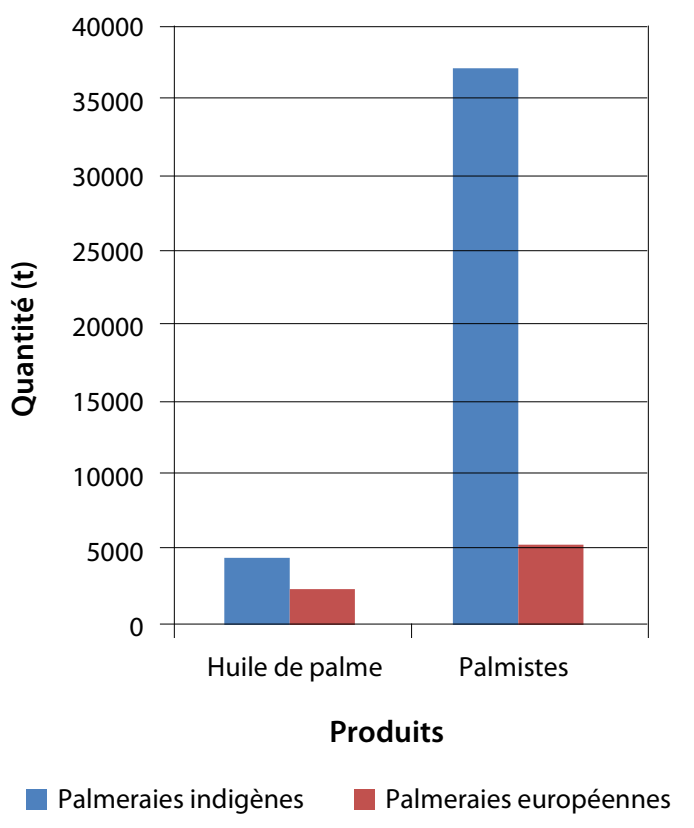

Figure 9: Exportations d'huile de palme et de palmiste au Cameroun en 1935

Source : Etoga, 1971

exclusivement destinée à l'exportation. Cette vocation justifie la localisation de la plupart d'entre elles le long de la voie ferrée, voire à proximité directe des gares ferroviaires qui doivent faciliter l'évacuation de l'huile de palme et des palmistes vers la côte avant d'être exportées vers la métropole. On distingue trois types d'exploitants des palmeraies coloniales. Il y a d'abord les démobilisés, c'est-à-dire les militaires retournés à 
la vie civile et ne souhaitant pas rentrer en France. Certains d'entre eux reprennent d'anciennes palmeraies et huileries allemandes tandis que d'autres préfèrent créer les leurs. En dehors des démobilisés, il y a aussi des commerçants qui possèdent des usines dans la région. Enfin, certaines plantations appartiennent à des sociétés industrielles. C'est le cas la Société Nationale du Cameroun (SNC), dont le siège est alors à Paris et qui gère certaines palmeraies dans la subdivision d'Eséka. Cette société contrôle également un comptoir commercial à Douala, une tonnellerie et des scieries à Manoka (Tjééga, 1974).

Les plantations coloniales changent maintes fois de propriétaire pour des raisons à la fois économiques et historiques. La plantation de Cougoulat illustre bien cette tendance (Tjééga, 1974) : cette plantation appartient d'abord à un planteur allemand du nom de Lassen. Par l'ordonnance du 07 janvier 1924, la plantation de Lassen devient une propriété de l'État. Au mois de novembre de la même année, elle est accordée à la Société Nationale du Cameroun (SNC) alors dirigée par Monsieur Callais. Elle est cédée définitivement à la société le 05 avril 1930 par l'arrêté $N^{\circ} 151$ avant d'être achetée le 31 juillet 1947 par Monsieur Cougoulat.

Sur le plan socioéconomique, les premières palmeraies coloniales se distinguent des palmeraies indigènes par leur vocation exclusivement commerciale, par l'utilisation d'une main d'œuvre exclusivement salariée, et par un accès à la terre par le droit positif afin de disposer d'un usage privé reconnu par l'État colonial. Sur le plan agro-technique, les différences fondamentales concernent le passage à la "plantation " monospécifique qui induit un dispositif de plantation régulier des palmiers, l'entretien des ronds au sol et des couronnes pour faciliter la récolte. Cependant, ces différences agro-techniques ne sont pas systématiques puisque certains colons se contentaient d'exploiter les palmiers ayant poussé spontanément comme le rapporte Franqueville en 1952 : "Comme l'autochtone, le planteur européen s'est longtemps contenté d'exploiter le palmier au moindre coût sans chercher à créer de grandes plantations de rapport."

Sur le plan spatial, les plantations coloniales couvrent deux principales régions, à savoir le Moungo, notamment Dibombari et la Sanaga-
Maritime scindée en trois subdivisions, à savoir la subdivision d'Edéa, la subdivision d'Eséka et la subdivision Babimbi. Dans la subdivision Babimbi qui couvre les arrondissements actuels de Ngambè, de Massok-Song-Lulu et de Ndom, on ne note aucune plantation européenne certainement à cause du relief accidenté et de l'enclavement. Curieusement, malgré ces " handicaps ", c'est à Ndogmakumag dans cette même subdivision que la SAFA installe le siège de sa filière bois (Yomok, 2000). Compte tenu des potentialités élæicoles optimales, de la proximité avec le port de Douala et de la desserte du chemin de fer, c'est finalement dans le Moungo et dans les subdivisions d'Edéa et d'Eséka qu'on retrouve la majorité des plantations coloniales.

Au lendemain de la Deuxième Guerre mondiale, les régions d'Edéa et du Moungo possèdent de vastes plantations coloniales au sein de véritables agro-industries. Dans ces deux régions, la Société des Plantations Réunies de l'Ouest Africain (SPROA), dont le siège est à Gagnoa en Côted'Ivoire, a installé deux plantations et une huilerie à Edéa. L'une des plantations est la Société des Palmeraies de la Ferme Suisse (SPFS) située sur la route Edéa-Kribi. Créée en 1907 et productive à partir de 1910, sa superficie est de 1230 ha en 1952, pour une production moyenne annuelle de $4500 \mathrm{t}$ de régimes, traités au départ dans la propre huilerie de la SPFS, puis à l'usine d'Edéa, approvisionnant ainsi cette huilerie à hauteur de $31 \%$ du tonnage traité au début des années 1950 (Franqueville, 1952). L'autre plantation de la SPROA couvre une superficie de 1200 ha et se trouve à Kompina, dans l'arrondissement de Dibombari sur la route Douala-Nkongsamba. Comme celle de la SPFS, elle est créée pendant la période allemande et n'atteint plus à cette date des rendements élevés ( $4 \mathrm{t} / \mathrm{ha}$ de régimes). Sa production est également expédiée à l'usine d'Edéa et se situe autour de $5000 \mathrm{t}$ de régimes par an au début des années 1950. Les principales plantations du Moungo et de la subdivision d'Edéa sont récapitulées dans le tableau 1 .

Parmi ces plantations, quelques-unes sont agroindustrielles et se concentrent dans la subdivision d'Edéa (Cf. tableau 2).

À cette époque, ces firmes capitalistes aux superficies atteignant jusqu'à 6500 ha sont localisées dans la subdivision d'Edéa, tandis 
Tableau 1 : Les principales plantations coloniales au Cameroun en 1952

\begin{tabular}{llccc}
\hline Plantation & Lieu & Date de création & Superficie (ha) & Production de régimes (t) \\
\hline Kompina & Dibombari & & 1200 & 5000 \\
\hline Ferme suisse & Edéa & 1907 & 1230 & 4500 \\
\hline Edéa & Edéa & 1952 & 489 & 2200 \\
\hline Song Ndong & Edéa & 1952 & 489 & \\
\hline Nkapa & Dibombari & 1952 & 489 & 1100 \\
\hline SMP & Dibombari & 1952 & 4446 & 1250 \\
\hline Bomono et Souza & Dibombari & - & 268 & \\
\hline Guyot & Moungo & - & 108 & 275 \\
\hline IRHO & Dibamba & 1948 & & \\
\hline DEBRABAND & Edéa & & & \\
\hline
\end{tabular}

Source : Franqueville, 1952.

Tableau 2 : Les agro-industries de la subdivision d'Edéa en 1952

\begin{tabular}{lcl}
\hline Sociétés & Superficies $($ ha) & Lieu \\
\hline SAFA & 6500 & Dizanguè \\
\hline SPRAO & 1500 & Edéa \\
\hline DEBRABAND & 275 & Edéa \\
\hline
\end{tabular}

Source : Franqueville, 1952 que la subdivision d'Eséka se caractérise par des entreprises managériales appartenant presqu'exclusivement à des particuliers (Tableau 3). On y distingue deux types de palmeraies européennes en fonction des systèmes de culture mis en place : des palmeraies monospécifiques (Tableau 3) ou plurispécifiques (Tableau 4).

Tableau 3 : Palmeraies européennes monospécifiques de la subdivision d'Eséka dans les années 1950

\begin{tabular}{llcc}
\hline Exploitant & Lieu & Superficies (ha) & Année de création \\
\hline Gramagnac & Ngongoum & 10 & 1937 \\
\hline Mlle David & Ikoadjom & 180 & 1928 \\
\hline Cougoulat & Eséka & 500 & 1924 \\
\hline Fraustey & Minka & 76 & 1928 \\
\hline Ramel & Sodibanga & 200 & - \\
\hline Schüller & Sodibanga & 09 & 1936 \\
\hline \multirow{3}{*}{ Homérides } & Bitchoka & 200 & 1928 \\
\cline { 2 - 4 } & Badjob & 200 & 1928 \\
\cline { 2 - 4 } & llanga & 15 & 1936 \\
\cline { 2 - 4 } & Messondo & 49 & 1936 \\
\cline { 2 - 4 } & Eséka & 32 & 1471 \\
\hline Total & & & 1928 \\
\hline
\end{tabular}


Tableau 4 : Plantations coloniales plurispécifiques d'Eséka dans les années 1950

\begin{tabular}{lllll}
\hline Exploitant & Lieu & Culture & Surfaces (ha) & Année de création \\
\hline Buffet & Ndjock & Palmier, cacao, kolatiers & 375 & - \\
\hline Cluzel & Song Teng & Palmier, café, cacao & 220 & 1929 \\
\hline Homérides & Bitchoka & Palmier/cacao & 420 & 1936 \\
\hline CONCOCAK & Bitend & Palmier/cacao & 200 & 1933 \\
\hline \multicolumn{2}{l}{ Total des superficies } & & $\mathbf{1 2 1 5}$ & \\
\hline
\end{tabular}

Source : ANY, 1950 ; Tjééga, 1974 ; Bidjeck, 2007

Les palmeraies d'Eséka datent pour la majorité des années 1920. Il s'agit le plus souvent d'anciennes plantations monospécifiques allemandes dont l'exploitation est marquée par l'emploi d'une importante main d'œuvre salariée pour la récolte, l'élagage, le nettoyage, la sélection et l'alignement des jeunes palmiers.

À côté des palmeraies monospécifiques, il existe aussi de nombreuses plantations associant le palmier à huile à d'autres cultures (Tableau 4).

À partir de 1949/1950, certaines plantations coloniales sont abandonnées au profit d'autres activités plus rentables notamment de l'exploitation forestière. Il en est ainsi des plantations de Buffet (375 ha) et Fraustey abandonnées par leurs propriétaires au profit de la coupe du bois de chauffe qui alimente les locomotives. Les faibles rendements par hectare de ces palmeraies plantées avec du matériel végétal local peu productif et les chutes très importantes des cours dans les 10 années qui précèdent, sont certainement à l'origine de ces décisions d'abandon.

Les abandons ont dû se généraliser dans la subdivision d'Eséka, car en 1970, les plantations européennes monospécifiques de palmiers qui occupaient 1471 ha dans les années 1950, ne couvrent plus qu'une superficie totale de 502,6 ha, soit 3,05\% seulement des concessions rurales attribuées dans la subdivision. À la même époque, les plantations plurispécifiques couvraient une superficie de 16429 ha, représentant 61,5\% des superficies de concessions rurales accordées. On note une forte implication des missions chrétiennes qui entretiennent plus de 1079 ha (Tjééga, 1974).

Au demeurant, les plantations européennes monospécifiques ont constitué un premier pas vers l'intensification de l'exploitation du palmier à huile au Cameroun par rapport à la cueillette des palmeraies dites "naturelles ». Cependant, à cause des rendements limités, de la fluctuation des cours, des mauvaises conditions de travail des ouvriers (Yomok, 2000) et surtout de la concurrence avec l'exploitation forestière (Tjééga, 1974), la plupart de ces plantations coloniales font faillite, ce qui n'est pas le cas des plantations plurispécifiques.

À côté des plantations européennes coloniales, l'administrateur colonial encourage la création des plantations indigènes qui répondent d'abord à un souci d'amélioration de la commercialisation et de la qualité de l'huile de palme (Etoga, 1971), ce qui nécessite de traiter les régimes rapidement. Pour intéresser les indigènes à la culture du palmier à huile et les encourager à améliorer la qualité de leurs produits, principalement l'huile de palme, les autorités coloniales introduisent l'individualisation de l'exploitation des palmeraies naturelles.

Tout indigène exploitant individuellement une palmeraie naturelle peut désormais en devenir le propriétaire exclusif et se voir attribuer un titre foncier sur ladite parcelle (Etoga, 1971; Kouo, 1979).

Au-delà des objectifs sociaux et économiques, il faut aussi voir dans cette initiative des autorités coloniales qui ont pour mission d'approvisionner la métropole en huile, la volonté de construire des modèles de réussite paysanne dans la région afin que le développement élæicole par les " indigènes " diffuse en tache d'huile. L'incitation et l'encouragement des populations à l'exploitation de cette culture peuvent également être interprétés comme une stratégie de l'administration coloniale pour accroître les recettes fiscales en permettant aux populations d'être solvables pour le paiement des impôts. Il est également question pour le colonisateur de monétariser la production agricole. Du côté des acteurs indigènes, au-delà de la volonté 
de s'approvisionner en produits de première nécessité qui constitue la motivation majeure à la création des palmeraies, les revenus issus de la culture du palmier permettent le paiement des impôts. Les produits du palmier à huile sont à l'époque la principale sinon la seule source de revenus des paysans de la Sanaga-Maritime.

Les plantations indigènes sont ainsi créées par les populations autochtones et notamment les élites ${ }^{10}$ qui s'efforcent de reproduire le modèle colonial. La main d'œuvre est limitée à la famille, aux amis et aux groupes d'entraide sans recours au salariat. Les surfaces plantées sont généralement réduites. Cependant, certains indigènes réussissent à mettre sur pied des plantations de plus de 5 ha. Dans la seule région de Makak, cinq exploitants de plus de 5 ha de palmeraies sont recensés au début des années 1950. Sur les cinq, trois possèdent des presses artisanales (ANY, 1952).

Concernant la première transformation, c'est-àdire l'extraction d'huile, rappelons que jusqu'en 1963, la principale huilerie existante au Cameroun oriental est celle d'Edéa installée en 1953. Elle est gérée par la SPROA. Sa capacité de traitement est supérieure à $30000 \mathrm{t}$ de régimes par an. En 1965, la quantité traitée n'était plus que de $15000 \mathrm{t}$ dont 10000 provenaient des différents secteurs industriels et villageois du périmètre d'Edéa et 5000 de celui de Dibombari. La production se situait autour de $1500 \mathrm{t}$ d'huile de palme et $1100 \mathrm{t}$ de palmistes. Ajoutons la petite huilerie de Kahn, dans l'arrondissement de Ngambé. Sa capacité est évaluée à $1000 \mathrm{t}$ de régimes par an dans les années 1950. Malheureusement, elle ne fonctionne que sporadiquement.

La fin de la Deuxième Guerre mondiale voit également le développement des industries de seconde transformation dans le pays. Elles consistent à élaborer divers produits à partir de l'huile de palme brute et de l'huile de palmistes à l'aide de procédés industriels ou artisanaux. Dans la circonscription d'Eséka qui couvre l'actuel département du Nyong-et-Kéllé, on signale

10 Il convient de noter qu'à cette période l'appartenance à l'élite était subordonnée à la réunion des critères suivants : la possession d'une éducation occidentale et une certaine aisance financière (revenus d'au moins 250 livres). Tardis C. (1956) étudiant les élites de Poro Novo retient le degré de scolarisation tandis que Busia (Op. cit.) propose la population lettrée occupant au moins $10 \%$ de la population totale. l'installation de la société PROPALM à Eséka entre 1946 et 1947 . Cette société produit du savon, jusque-là produit artisanalement et d'utilisation limitée à la population locale.

Cependant la plus importante unité de transformation des produits du palmier dans le Cameroun sous tutelle française reste la savonnerie LA SANAGA DE GALOLMARINES soutenue par des planteurs européens dont M. Coujoulat. Cette savonnerie doit sa dénomination au nom du département de la Sanaga-Maritime dont faisait partie la subdivision d'Eséka en 1947. Cette entreprise emploie 50 salariés au début des années 1950 (Copo-Rey et al., 1968). En dehors du savon de ménage, la SANAGA fabrique aussi des détergents et du savon de toilette. En 1947, sa production est évaluée à $270 \mathrm{t}$ de savon. Cette production atteint 1350 t en 1950 avant de chuter à $30 \mathrm{t}$ seulement au moment de la fermeture de l'entreprise en 1954 principalement à cause de la concurrence de l'exploitation forestière qui a absorbé une partie de sa main d'œuvre limitant ainsi la capacité de production de la savonnerie.

Signalons également la contribution de l'antenne de l'IRHO d'Otélé qui produit également du savon (Mveng, 1963). Par ailleurs, une élite en la personne du Chef Eone Oscar de Matomb s'illustre dans les années 1940 avec la création d'une savonnerie artisanale d'une capacité de 12 t de savon par an (A.N.Y., 1950). Dans la subdivision Babimbi, on note également l'existence de la savonnerie de SAVIDES dans la même période et qui produit plus de $120 \mathrm{t}$ de savon par an (Yomok, 2000).

En 1956, la Compagnie Commerciale Chypriote (CCCh) aujourd'hui connue sous le nom de Complexe Chimique Camerounais (CCC) est créée à Douala. Elle exploite une usine de trituration de palmistes et une savonnerie emploie 196 ouvriers, produit 1178 t d'huile de palmistes, $3500 \mathrm{t}$ de savons et $3100 \mathrm{t}$ de tourteaux de palmistes en 1962 (BEI, 1964).

\subsection{Une production assurée essentiellement par les palmeraies « naturelles »}

En définitive, dans la première moitié du $\mathrm{XX}^{\mathrm{e}}$ siècle, l'histoire est très mouvementée, 


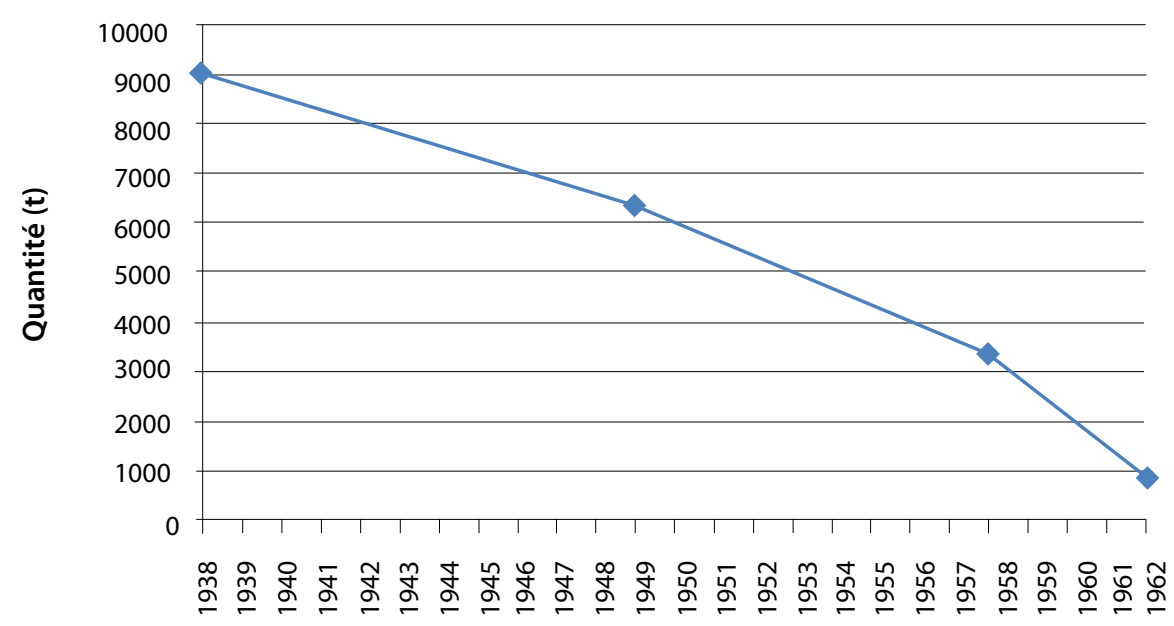

Années

Figure 10 : Évolution des exportations d'huile de palme au Cameroun (1938-1962) Source : BEl, 1964.

marquée successivement par la guerre de 19141918, la crise économique de 1929 et la guerre de 1939-1945, induisant des fluctuations importantes dans les cours mondiaux de l'huile de palme et des palmistes (figure 8), des confiscations de plantations qui n'ont pas encouragé les investissements dans ce secteur.

En effet, à partir des années 1930, les exportations des produits du palmier connaissent une forte régression. Celles de l'huile de palme s'effondrent d'année en année (fig. 10). Les exportations d'amandes de palmistes diminuent également mais dans une moindre proportion passant de $50000 \mathrm{t}$ en 1932 à $36000 \mathrm{t}$ en 1949 et $13000 \mathrm{t}$ en 1958 (BEI, 1964). Cette situation est imputable à la concurrence de la cacaoculture qui détourne une partie des exploitants du palmier à huile et les activités forestières qui détournent la main d'oeuvre. Elle s'explique également par l'utilisation des méthodes et des techniques de cultures extensives car jusqu'au milieu des années 1970, l'huile de palme exportée par le Cameroun provient essentiellement des peuplements naturels surtout dans le Cameroun oriental où l'extraction de l'huile est dominée par des procédés artisanaux traditionnels. Le matériel végétal utilisé jusque-là ne permettait pas d'obtenir des rendements élevés ; l'utilisation des intrants était très limitée, et la majorité des plantations créées pendant la période allemande était très vieille.
Même si l'exploitation en cueillette des palmeraies dites naturelles par les indigènes domine toujours en assurant plus de $90 \%$ de la production d'huile de palme au Cameroun sous administration française, de nouveaux systèmes de culture ont été mis en place par les " colons » allemands puis par les " colons " anglais ou français selon les zones : la culture du palmier à huile en plantations monospécifiques et plurispécifiques. Parallèlement des huileries avec presses hydrauliques plus performantes sont implantées permettant la naissance des premières agro-industries et l'amélioration de la qualité de l'huile, tandis une filière de seconde transformation de l'huile de palme se développait. Au cours de la première moitié du $\mathrm{XX}^{\mathrm{e}}$ siècle, la filière palmier à huile se caractérise donc par la juxtaposition d'une part, de plantations coloniales agro-industrielles et managériales dont la production monte très doucement en puissance, et d'autre part, de l'exploitation traditionnelle de la palmeraie dite " naturelle " par les indigènes qui assurent encore la grande majorité de la production du Cameroun. Dans les régions élæicoles du Cameroun, les " colons » dépendent des " indigènes " pour approvisionner en huile la métropole, tandis que les indigènes accèdent à la monétarisation et par là même aux biens de consommation apportés par les " colons", en vendant les produits issus de leur exploitation. Les plantations managériales sont celles qui ont le moins bien résisté aux fluctuations des cours mondiaux consécutives aux évènements historiques de la première moitié du $\mathrm{XX}^{\mathrm{e}}$ siècle. 


\section{La modernisation du secteur élæicole camerounais}

\subsection{Une forte implication de l'État dans la modernisation de l'élæiculture}

La modernisation de la filière palmier à huile commence au début de la Deuxième Guerre mondiale et s'accélère après les indépendances. Elle débute avec la production de matériel végétal sélectionné de type tenera nettement plus performant que la matériel local de type dura, et se poursuit avec la création des palmeraies et des usines industrielles autour desquelles se développent des plantations villageoises selon le modèle des " plans palmier " mis en place dans plusieurs pays africains, c'est-à-dire l'implantation de palmeraies villageoises sur les propres terres de l'agriculteur bénéficiaire du crédit de plantation. Cette dynamique est soutenue par une forte volonté politique de l'État à travers la mise en œuvre de véritables plans de développement dès les indépendances au début des années 1960 et la création des institutions de crédit rural, ainsi que d'agro-industries publiques, sociétés de développement en charge de l'appui technique et du recouvrement des crédits de plantation.

\subsection{La création des centres de recherche spécialisés}

Dans le Cameroun francophone, la recherche sur le palmier à huile commence avec la création de l'IRHO ${ }^{11}$ en 1939. L'Institut a entre autres missions la modernisation de la culture du palmier à huile et l'installation d'huileries afin d'accroître la production d'huile de palme au Cameroun (Mveng, 1985). Dans cette perspective, une station expérimentale est installée dans l'arrondissement de la Dibamba. Ce centre expérimental commence à fonctionner en 1947. Ses missions englobent l'amélioration génétique du palmier à huile et des pratiques culturales en palmeraie afin de

11 La création de l'IRHO répond au plan de développement de la production des matières grasses de 1945 . développer la production, le renouvellement du verger et de vulgariser les innovations techniques produites. Les premiers plants de la station de la Dibamba sont produits en 1950 (Botele Ndiomo, 1973). Sur financement du Fonds International pour le Développement Economique et Social des Territoires d'Outre-Mer (FIDES), l'IRHO construit deux huileries modernes, l'une à Dibombari d'une capacité de 2000 t de régimes / an et l'autre à Edéa d'une capacité de $1000 \mathrm{t}$ de régimes/an. En plus, l'institut procède à la création des plantations autour de Dschang, Bafia, Kribi et Edéa. D’autres huileries sont créées à Otélé, Pongo et Nkankanzok (Mveng, 1985). Pour tester les premiers plants produits par le centre de la Dibamba et afin d'impliquer les populations dans la culture du palmier à huile sélectionné, les autorités coloniales mettent sur pied le programme de plantations familiales de palmiers sélectionnés.

Dans le Cameroun anglophone, la CDC crée également un centre de recherche à Ekona en 1953 produisant entre autres matériels végétaux des plants de palmier à huile sélectionnés (Esua F., 2001). Les plants de ce centre sont essentiellement utilisés dans les plantations de l'entreprise. En 1971, le département de recherche de la PAMOL situé à Ekondo Titi, le Lobe Research Department, commence la production commerciale de semences améliorées.

\subsection{Le programme de plantations familiales de palmiers sélectionnés}

Ce programme est mis en place en 1952 dans la région de Dibombari sur un échantillon de 850 palmeraies villageoises. Dans la SanagaMaritime, des essais analogues sont faits à Mom, Messondo et Digombi (Champaud, 1973). C'est un projet pilote de vulgarisation des plants sélectionnés de palmier à huile (Elong, 2008) mis en ouvre par les services d'agriculture en collaboration avec l'IRHO. Â travers ce 


\section{Encadré 2 : Le Secteur de Modernisation}

Les secteurs de modernisation sont des établissements publics autonomes, administrés par un Conseil d'Administration de 25 à 46 membres. Les secteurs constituent des organismes de vulgarisation agricole. Ils doivent promouvoir par l'exemple et la vulgarisation, le développement et la production agricole. Le financement des secteurs est assuré principalement par le FIDES et le PAC jusqu'en 1960, puis est progressivement pris en charge par le budget national. Il en résulte un amenuisement des ressources qui tend à limiter les moyens d'action de ces organismes, d'autant qu'il n'est pas possible d'étendre leur activité à la commercialisation des produits. Chaque secteur se subdivise en sous-secteurs à raison d'un au moins par département, et dont l'administration est confiée à un Comité de Direction. Les sous-secteurs se subdivisent eux-mêmes en postes agricoles qui constituent l'unité d'encadrement de base des producteurs, chaque poste regroupant 7 à 12000 habitants. Les secteurs de modernisation couvraient 5 régions :

1. Le SEM-Centre, dont la direction est à Yaoundé, oriente son action principalement en faveur du cacao, mais aussi en faveur du café.

2. Le SEM-Maritime, dont la direction est à Douala oriente son action à la fois sur le cacao, le café, le palmier à huile, le coprah et les cultures vivrières.

3. Le SEMCA ou Secteur des Cultures d'Altitude (Bamoun et Bamiléké), dont la direction est primitivement fixée à Dschang, et doit être transférée à Foumban lors des troubles en pays Bamiléké, a pour but essentiel d'étudier les conditions de mise en valeur des périmètres ruraux. Les activités de ce secteur sont ainsi concentrées sur le pays Bamoun et portent sur l'amélioration du caféier arabica en altitude et le développement de la culture du caféier robusta dans les zones plus basses.

4. Le SEM-Est, dont la direction est à Bertoua, s'oriente vers la mise en valeur des terres nouvelles. II se consacre au développement de la culture du cacao, du café et de l'arachide, ainsi qu'à la formation d'animateurs ruraux.

5. Le SEM-Nord, dont la direction est à Garoua, est lui-même subdivisé non seulement en sous-secteurs géographiques, mais aussi en sous-secteurs spécialisés (riziculture, hydraulique rurale, chasse).

Source : BEI, 1964

projet, l'administration entend contribuer à l'augmentation des exportations d'huile de palme en introduisant dans le bassin élæicole camerounais un matériel végétal performant tout en rajeunissant le verger. Parallèlement, des actions de développement cherchent à intéresser les paysans à la culture du palmier à huile afin d'augmenter la production nationale, de développer les zones rurales et d'approvisionner les huileries en régimes. La seule condition exigée pour participer à ce programme d'appui à l'élæiculture est de posséder un terrain dont la propriété foncière est reconnue par la communauté villageoise. Les travaux préalables (défrichement, piquetage, trouaison), le transport et la mise en terre des plants sont pris en charge par le Secteur de Modernisation du Palmier (SMP), Cf. encadré 2.

Les palmeraies créées dans le cadre de ce programme sont de véritables plantations « clés en main » : elles sont entretenues pendant la période juvénile par le SMP, puis remises aux propriétaires de la terre dès la production des premiers régimes et sans contrepartie financière (Elong, 2008). Dans la Dibombari par exemple, ce programme permet de créer plus de 846 ha de plantations de palmiers sélectionnés entre 1952 et $1958^{12}$. En 1959, 327 ha supplémentaires sont plantés. Finalement, entre 1952 et 1962, 1191 ha de palmeraies sont créées dans ce seul arrondissement dans la même localité (Elong, 2008). Cependant, dès la campagne 1959-1960, le SMP abandonne les palmeraies villageoises " clés en main " et propose aux paysans d'acheter des plants sélectionnés. Il est clair que cette nouvelle stratégie demande une plus grande participation des paysans qui doivent désormais non seulement acheter les plants, mais aussi supporter le coût de la mise en place et de l'entretien important des plantations pendant la phase juvénile.

12 Botele Ndjiomo ( 1973) en a recensé 850. 
Petit à petit, l'engouement de départ manifesté par les paysans se transforme en déception au point que bon nombre d'entre eux abandonnent le projet. Finalement, ni l'exploitation traditionnelle, ni les plantations coloniales ou les récentes plantations villageoises de palmiers sélectionnés ne parviennent à satisfaire la demande interne croissante en huile.

\subsection{Les grandes actions de développement élæicole du Cameroun indépendant}

La création de plantations industrielles et villageoises ainsi que d'huileries industrielles de grandes capacités $(20 \mathrm{t} / \mathrm{h})$ se fait dans le cadre de la mise en œuvre des plans de développement surtout dans le Cameroun oriental dominé jusque-là par les palmeraies naturelles.

\subsubsection{Le plan quinquennal de développement du Cameroun sous tutelle anglaise}

Au début des années 1950, les autorités anglaises se rendent compte que l'administration nigériane se soucie peu du développement du territoire camerounais. Elles mettent sur pied entre 1955 et 1960, un plan quinquennal de développement du Cameroun sous tutelle anglaise. Ce programme se fonde principalement sur les investissements de la CDC (Esua, 2001). Grâce à ce programme, les productions d'huile de palme et de palmistes connaissent une légère amélioration dans le Cameroun sous tutelle anglaise. En 1959, les surfaces élæicoles de la CDC atteignent 7200 ha contre 6462 ha en 1951 (Esua, 2001). Les exportations d'huile de palme et de palmistes correspondent respectivement à $8257 \mathrm{t}$ et $5590 \mathrm{t}$ en 1960, soit une augmentation respective de $28 \%$ et $13 \%$ par rapport aux performances de 1955 . Dans tout le Cameroun occidental, les exportations d'huile de palme sont estimées à plus de $9600 \mathrm{t}$ en 1961 contre 5500 t en 1956. En 1961, la part du Cameroun sous tutelle anglaise dans les exportations des produits du palmier est de 56,4\% des échanges du Cameroun fédéral (BEI, 1964). Mais globalement, tous ces programmes initiés avant les indépendances tant dans le Cameroun sous tutelle française que dans le Cameroun sous tutelle anglaise n'empêchent pas la baisse de la production d'huile de palme et de palmistes. Au début des années 1960, la culture et l'exploitation du palmier entrent en stagnation. La part des produits du palmier dans les exportations du Cameroun fédéral baisse considérablement. Entre 1955 et 1958, les produits du palmier ne représentaient que $10 \%$ des exportations du pays et en 1962 , ce taux chute à $2 \%$ seulement (BEI, 1964). L'une des causes de cette débâcle est la baisse des prix des produits du palmier qui ne fournit pas des revenus élevés aux populations. À cela s'ajoute le vieillissement du verger et la concurrence du cacao qui fait l'objet d'un grand programme gouvernemental de développement sans oublier l'exploitation forestière qui est jugée plus rémunératrice de la journée de travail par les ouvriers agricoles. Il faut donc penser un projet plus vaste, plus ambitieux et plus structurant pouvant avoir d'importants effets d'entraînement. C'est ainsi que dans le cadre de la mise en ouvre du troisième plan quinquennal de développement déroulé de l'exercice 1970/1971 à celui de 1975/1976, le gouvernement met sur pied le premier grand plan de développement du palmier à huile au Cameroun.

\subsubsection{Le plan palmier du Cameroun}

Le plan palmier est le plus important programme de développement du palmier à huile mis en œuvre au Cameroun après les indépendances. Le plan palmier poursuit principalement deux objectifs, à savoir satisfaire les besoins nationaux en matières grasses puis exporter les excédents pour générer des devises et promouvoir le développement local, régional et national.

L'un des principaux objectifs du plan palmier est d'abord de combler le déficit interne en huile. En effet, l'huile de palme constitue la source idéale de lipides pour une grande partie de la population. Son prix bas lui permet d'être à la portée du Camerounais moyen. Cependant, les besoins nationaux en matières grasses ne cessent de croître (fig. 11).

Au cours de la période allant de 1920 à 1980, les besoins annuels en huile de palme augmentent régulièrement d'en moyenne $11 \%$ par décennie. Parallèlement, Tjééga (1974) évalue l'augmentation de la consommation d'huile de palme à environ $3 \%$ par an. Cette augmentation 


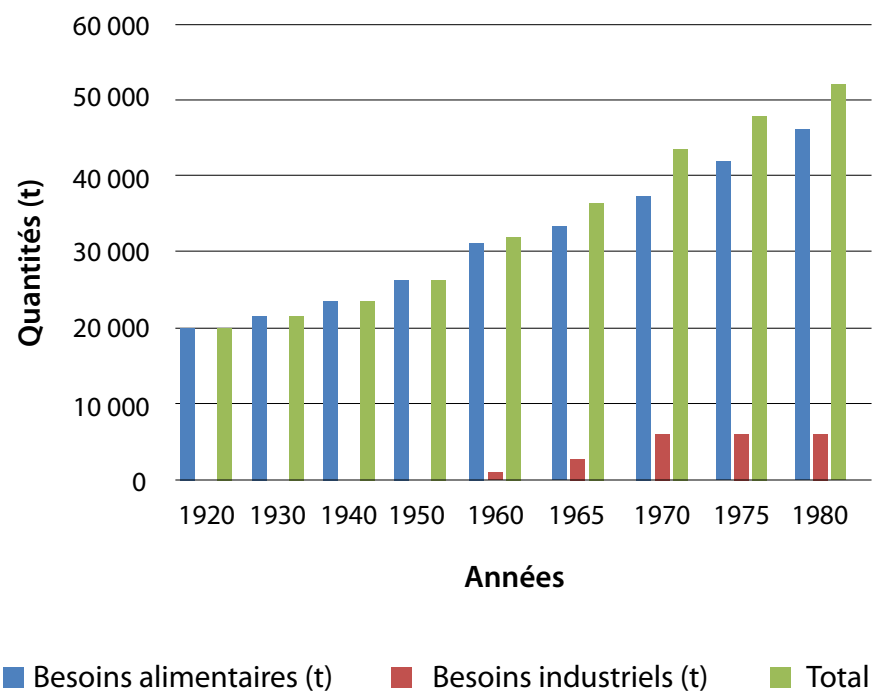

Figure 11 : La demande intérieure en huile de palme au Cameroun (1920-1980) Source :Tjééga, 1974

est liée à l'accroissement de la population et à la croissance de la demande industrielle, principalement par le Complexe Chimique Camerounais (CCC) qui transforme aussi bien l'huile de palme que les palmistes.

Outre le souci de satisfaire le marché intérieur en huile, les autorités camerounaises entendent également faire du plan palmier un projet de développement local et régional dans l'optique d'atténuer les disparités régionales. Dans les régions de forte tradition élæicole comme la Sanaga-Maritime, le Nyong-et-Kéllé, Dibombari et le Sud-Ouest, il est question d'améliorer les revenus des planteurs et de relever le niveau de vie des populations. Il s'agit également de lutter contre l'exode rural en maintenant sur place une population fortement scolarisée et de plus en plus attirée par la ville où elle rêve de trouver des emplois salariés et rémunérateurs. Le plan palmier se présente ainsi comme une aubaine pour cette population, soit à travers les emplois directs que le plan va créer dans les agro-industries, soit à travers des emplois indirects liés au développement des palmeraies villageoises.

Par ailleurs, le plan palmier est également un important canal de diffusion de diverses innovations agricoles au rang desquelles on trouve le palmier sélectionné, l'utilisation d'engrais et de produits phytosanitaires. C'est dans cette perspective que l'ex-président Ahidjo lors d'un discours prononcé en 1973 déclare : "La CDC est une entreprise agro-industrielle importante. Elle doit servir d'exemple et stimuler le progrès des populations aux alentours de ses plantations... [La population locale] devrait aspirer à créer de petites plantations villageoises satellites autour des plantations, où elle pourrait apprendre et appliquer des méthodes et des techniques agricoles modernes ». Et le décret $n^{\circ} 731597$ portant réorganisation de la CDC, daté de la même année, établit comme l'un des objectifs de la CDC d'assister « les petits planteurs ou des groupes de petits planteurs dans la culture des mêmes produits que ceux cultivés par la CDC dans les zones de collecte autour des huileries qu'elle gère. » (Koenings, 1979). Ces deux extraits donnent déjà une idée de la place centrale que doivent jouer les agro-industries, sociétés publiques de développement dans la mise en place du plan palmier.

Le plan se déroule en deux phases. La première phase du projet va de 1969 à 1976 tandis que la seconde est mise en œuvre entre 1976 et 1981.

\section{Le premier volet du plan palmier}

Le premier volet du plan palmier est la mise en œuvre du projet élæicole du Cameroun oriental qui 
correspond à la première tranche des travaux de la SOCAPALM. Au départ, sa durée d'exécution est fixée à sept ans et demi, c'est-à-dire de 1968/69 à 1975. Lors de l'évaluation du projet en 1972, la durée du projet est prolongée à neuf ans à cause de l'irrégularité des approvisionnements en jeunes plants, des pénuries de main-d'œuvre temporaire et des lourdes pertes infligées par les rongeurs aux jeunes palmiers non protégés qui ont nécessité la replantation de 1200 ha. Finalement, ce premier volet débute donc en 1968/69 et prend fin en 1976/77 au lieu de 1974/1975.

Le principal objectif de ce volet est d'accroître la production des plantations industrielles de palmiers à huile au Cameroun en créant une agroindustrie publique, société de développement maître d'œuvre de programmes de développement de plantations villageoises. À cet objectif, se greffent les objectifs spécifiques suivants :

- la création de 8500 ha de palmeraies industrielles dont 6000 ha à M'Bongo et 2500 ha à Eséka,

- la construction d'une huilerie sur chacune de ces deux plantations,

- le développement des infrastructures routières nécessaires à l'évacuation de l'huile et à la collecte de la production dans les plantations.

C'est dans cette optique que par le décret $\mathrm{N}^{\circ}$ 68/ DF/451 du 23 novembre 1968, la Société des palmeraies de M'Bongo et d'Eséka (SOPAME) est créée pour gérer ces deux plantations ainsi que leurs huileries. C'est une société de développement ${ }^{13}$ placée sous le contrôle de l'autorité publique. En tant que telle, elle exécute

13 Les sociétés de développement sont instituées par la loi $\mathrm{N}^{\circ}$ 68/LF/9 du 11 juin 1968 qui définit également leur cadre d'opérationnalisation. Leur fonctionnement est régi par le décret $\mathrm{N}^{\circ}$ 68/DF/451 du 23 novembre 1968. En tant que Société de développement la SOPAME, devenue SOCAPALM est assujettie aux dispositions ci-après de la loi sur les Sociétés de développement :

- au moins $20 \%$ de son capital social doit être détenu par l'État, des organismes publics et/ou d'autres sociétés de développement ;

- l'autorisation peut être donnée par décret pour l'inscription d'une société de développement dans le Code des investissements du Cameroun ou l'attribution d'un monopole industriel et/ou commercial ;

- la société est assujettie au code de commerce du Cameroun ;

- Le contrôle de l'État s'exerce par la nomination, par le Ministre de l'agriculture, d'un Commissaire de gouvernement pour la société (Banque mondiale, 1977). les plans de développement économique et social dans les sites d'accueil. Dans cette logique, la mise en ouvre du premier plan palmier du Cameroun oriental incombe à la SOPAME (BIRD/ AID, 1974).

Ces objectifs sont conformes à la stratégie de développement du gouvernement exposés dans le troisième plan quinquennal (1971/72-1975/76) qui prévoit la création de deux nouvelles palmeraies à Dibombari et à Edéa grâce au financement du Fonds Européen de Développement (FED). Dans le cadre de ce plan, 6000 ha de palmiers à huile sont plantés à Dibombari, et 500 ha à Edéa. Du fait de cette extension des palmeraies industrielles dans le Moungo, la SOPAME est renommée Société des Palmeraies du Cameroun Oriental (SOPACOR) par décret $\mathrm{N}^{\circ} 72 / \mathrm{DF} / 4$ du 03 janvier 1972. Suite à la réunification des deux Cameroun intervenue le 20 mai 1972, la SOPACOR devient la Société Camerounaise de Palmeraies (SOCAPALM) par décret $\mathrm{N}^{\circ} 73 / 32 \mathrm{du}$ 29 janvier 1973. Cette société a pour mission de créer et d'exploiter les palmeraies, de traiter et de vendre l'huile de palme et les palmistes et d'aider les petits planteurs qui s'intéressent à la culture du palmier à huile lorsqu'ils sont situés dans le bassin d'approvisionnement de ses huileries. La fin juin 1976 marque l'achèvement de la première phase du projet SOCAPALM, plus de 8280 ha de palmeraies industrielles étant plantés. Ils sont complétés au cours de l'exercice 1976/1977 par 380 ha supplémentaires portant le total des surfaces plantées à 8660 ha (Banque mondiale, 1977).

Entre temps, en 1974, la Société Africaine Forestière et Agricole du Cameroun (SAFACAM) qui exploite les plantations d'hévéa dans la zone de Dizangué depuis 1897, décide de diversifier ses activités et convertit certaines de ses surfaces en palmeraies (Ngom, 2013) et en implantant une huilerie d'une capacité de $10 \mathrm{t} / \mathrm{h}$.

Dans le Cameroun sous tutelle anglaise, la mise en ouvre de ce premier volet du plan palmier s'est traduite par le programme de développement régional de la "Cameroons Development Corporation $»^{14}$ (BEI, 1964). En effet, dès 1964, la CDC déroule un projet régional à long terme appelé CAMDEV avec l'aide du Groupe

14 C'est après l'indépendance que Cameroons devient Cameroon. 
de la Banque mondiale et d'autres bailleurs de fonds internationaux. La première phase de ce programme connu sous le nom de CAMDEV 1 démarre en 1964 et s'achève en 1974 grâce principalement aux prêts de la Commonwealth Development Corporation (COMDEV) ${ }^{15} \mathrm{de}$ trois (3) millions de livres et du consortium BIRD-AID-FED (6 milliards de francs CFA). Il vise principalement l'augmentation de la production et de la rentabilité de la CDC et le renforcement de sa structure financière. Ce projet connaît un succès total d'après le rapport d'évaluation commandité par la Banque mondiale en novembre 1965 : "This is a successful project; without it, CAMDEV would in all probability have foundered, whereas with Bank assistance its oil palm and rubber operations have become competitive in the world markets.» Et au rapport d'audit de la CDC Nº 1752, du 12 octobre 1977 d'ajouter : " enormously successful in improving the company's financial structure and operation - a principal objective of the Bank's aid to CAMDEV ». En 1976 à la fin de CAMDEV 1, la CDC enregistre une augmentation nette de ses surfaces de palmeraies de 5800 ha. Sur le plan financier, le plan de financement du projet est couvert à $88 \%$. Le rapport de la Banque mondiale cité ci-dessus indique que la CDC est capable $d$ 'assurer les financements restants sur fonds propres (Banque mondiale, Cameroon Appraisal of a Second CAMDEV Project, 1977).

\section{Le deuxième volet du plan palmier}

C'est le plan palmier proprement dit. Il est réalisé entre 1976 et 1981 et s'inscrit dans le cadre de la mise en ouvre du quatrième plan quinquennal de développement du Cameroun. Ce projet intéresse prioritairement les deux sociétés d'État de l'époque, à savoir la SOCAPALM au Cameroun oriental et la CDC au Cameroun occidental, maîtres d'œuvre du projet. Au Cameroun oriental, ce deuxième volet du plan palmier coïncide avec la mise en œuvre du projet CAMDEV II (19781982) dont les principaux objectifs tournent autour de l'augmentation de la production et de l'efficacité technique et managériale de la CDC, l'introduction d'un programme de plantations

15 Il s'agit d'une banque britannique. Elle fait partie des principaux actionnaires de la Cameroons Development Corporation avant les indépendances au début des années 1960. villageoises et la préparation d'une étude régionale pour le développement futur de la CDC et des localités environnantes. De manière spécifique, il s'agit de créer 1000 ha de palmeraies villageoises et l'extension des plantations industrielles de palmier à huile de 600 ha (Banque mondiale, Appraisal of a Second CAMDEV Project, 1977).

Dans le Cameroun oriental, le deuxième volet du plan palmier constitue le prolongement du projet SOCAPALM. Il poursuit principalement deux objectifs, à savoir :

- la mise en ouvre d'un programme de développement des palmeraies villageoises, parallèlement aux plantations industrielles déjà en exploitation ;

- l'amorce de la première phase d'une nouvelle plantation industrielle de palmiers à huile, dans la Kienké au Sud-Ouest du pays s'étendant à terme sur 12000 ha avec les plantations villageoises environnantes.

De manière spécifique, le projet prévoit entre autres objectifs :

- l'implantation à Kienké de 6000 ha de palmeraies sélectionnées à fort rendement ainsi que leur entretien pendant la période de cinq ans du projet ;

- la mise en place des infrastructures nécessaires à la création d'un complexe industriel, y compris la première phase de construction d'une usine de traitement d'une capacité de $40 \mathrm{t} / \mathrm{h}$;

- la création de 2000 ha de palmeraies villageoises sélectionnées implantées par les agriculteurs sur leurs propres terres ;

- l'extension de la palmeraie industrielle de Mbongo de 1000 ha (Banque mondiale, Rapport d'évaluation du deuxième projet SOCAPALM, 1977).

La mise en ouvre du programme de plantations villageoises dans toute la République Unie du Cameroun est financée par le Fonds National de Développement Rural (FONADER).

\subsection{Le FONADER et la mise en œuvre du programme de développement des plantations villageoises}

Le FONADER est créé le 28 août 1973 par le Décret n ${ }^{\circ} 73 / 496$. C'est une banque de développement rural, essentiellement 
approvisionnée par le Fonds de Stabilisation des Prix ${ }^{16}$ et certaines allocations budgétaires de l'État unitaire. Les principales missions du FONADER sont les suivantes :

- l'administration, le stockage et la distribution des intrants agricoles subventionnés ;

- la promotion et la distribution du crédit agricole ;

- le financement et le suivi de certains projets de développement ;

- le recouvrement indirect des crédits de plantation par l'intermédiaire des sociétés publiques de développement, maîtres d'œuvre du programme de développement de plantations villageoises.

Le FONADER n'a pas de bureaux déconcentrés. Il utilise les services des institutions publiques et des sociétés de développement existantes pour remplir ses missions. Ainsi pour le cacao, il opère par l'intermédiaire de la Société de Développement du Cacao (SODECAO). Pour ce qui est du palmier à huile, le FONADER a recours aux services de la SOCAPALM et de la CDC qui sont des sociétés de développement dotées d'une autonomie financière.

Le FONADER est un véritable organisme de financement du monde rural ayant la charge de distribuer les diverses subventions nationales en direction du monde rural. Le fonds accorde des crédits aux particuliers, aux groupements de pré-coopérateurs comme les Groupements d'Agriculteurs Modernes (GAM) ainsi qu'aux coopératives et aux sociétés de développement. Dans le cadre du programme de plantations villageoises de palmier à huile, les financements du FONADER sont gérées par la SOCAPALM, la CDC et plus tard la PAMOL reprise par l'État du Cameroun en 1980. Ces sociétés de développement identifient les planteurs bénéficiaires du projet dont l'adhésion est soumise à la signature d'un contrat avec l'une des agroindustries ci-dessus mentionnées.

16 Ce fond était alimenté par les excédents des ventes des principaux produits agricoles de base tels que le cacao, le café et le coton.

\subsubsection{Le contrat entre les planteurs et les agro-industries ${ }^{17}$}

Pour être bénéficiaire du projet de développement des plantations villageoises, le paysan est assujetti aux quatre conditions suivantes :

- être de nationalité camerounaise et tirer au moins $75 \%$ de ses revenus de l'agriculture ;

- avoir entre 25 et 40 ans et être physiquement capable d'exercer des travaux dans la plantation. Des personnes plus âgées, mais disposant de jeunes gens à leur disposition peuvent être également retenues ;

- disposer de terres aptes à être mises en valeur dans les conditions suivantes : les plantations doivent être situées dans un rayon de $25 \mathrm{~km}$ de l'huilerie, elles doivent être à une distance n'excédant pas 500 mètres d'une route carrossable ou d'une piste ; le propriétaire doit exercer un droit sur la terre couvrant une période d'au moins 24 ans;

- effectuer l'abattage de la végétation sur la parcelle identifiée pour y implanter une palmeraie villageoise.

Chaque bénéficiaire du projet reçoit une assistance technique et financière pour une superficie de 1 à 2 ha au cours de la première année. Cette superficie peut être élargie d'un hectare par an, jusqu'à un maximum de 5 ha. Dans l'hypothèse de l'utilisation d'une main d'œuvre salariée, la Banque mondiale évalue le coût de création d'un hectare à 82000 francs CFA en 1976 (Banque mondiale, évaluation du deuxième projet SOCAPALM, 1977). Les bénéficiaires du projet contractent un crédit de plantation auprès de l'agro-industrie qui les encadre techniquement à un taux d'intérêt de $9 \%$ par an avec un remboursement différé pendant 6 ans puis qui s'étale d'après un modèle de la septième à la treizième année suivant la plantation. L'agro-industrie qui collecte les régimes une fois la parcelle entrée en production est chargée du recouvrement du crédit de plantation pour le compte du FONADER. Le contrat qui couvre une période de 27 ans à partir de l'année de défrichage, oblige le planteur :

- à vendre ses régimes à l'agro-industrie avec laquelle il est en contrat ;

17 Pour plus de détails, lire le Rapport d'évaluation du deuxième projet SOCAPALM de la Banque mondiale(1977), le rapport d'évaluation du projet CAMDEV II intitulé Appraisal of a Second CAMDEV Project, 1977, Koenings, 1993. 
- à assister à toutes les séances de formation et d'information sur les techniques agricoles;

- suivre scrupuleusement les recommandations de l'agro-industrie sur le suivi et l'entretien de la plantation ;

- à s'acquitter de toutes ses dettes.

Au cas où un planteur ne respecte pas les conditions du contrat, l'agro-industrie, après un certain nombre d'avertissements écrits, transmet le dossier du débiteur défaillant au FONADER qui procède à la saisie de la plantation couverte par le contrat.

Pour sa part, l'agro-industrie s'engage à :

- apporter une assistance technique au champ (effectuer le piquetage de la parcelle, montrer comment planter, semer la plante de couverture, épandre les engrais, récolter les régimes...) et sous forme de formation ;

- fournir les intrants et matériels nécessaires à l'implantation de la parcelle (matériel végétal, grillage de protection contre les rongeurs, semences de légumineuse de couverture) puis sa conduite juvénile (engrais azoté), son entrée en production (perche de récolte) ;

- pendant la période juvénile (maximum 4 ans), le planteur reçoit en espèces un montant compris entre 56900 et 82000 francs CFA, représentant ses frais d'entretien de la parcelle (Koenings, 1993) ;

- une fois les parcelles en production, assurer la collecte des régimes bord champ et entretenir les pistes d'accès aux plantations; le coût de ce service est déduit du prix d'achat des régimes par l'huilerie (5000 francs CFA/t de régimes) ;

- payer mensuellement le paysan pour les régimes livrés ;

- assurer le recouvrement des crédits de plantation pour le FONADER ;

- fournir aux planteurs ayant remboursé la totalité des dettes contractées, un certificat enregistré au Bureau foncier pour les surfaces sous plantation (Banque mondiale, évaluation du deuxième projet SOCAPALM, 1977).

Dans chaque agro-industrie, un département administratif dédié aux plantations villageoises est créé sur recommandation de la Banque mondiale. Notons que le contrat ne prévoit aucune sanction en cas de non-respect de ses obligations par l'agro-industrie.
Le FONADER, avec le programme de plantations villageoises qu'il finance, est considéré comme le moteur du développement rural et élæicole. Dans le Sud-Ouest par exemple, la surface cumulée de plantations villageoises de palmier à huile créées de 1978 à 1987 avec l'aide du FONADER est estimée à 3275 ha dans le bassin d'approvisionnement de la CDC tandis que le nombre de planteurs villageois dépasse 780 (CDC, annual report 1987, cité par Koenings, 1993). Tout porte à croire que ce chiffre peut dépasser 1000 participants au projet si on tient compte des données de 1981, 1982 et 1983 qui ne figurent pas dans le rapport exploité par l'auteur. En 1993, lors de la table ronde de Limbé, on compte plus de 3100 planteurs encadrés par le FONADER et ayant bénéficié du programme de plantations villageoises. Les superficies plantées s'évaluent à plus de 13000 ha (Bakoumé et al., 2002).

Malheureusement, le FONADER, tout comme ses prédécesseurs tombe en faillite en 1990 et est définitivement liquidé en 1991. Plusieurs facteurs expliquent cette banqueroute du fonds.

\subsubsection{La faillite du FONADER}

Les causes de la chute du FONADER sont de deux ordres. Certaines sont internes tandis que d'autres sont externes.

Parmi les facteurs ayant entraîné la chute du FONADER, il y a d'abord son fonctionnement interne. En effet, à cause d'une centralisation excessive, cet organisme éprouve de sérieuses difficultés d'organisation. L'absence de représentations régionales et locales réduit ses capacités d'intervention. Les lourdeurs administratives entraînent des retards dans la distribution des subventions auprès des producteurs villageois.

Initialement prévu pour distribuer le crédit dans le monde rural, le FONADER est devenu plus tard un organisme d'intervention de l'État dans d'autres secteurs économiques parfois non productifs. Le fonds profite finalement plus à d'autres acteurs qu'aux ruraux. Fouda Moulende (2003) parle même de l'exclusion des paysans du programme FONADER et montre que pour le crédit adhérent du FONADER par exemple, seulement $10 \%$ 
de la ligne de crédit (déjà très modeste en ellemême) sont allés aux petits producteurs. Tchatat (1984, cité par Fouda Moulende, 2003) explique cette situation par les nombreux blocages qu'a rencontré le FONADER dans l'accomplissement de ses missions par : "la faible capacité de remboursement des paysans, à la complexité de procédures visant à réduire les impayés, la priorité accordée au crédit social par rapport au crédit à la production, la structuration insuffisante du monde rural, l'inadaptation statutaire, (...) à ses faibles ressources. "

Le programme de plantations villageoises financé par le FONADER est en principe réservé à $90 \%$ aux paysans, c'est-à-dire à ceux qui tirent $75 \%$ de leurs revenus de l'agriculture. De nombreuses élites urbaines et rurales (cadres des agro-industries maittres d'œuvre du projet) ont cependant profité de ce projet pour créer leurs propres palmeraies, privant les ruraux d'un bien qui leur était statutairement destiné. Elong (2003) signale ainsi que dans le Bas-Moungo, les élites possèdent $70 \%$ de la superficie totale des palmeraies villageoises en 1989. Presque toutes les palmeraies des élites urbaines et rurales créées dans les années 1970 et 1980 ont bénéficié des financements du FONADER. Enfin, notons également le manque de transparence dans le suivi du remboursement des crédits de plantation que les paysans ont l'impression de rembourser éternellement. En effet, le modèle de remboursement entre la septième et la treizième année n'a pas fonctionné et les remboursements se sont éternisés du fait d'un fort taux d'intérêt (9\%).

Parmi les causes externes de la faillite du FONADER, il convient de citer la crise économique des années 1980 liée à la dépréciation du dollar et à la chute des prix des produits d'exportation tels que le café, le cacao, le coton ou la banane. En effet, le FONADER est principalement financé par la Caisse de Stabilisation. Or, à partir de la fin des années 1970, les prix de ces produits de base ont entamé une baisse qui s'est prolongée jusqu'à la fin des années 1980. La situation est exacerbée par le retrait de la Banque mondiale qui constitue avec ses partenaires l'une des principales sources de financements du FONADER. Il en résulte un amenuisement des sources de financement $\mathrm{du}$ FONADER à la fin des années 1980.
L'autre facteur externe ayant entraîné la chute du FONADER est le désengagement de l'État des secteurs productifs consécutivement à la crise économique des années 1980. Ainsi, tous les organismes d'intervention de l'État dans l'économie et le monde rural sont fermés progressivement. Le FONADER qui est l'un des principaux moyens d'intervention de l'État dans le monde rural est aussi appelé à disparaître. La fin des années 1980 marque une rupture du partenariat entre les petits planteurs de palmiers à huile bénéficiaires d'un crédit de plantation et les agro-industries publiques en charge de son recouvrement. C'est l'un des principaux facteurs de la faillite du FONADER, et plusieurs raisons expliquent cette rupture.

\subsection{La rupture du partenariat entre agro-industries publiques et petits planteurs}

Avec la crise et l'essoufflement du FONADER à la fin des années 1980, les agro-industries publiques ne sont plus en mesure d'assurer l'entretien des pistes rurales. Pourtant, c'est l'une de leurs missions régaliennes compte tenu de leur statut de société de développement et au regard des clauses du contrat qui les lie aux planteurs dans le cadre du programme de plantations villageoises. Certaines pistes sont ainsi devenues impraticables surtout en saison pluvieuse. À cela, il faut ajouter la modification des termes de contrat par les agro-industries sans une consultation préalable des planteurs encadrés. En 1982, la CDC restreint la localisation des plantations non plus à proximité des routes carrossables, mais plutôt des routes goudronnées (Koenings, 1986). Ceci réduit la zone de collecte de l'agroindustrie et désavantage les exploitations situées hors de cette nouvelle zone instituée par la CDC. Cette situation entrave la collecte des régimes des plantations villageoises et leur acheminement vers les huileries comme le stipule le contrat surtout dans les localités enclavées. Les régimes des petits planteurs ne sont plus collectés régulièrement comme à l'accoutumée. Ces derniers subissent de nombreuses pertes qui ne sont d'ailleurs pas prises en compte par l'agro-industrie.

Un autre facteur explicatif de la rupture du partenariat agro-industries/petits planteurs 
concerne le prix d'achat des régimes livrés par les petits producteurs. Il n'est pas prévu dans le contrat que celui-ci change en fonction des cours mondiaux de l'huile de palme, mais les agro-industries publiques ont décidé de manière unilatérale de le modifier. Au début du programme, le régime provenant des plantations villageoises sous programme était acheté par la SOCAPALM à 27 francs CFA $/ \mathrm{kg}$. En 1990, ce prix était tombé à 20 francs $^{18}$. Les planteurs contestent ce prix imposé sans négociation et finissent par se convaincre qu'ils sont exploités par l'agro-industrie.

Une autre source de problème est liée au système de pesage des régimes désormais pratiqué par certaines agro-industries comme la SOCAPALM. Jusqu'en 1987, les planteurs ne trouvent pas de reproche au système de pesage de leurs régimes qui détermine leurs revenus. Le processus peut être décrit de la manière suivante : le camion vide est d'abord pesé puis chargé de régimes avant d'être pesé à nouveau. Ensuite, on fait la soustraction entre le poids du camion chargé et son poids à vide. Toutes ces opérations se font en présence du planteur qui a accès au pont bascule. Mais après cette date, ce n'est plus le cas. Le planteur n'a plus accès au pont bascule et le poids de ses régimes lui est simplement communiqué sur une fiche remplie à la main, ce qui n'inspire pas confiance.

En plus, les autres services d'encadrement et de fourniture d'engrais et de plants sélectionnés ne sont plus assurés par les agro-industries. Les services sociaux tels que la construction des écoles, la construction des centres de santé et l'électrification rurale dans les secteurs de plantations villageoises ne connaissent pas un meilleur sort. Cette situation est plus marquée dans la zone anglophone où les autochtones notamment les Bakwéri se plaignent de ne plus voir de différence de ce point de vue entre la zone anglophone et la zone francophone, alors que ces avantages sociaux font partie du contrat d'attribution des terres sous forme de concession à la CDC. En effet, la création même de la CDC répond d'abord à un souci de développement des chefs coutumiers qui acceptent que des concessions soient attribuées à cette entreprise contre la

18 Entretien avec un ancien bénéficiaire du projet de plantations villageoises à Makondo, dans la Sanaga-Maritime le 18 avril 2013. mise en place d'actions sanitaires, sociales et de développement.

Certains paysans se plaignent que le remboursement du crédit consenti a l'air d'être éternel. Ils ignorent tout de l'échéance du crédit car les agro-industries ne communiquent pas régulièrement le montant des encours aux petits planteurs.

Certains planteurs arguent enfin que l'assistance financière des agro-industries est insuffisante par rapport à leurs efforts consentis pour la culture, l'entretien et la récolte dans les plantations. Certains planteurs sont même parfois obligés de recruter de la main d'œuvre pour mener leurs activités. En retour, le revenu de la vente des régimes ne leur permet pas de couvrir leurs dépenses et de dégager une épargne suffisante pour leurs familles. Ils sont parfois contraints d'engager leur maigre épargne ou de contracter des crédits. Cette perception rejoint la position d'Elong (2008) lorsqu'il déclare : « quand on observe attentivement le revenu net annuel ou mensuel provenant d'un hectare de palmeraie villageoise, on constate que c'est à juste titre que les paysans disent que la culture du palmier à huile à base de plants sélectionnés ne rapporte pas beaucoup de profit. Pendant la première année de production, ce profit est de $2250 \mathrm{~F}$ CFA par mois et ce n'est qu’à la treizième année qu'il tourne autour de 10000 F CFA. Par conséquent, si cultiver les plants sélectionnés de palmier à huile exige tant d'efforts, de patience, pour aboutir à peu de profit, il y a lieu de comprendre le faible engouement de la population paysanne pour ce projet. " Cet auteur a certainement effectué son analyse économique à partir du modèle de remboursement des crédits de la Banque mondiale et d'un modèle de production local réaliste, montrant ainsi que les petits planteurs ne peuvent pas rembourser en 7 ans, un crédit à un tel taux à partir de la septième année. De nombreux auteurs montrent un impact important de la culture du palmier à huile sélectionné sur les revenus des paysans. Bakoumé et al. (2002) indiquent que "les petits élæiculteurs enregistrent une amélioration de leur statut par rapport à la moyenne de la communauté villageoise ». Hirsh (1999) pour sa part estime qu'un hectare de palmier sélectionné offre 167000 à 307000 francs CFA de revenus nets en année de croisière et en fonction du mode d'exploitation. 
En conclusion, les palmeraies sélectionnées sont très rentables et suscitent dès les années 1990 un engouement fort de toute la population rurale et urbaine, mais le modèle de remboursement de la Banque mondiale n'a pas été applicable du fait du taux d'intérêt élevé du crédit.

Finalement, le mauvais climat qui règne autour $\mathrm{du}$ partenariat entre les agro-industries et les petits planteurs amène ces derniers à développer deux trajectoires alternatives. Certains planteurs cessent de livrer leurs régimes aux agro-industries et décident de transformer eux-mêmes leurs régimes. Ils achètent des presses manuelles ou motorisées pour l'auto-transformation de leurs produits et de ceux de leurs voisins qui utilisent leur presse contre $20 \%$ de l'huile extraite. Lorsqu'un planteur ne parvient pas à traiter intégralement sa production, surtout en période de pointe, il continue d'en livrer une partie à l'agro-industrie. Une autre catégorie de petits planteurs opte pour une livraison des régimes à une autre huilerie industrielle concurrente, privée ou publique afin d'éviter de rembourser le crédit de plantation.

En résumé, la détérioration du partenariat entre les agro-industries et les planteurs dans le cadre du programme de plantations villageoises de palmier à huile n'est que partiellement imputable à la crise économique des années 1980. Celle-ci entraîne la chute du FONADER qui est la source de financement de ce programme. Cette situation place les agro-industries dans l'incapacité d'assurer leurs missions de sociétés de développement et de respecter les termes du contrat signé avec les planteurs. En plus, ces agro-industries n'associent pas suffisamment les planteurs dans la gestion de cette crise qui nécessite certainement une révision des contrats et une stratégie communicationnelle particulière. Finalement, les planteurs suspendent la livraison de leurs régimes aux agro-industries soit en livrant leurs régimes à une autre huilerie, soit en les traitant artisanalement ; ce qui leur permet d'échapper au remboursement du crédit FONADER. Cette crise est venue néanmoins réduire la dépendance des planteurs vis-à-vis des agro-industries. Désormais, plusieurs choix de débouchés s'offrent à eux pour l'écoulement de leurs productions. La fin des années 1980 ouvre ainsi une nouvelle ère dans le développement de la filière palmier à huile.

\subsection{Un modèle de développement élæicole réussi mais éphémère}

Au lendemain de la Deuxième Guerre mondiale, le Cameroun décide de moderniser sa filière élæicole avec l'aide des bailleurs de fonds internationaux. Le processus commence par la création de la station de la Dibamba qui permet désormais de disposer des plants sélectionnés de palmier à huile de grande qualité génétique dans le pays. La modernisation de la culture du palmier à huile se poursuit avec la mise en place des complexes agro-industriels et plus particulièrement de la SOCAPALM en 1968. À l'aide des financements du FONADER créé en 1973, l'État met en œuvre un vaste programme de plantations villageoises de palmier à huile entre 1979 et 1991 principalement par l'intermédiaire de la SOCAPALM et de la CDC. Ce programme est à la base d'un partenariat fructueux entre les petits producteurs ruraux et les agro-industries jusqu'à la liquidation du FONADER en 1991. Dès lors, la filière palmier à huile connaît de nombreuses mutations qui se poursuivent à l'heure actuelle. 


\section{La nouvelle organisation du secteur à partir de 1990}

\subsection{Le contexte sociopolitique du Cameroun au début des années 1990}

Le contexte socioéconomique et politique du Cameroun est marqué par la libéralisation économique, l'instauration de la démocratie et du multipartisme, ainsi que la définition et la mise en œuvre de la nouvelle politique agricole du Cameroun.

\subsubsection{La libéralisation économique et le désengagement de l'État}

La crise économique des années 1980 entraîne de profondes mutations dans l'agriculture camerounaise. Elle oblige le Cameroun à se tourner vers les institutions internationales afin de retrouver le chemin de la croissance économique. Dès 1988, plusieurs programmes de réforme économique sont signés avec le FMI et la Banque mondiale. Plusieurs mesures législatives sont prises pour asseoir les politiques de libéralisation. Dans le domaine des cultures pérennes, le libéralisme économique est lancé par la loi $n^{\circ} 95 / 11 \mathrm{du}$ 27 juillet 1991 portant organisation du commerce du cacao et du café et son décret d'application $\mathrm{n}^{\circ}$ 95/674/PM du 15 décembre 1995. Ces textes législatif et réglementaire constituent la base juridique de la libéralisation agricole des cultures pérennes au Cameroun et en fixent les cadres et les conditions d'exercice (Awumou, 2006). En outre, l'État instaure également une réglementation favorisant l'émergence d'organisation paysannes et communautaires. Il s'agit entre autres textes de :

- La loi nº 90/053 du 19 décembre 1990 sur la liberté d'association en remplacement de la loi $n^{\circ}$ 67/LF/19 du 12 juin 1967 qui elle-même abrogeait la loi de 1901 ;

- La loi nº 92/006 du 14 août 1992 sur les sociétés coopératives et les groupes d'initiatives communes (GIC), en remplacement de la loi $\mathrm{N}^{\circ} 73 / 15 \mathrm{du} 7$ décembre 1973 portant statut des sociétés coopératives.
Ces textes en permettant de légaliser et de structurer de nombreuses organisations paysannes forment la base de la restructuration des acteurs agricoles. Notons cependant que la souplesse de cette loi laisse la place à des dérives. Des sociétés privées de prestations de services, sans aucun rapport avec le monde rural et encore moins agricole, peuvent se légaliser en GIC pour échapper à la fiscalité (Awoumou, 2006). Ces réformes visent à instaurer la concurrence dans la commercialisation des produits agricoles et de responsabiliser les opérateurs privés.

Cette libéralisation de l'économie entraîne le désengagement de l'État dans les secteurs productifs, la privatisation et la liquidation voire la dissolution des sociétés publiques de production agricole sous l'instigation des institutions de Bretton Woods (la Banque mondiale et le FMI) et avec le concours du l'Organisation Mondiale du Commerce (OMC). C'est dans cette perspective que, dans la filière palmier à huile, la SOCAPALM est privatisée en 2000 tandis que la PAMOL et la CDC ne trouvant pas acquéreur restent sous tutelle de l'État. Malheureusement, on se rend compte que le secteur privé ne prend pas la relève de l'État, notamment en ce qui concerne l'octroi de crédits de plantation suite à l'expérience du FONADER. En revanche, l'approvisionnement en engrais est assuré avec des facilités de paiement uniquement pour les petits planteurs qui livrent des régimes tous les mois aux 3 agro-industries de la zone francophone qui sont concurrentes au cours des années 1990 pour l'achat des régimes villageois, tandis que les 2 agro-industries en quête d'investisseur privé de la zone anglophone qui manquent de trésorerie, ne peuvent pas se permettre de proposer un crédit de campagne aux petits planteurs de leur bassin d'approvisionnement. En l'absence de crédit de plantation, il est devenu extrêmement difficile pour les ruraux les plus démunis d'investir pour créer une palmeraie sélectionnée; ils se retrouvent abandonnés à eux-mêmes. Ceci n'a pas permis 
aux «gens de la brousse » (Ela J.M 1981 cité par Elong, 2011) de s'insérer dans les nouveaux enjeux économiques de la libéralisation, à cause de leurs faibles capacités à s'adapter aux réalités du marché national et international marquées par la compétitivité et la concurrence, et qui exigent d'eux, savoir-faire, moyens humains, financiers et matériels pas du tout en leur possession (Elong, 2011). On assiste donc à l'émergence de nouveaux acteurs notamment les élites urbaines et les migrants de retour dans la filière élæicole.

\subsubsection{L'instauration de la démocratie et la bataille de positionnement politique}

Sur le plan politique, les années 1990 sont également caractérisées par l'instauration du multipartisme. Il permet à certains acteurs notamment aux élites de s'insérer dans les jeux démocratiques qui facilitent la construction politique et économique. Le multipartisme est également à l'origine d'une bataille de positionnement politique entre les acteurs politiques qui veulent s'imposer dans les cercles locaux de prise de décision. Cette projection a un impact certain sur le monde agricole camerounais car en Afrique, et au Cameroun particulièrement, le positionnement politique n'a une signification réelle que lorsqu'il est ancré dans la région et surtout dans le village d'origine (Elong, 2011). Pour conquérir l'électorat des producteurs ruraux en faveur de leurs partis politiques, les élites urbaines ont souvent l'habitude d'investir dans le secteur agricole et plus particulièrement dans la filière palmier à huile, en créant des palmeraies dans leur village d'origine. C'est dans ce contexte sociopolitique marqué par la libéralisation et le multipartisme que la Nouvelle Politique Agricole du Cameroun (NPAC) est élaborée en 1990.

\subsubsection{La mise en place de la nouvelle politique agricole du Cameroun}

La Nouvelle Politique Agricole du Cameroun (encadré 3) cherche à consolider les acquis de l'agriculture camerounaise et à améliorer les performances enregistrées. En résumé, dans le cadre de la NPA, l'État s'efforce de créer un cadre stratégique favorable à l'initiative privée et d'édicter des mesures de déréglementation et de privatisation visant à réduire les gaspillages, à rationaliser les ressources et à trouver des modes de gestion plus efficients. La privatisation de la gestion du capital des entreprises parapubliques vise à réduire les effets d'éviction du secteur public. Les entreprises inefficientes sont liquidées et celles qui connaissent des dysfonctionnements réhabilitées (Ondoa Manga, 2006).

Dans la filière huile de palme, les trois unités de production publiques (SOCAPALM, PAMOL et $\mathrm{CDC}$ ) ainsi que les deux privées (SAFACAM et SPFS) sont autorisées à créer des usines de raffinage de l'huile brute, afin d'augmenter leur marge en créant une valeur ajoutée par la transformation de l'huile, au moment où les cours internationaux sont bas.

Au début des années 1990, la filière palmier à huile est donc sujette à de multiples recompositions sociospatiales qui affectent les systèmes d'exploitation. Les différents acteurs s'organisent pendant que l'interventionnisme de l'État refait surface à travers de nombreux projets. Ces recompositions sociospatiales ouvrent de nouvelles perspectives de développement du palmier à huile au Cameroun.

\subsection{Les principaux systèmes de production d'huile de palme au Cameroun}

Jusqu'à la fin des années 1980, le développement de la culture du palmier à huile au Cameroun est dominé par les agro-industries. Avec le début de la décennie 1990, les acteurs intervenant dans la culture du palmier se diversifient. On distingue essentiellement deux catégories d'acteurs plus ou moins indépendants, à savoir les agroindustries qui exploitent 60000 ha de palmeraies industrielles et les planteurs villageois qui gèrent environ 40000 ha de palmeraies sélectionnées et des surfaces non estimées de plantations de palmier tout-venant et de palmeraies dites "naturelles " encore exploitées dans les zones où il n'y a pas eu d'action de développement de l'élaeiculture (Bakoumé et al. 2002).

\subsubsection{Un système agro-industriel en crise}

Jusqu'en 2001, il existe 5 agro-industries au Cameroun. Elles sont toutes implantées dans 


\section{Encadré 3 : Les objectifs de la Nouvelle Politique Agricole du Cameroun}

La Nouvelle Politique Agricole du Cameroun poursuit 5 principaux objectifs à savoir :

\section{1. la modernisation de l'appareil de production}

À travers elle, le matériel végétal amélioré doit être rendu plus disponible et accessible aux producteurs grâce à la privatisation de sa production et de sa distribution ; la privatisation de l'importation et de la distribution des engrais et pesticides vont se poursuivre ; le Centre d'Étude et d'Expérimentation du Machinisme Agricole (CENEEMA) doit être transformé en un organisme performant de prestation de service mettant des machines et outils agricoles adaptés à la disposition des agriculteurs ; un programme de «formation et visites » négocié avec la Banque mondiale doit permettre d'améliorer les méthodes d'intervention des services de vulgarisation traditionnels qui se verront en outre transférer les missions d'encadrement des sociétés de développement ; l'État se désengage de la gestion des coopératives qui doivent désormais se créer et se gérer librement par les agriculteurs autour d'objectifs économiques précis (acquisition d'intrants, commercialisation des produits, crédits agricoles...), le Crédit Agricole en création doit devenir l'instrument principal de la modernisation de l'agriculture et de la promotion des exploitations agricoles de moyenne importance ; une libéralisation progressive de la commercialisation interne et externe des produits agricoles pour en accroitre la compétitivité.

\section{2. la sécurité alimentaire}

À travers cet objectif, la relative autosuffisance alimentaire doit être traduite en sécurité alimentaire. Un accent mis au niveau de la production agricole sur la diffusion des résultats de la recherche agronomique ; l'intensification de la recherche sur des variétés adaptées ; une spécialisation des régions dans la production afin d'exploiter au mieux les potentialités diverses ; la création de marchés frontaliers afin de profiter des débouchés que constituent les pays voisins. L'amélioration de la conservation des denrées dont il est estimé que 15 à $20 \%$ des céréales et 30 à $40 \%$ des tubercules sont perdus faute d'une conservation adéquate ; l'organisation du commerce inter-régional et l'amélioration des infrastructures de communication et de commercialisation ; le lancement d'un Fonds d'Investissement de Microréalisations Agricoles et Communautaire (FIMAC) pour créer des emplois et des revenus additionnels en vue d'acquérir des denrées alimentaires et plus globalement la préparation d'un programme de sécurité alimentaire avec l'appui de la Banque mondiale.

\section{3. la promotion et la diversification des exportations}

Elle a pour objet d'améliorer les recettes d'exportation, suite aux difficultés rencontrées par les produits traditionnels d'exportation. À cet effet, des études doivent être conduites pour prospecter des marchés extérieurs porteurs, y compris les marchés des pays voisins et pour proposer des projets d'investissement et d'organisation de la production et de la commercialisation.

\section{4. le développement de la transformation des produits agricoles}

La zone franche qui vient d'être créée au Cameroun doit aider à la promotion des unités de transformation des produits agricoles qui sont jusque-là exportés sous forme brute de manière à créer une valeur ajoutée. Pour ce faire, le point des technologies de transformation existantes dans le pays doit être fait et des études de rentabilité et d'exploitabilité réalisées.

\section{I'équilibre des filières de production}

Dans le cadre de cet objectif, chaque grande culture doit faire l'objet d'un ensemble de mesures de relance. Dans la filière riz, la réhabilitation des unités de production et la mise en œuvre d'une caisse de péréquation doivent permettre de valoriser le potentiel de production 70000 tonnes de riz produites par le secteur moderne local alors que la consommation nationale est estimée à plus du double de ce tonnage 
la zone côtière du pays où les conditions agroécologiques sont optimales. Parmi ces opérateurs, deux entreprises (PAMOL et CDC) sont publiques tandis que les trois restantes (SOCAPALM, SAFACAM et SPFS) sont privées et appartiennent au groupe Bolloré.

\section{La Société Camerounaise de Palmeraies (SOCAPALM)}

C'est l'acteur majeur de la filière palmier à huile au Cameroun. Créée en 1968 sous forme de Société publique de développement, elle a été privatisée en 2000. Suite à cette opération, la SOCAPALM obtient une concession de terres de 78029 ha pour une durée de 60 ans. Cette superficie est ramenée à 58063 ha par un avenant conclu en 2005. La SOCAPALM possède 5 plantations localisées à Mbongo, Mbambou, Eséka, Dibombari, et Kienké. Pour trouver une solution définitive aux vols de régimes dans ses palmeraies vieillissantes de Dibombari et afin de pouvoir traiter dans son huilerie la production de régimes villageois produite au nord de Dibombari, la SOCAPALM replante ses vieilles palmeraies avec de l'hévéa. La superficie des plantations d'hévéas sur le site de Dibombari sont étendues à environ 1500 ha en 2009 (SOCAPALM, 2009).

\section{La Cameroon Development Corporation (CDC)}

La CDC est une Société publique de développement créée après les indépendances pour succéder à la Commonwealth Development Corporation (COMDEV) créée en $1947 \mathrm{au}$ lendemain de la Seconde Guerre mondiale dans le Cameroun sous tutelle britannique. En dehors du palmier à huile, la CDC gère des plantations d'hévéa, de bananier, de théier et de poivrier.

\section{La Société des Palmeraies de la Ferme Suisse (SPFS - PALM'OR)}

Elle est située à Apouh près d'Edéa sur la route de Kribi. La SPFS a une concession de 5700 ha sur laquelle elle produit des régimes qu'elle transforme dans sa propre huilerie en huile brute puis en huile raffinée dans sa propre raffinerie. L'huilerie de la SPFS s'approvisionne à l'extérieur en achetant des régimes villageois, tandis que sa raffinerie traite de l'huile brute de la SAFACAM et de la SOCAPALM. La SPFS s'est également lancée dans la production de di-ester qu'elle utilise comme agro-carburant pour ses propres besoins énergétiques. Depuis 2001, la SPFS est devenue une filiale de la SOCAPALM.

\section{La Société Africaine Forestière et Agricole du Cameroun (SAFACAM)}

La SAFACAM est située à Dizangué non loin d'Edéa. C'est une société privée créée en 1897 au capital 2070000000 FCFA. Elle dispose d'une concession de 15500 hectares dont 4700 hectares en palmier à huile depuis 1974. En plus de ses palmeraies industrielles et de son huilerie de $10 \mathrm{t} / \mathrm{h}$, elle a aussi des plantations d'hévéa et une unité de traitement. En 2013, ses palmeraies couvrent une superficie de 3700 hectares en rapport, 900 hectares immatures, tandis que 100 hectares de la concession ne sont pas encore mis en valeur. Elle produit de l'huile de palme brute.

\section{La PAMOL}

La PAMOL est une Société parapublique depuis 1980 créée avec un capital de 1800000000 FCFA reparti entre l'État (54\%), UNILEVER (10\%) et les $36 \%$ restants sont détenus par la Caisse Nationale de Prévoyance Sociale (CNPS), SOCAPALM, CDC, SAFACAM, et SPFS (PACA, 2009).

En 2009, les agro-industries produisent plus de $145000 \mathrm{t}$ d'huile de palme brute sur une superficie globale de 60784 ha de palmeraies (Tableau 5), avec des rendements en régimes dans les différentes plantations industrielles compris entre 5 et $20 \mathrm{t} / \mathrm{ha}$, selon les conditions pédoclimatiques, le matériel végétal, les pratiques et problèmes de vols. Ceci est très insuffisant compte tenu des conditions agroécologiques dont bénéficient les plantations agroindustrielles et du potentiel estimé entre 15 et $20 \mathrm{t}$

Tableau 5 : Production d'huile de palme par les agro-industries au Cameroun en 2008

\begin{tabular}{lll}
\hline Société & \multicolumn{1}{c}{$\begin{array}{c}\text { Surface totale } \\
(\mathbf{h a})\end{array}$} & $\begin{array}{c}\text { Huile brute } \\
(\mathbf{t})\end{array}$ \\
\hline SOCAPALM & 27773 & 83000 \\
\hline SPFS & 3228 & 15000 \\
\hline SAFACAM & 4633 & 12000 \\
\hline CDC & 15607 & 18000 \\
\hline PAMOL & 9543 & 16000 \\
\hline TOTAL & 60784 & 145000 \\
\hline
\end{tabular}

Source: Lebailly et al., 2009

NB : L'huile brute est produite à partir des régimes produits dans les plantations industrielles et de régimes villageois achetés aux petits planteurs. 
de régimes à l'hectare pour les matériels végétaux de second et de troisième cycle de sélection mis à leur disposition (Bakoumé et al., 2002).

Avec presque la moitié des plantations industrielles et plus de la moitié de la production d'huile brute, la SOCAPALM est l'agro-industriel majeur du Cameroun, loin devant des agro-industries comme la CDC, la PAMOL et la SAFACAM. La part de la production consolidée de la SOCAPALM et de la SPFS est de 67,7 \% (SOCAPALM, 2009).

\subsubsection{Un secteur villageois en pleine expansion}

Le secteur villageois est animé par des acteurs appelés communément petits planteurs à cause du caractère réduit des surfaces mises en valeur comparativement à celles des agro-industries. De manière générale, on distingue deux types de petits planteurs de palmier à huile au Cameroun depuis le début des années 1990, à savoir les petits planteurs encadrés par les agro-industries auxquelles ils livrent au moins une partie de leurs régimes et les petits planteurs indépendants dont $100 \%$ de la production sont traités artisanalement par le petit planteur, ou par un tiers auquel il vend ses régimes et ses fruits détachés.

Les petits planteurs encadrés ont leurs exploitations situées dans les bassins d'approvisionnement des unités agro-industrielles avec lesquelles ils ont établi une relation commerciale. Certains ont commencé à planter leurs premières parcelles de palmiers en bénéficiant du crédit de plantation octroyé entre 1978 et 1991 par le FONADER. Ceux qui étaient en dehors de la zone ou de la période d'intervention du projet ont commencé à planter sans soutien financier. La superficie moyenne de ces plantations est de 5 ha par planteur tandis que la majorité des surfaces varie entre 2 et 3 ha (Bakoumé et al., 2002). En 1993, lors de la définition du plan de relance du secteur agro-industriel connu sous le nom de Table Ronde de Limbé, 3178 planteurs sont enregistrés auprès d'une agro-industrie à laquelle ils livrent plus ou moins régulièrement des régimes (Tableau 6 ).

Grâce au crédit de plantation du FONADER, ces petits planteurs créent plus de 14427 ha de palmeraies sélectionnées situées essentiellement dans les départements de la Sanaga-Maritime, du Nyong-et-Kéllé, du Ndian et du Fako.

En 2008, l'UNEXPALM, une organisation professionnelle, évalue la superficie des plantations villageoises de petits planteurs encadrées à plus de 35000 ha et dénombre plus de 10000 planteurs. Dans 5 sites, le MINADER évalue la superficie des plantations villageoises des petits planteurs encadrés à plus de 11686 ha, dont 9132 en production (tableau 7).

Le rendement apparent est calculé à partir des volumes livrés à l'huilerie et des surfaces déclarées, sachant que parfois des voisins non enregistrés sollicitent occasionnellement un planteur enregistré pour ajouter des régimes au chargement, que les inventaires de surfaces sont rarement mis à jour par

Tableau 6 : Les petits planteurs encadrés en 1993

\begin{tabular}{lccc}
\hline Secteur « villageois » des agro-industries & Nombre de planteurs & Surfaces (ha) & Superficie/planteur \\
\hline SOCAPALM Dibombari & 824 & 3894 & 4,7 \\
\hline SOCAPALM Edéa & 843 & 2489 & 2,9 \\
\hline SOCAPALM Eséka & 727 & 1814 & 2,5 \\
\hline PAMOL Ekondo-Titi & 351 & 2508 & 7,1 \\
\hline PAMOL Mundemba & 162 & 1599 & 9,9 \\
\hline CDC & 193 & 699 & 3,6 \\
\hline SAFACAM Dizangue & 13 & 417 & 32,1 \\
\hline S.P.F.S. Edéa & 60 & 1007 & 16,8 \\
\hline Total & $\mathbf{3 1 7 8}$ & $\mathbf{1 4 4 2 7}$ & $\mathbf{4 , 5}$ \\
\hline
\end{tabular}


Tableau 7 : Caractéristiques de quelques sites de plantations encadrées en 2012

\begin{tabular}{lcccc}
\hline Sites & $\begin{array}{c}\text { Nbre de } \\
\text { planteurs }\end{array}$ & $\begin{array}{c}\text { Rendement } \\
\text { Moyen apparent }\end{array}$ & $\begin{array}{c}\text { Superficie en } \\
\text { production (ha) }\end{array}$ & $\begin{array}{c}\text { Superficie non en } \\
\text { production (ha) }\end{array}$ \\
\hline SOCAPALM Edéa & 238 & $8 \mathrm{t} / \mathrm{ha} / \mathrm{an}$ & 784 & 324 \\
\hline SOCAPALM Mbongo & 335 & $8 \mathrm{t} / \mathrm{ha} / \mathrm{an}$ & 4608 & 1386 \\
\hline SOCAPALM Eséka & 682 & $7 \mathrm{t} / \mathrm{ha} / \mathrm{an}$ & 1619 & 421 \\
\hline FAKO & 447 & $8 \mathrm{t} / \mathrm{ha} / \mathrm{an}$ & 1695 & 342 \\
\hline NDJOCKLOUMBE & 53 & $8 \mathrm{t} / \mathrm{ha} / \mathrm{an}$ & 425 & 80 \\
\hline TOTAL & 1755 & $7,8 \mathrm{t} / \mathrm{ha} / \mathrm{an}$ & 9132 & 2554 \\
\hline Total des superficies des plantations villageoises & & 11686 &
\end{tabular}

Source : MINADER, 2012

les huileries et que les planteurs ne connaissent pas tous leurs surfaces réellement plantées.

Les petits planteurs encadrés ont un rendement apparent moyen de 7,8 $\mathrm{t}$ de régimes/ha/an (MINADER, 2012), soit moins de $2 \mathrm{t} / \mathrm{ha}$ d'huile de palme brute. Des enquêtes menées chez des petits planteurs encadrés et indépendants estiment un rendement moyen en régimes à $6 \mathrm{t} /$ ha (Bakoumé, 2002), ce qui semble cohérent avec l'estimation du MINADER du fait d'une proportion plus élevée de matériel tout venant chez les petits planteurs indépendants. Hoyle et Levang (2012) évaluent la production d'huile de palme brute à $30000 \mathrm{t}$ à partir des régimes livrés par les petits planteurs encadrés, pour une superficie estimée à 35000 ha, ce qui correspond à un rendement apparent de $0,9 \mathrm{t}$ d'huile par ha. Dans cette dernière estimation, la part de la production qui est transformée artisanalement par les petits planteurs encadrés n'est pas intégrée, ce qui explique le facteur 2 avec les estimations précédentes. En effet, les fruits détachés qui contiennent $50 \%$ d'huile, sont rarement livrés à l'huilerie, tandis qu'une partie des régimes produits sur les 35000 ha est transformée artisanalement.

Après la liquidation du FONADER en 1991, le développement des palmeraies villageoises se poursuit de manière spontanée, sans crédits de plantation ni d'appuis techniques publics, aussi bien dans les bassins d'approvisionnement des agro-industries qu'à l'extérieur. En l'absence d'un recensement exhaustif de ces plantations, il est difficile de disposer de données fiables sur cette dynamique de plantation. Néanmoins, à partir de données de vente des graines sélectionnées aux petits planteurs des 2 producteurs nationaux, la station de l'IRAD à La Dibamba et la PAMOL à Lobé, Bakoume et al. (2002) évaluent la superficie des palmeraies sélectionnées plantées entre 1996 et 2001 à 28862 ha alors que les agro-industries ne plantent que 9267 ha au cours de la période. Ces chiffres, qui ne tiennent pas compte des superficies de palmeraies tout-venant du fait qu'elles ne sont pas estimables par la même méthode, indiquent que depuis 1996, la dynamique élæicole est portée par les petits planteurs. En effet, ces derniers plantent en moyenne $4800 \mathrm{ha} / \mathrm{an}$ d'extension entre 1996 et 2001 alors que les agro-industries ne plantent que 1500 ha/an d'extension et de replantation (fig. 12). La taille des concessions agro-industrielles limite leur capacité d'extension de surface.

Parmi les petits planteurs, les élites urbaines et rurales jouent un rôle de premier plan dans cette dynamique spontanée de plantation de palmeraies villageoises. Il s'agit pour l'essentiel de cadres supérieurs des secteurs public et privé, d'hommes politiques résidant en ville ou à l'étranger et possédant des revenus élevés et une grande influence politique, économique et sociale dans le pays ainsi que dans leurs villages et arrondissements d'origine. Le positionnement politique à proximité de leur électorat, la constitution des réserves foncières, la préparation de la retraite constituent les principales motivations de cette introduction des élites urbaines et rurales dans l'élæiculture. Il ne faut pas non plus négliger les migrants de retour ${ }^{19}$ qui créent généralement des palmeraies

19 Il s'agit des urbains qui rentrent dans leur villages d'origine pour des raisons diverses. La migration de retour est 


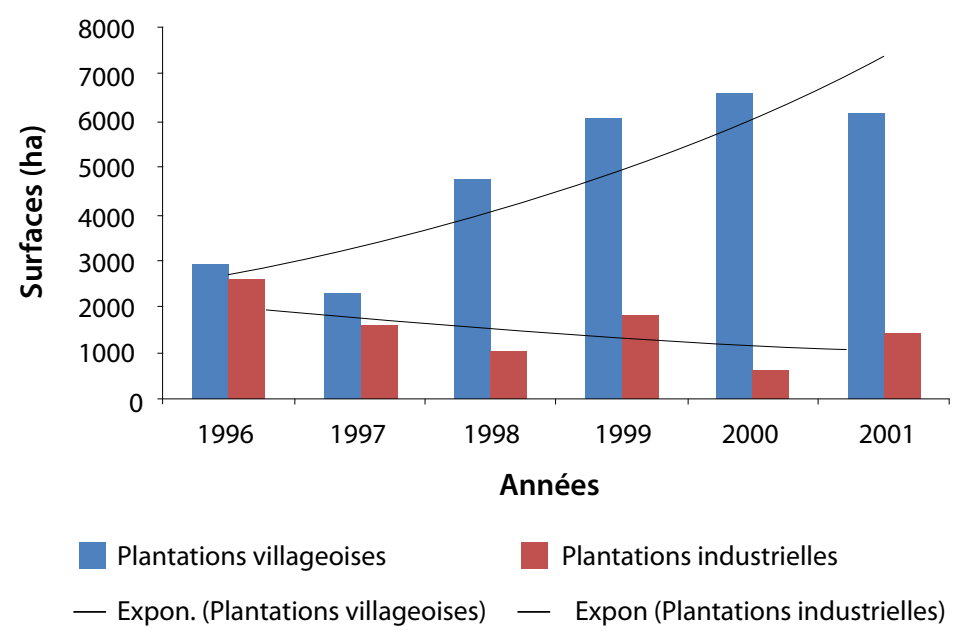

Figure 12 : Superficies des palmeraies sélectionnées plantées par les petits planteurs et les agro-industries du Cameroun entre 1996 et $2001^{\text {a }}$

Source : Bakoumé et al., 2002.

a Expon. renvoie à la courbe de tendance exponentielle.

Palm oil in Cameroon from 1964 to 2012

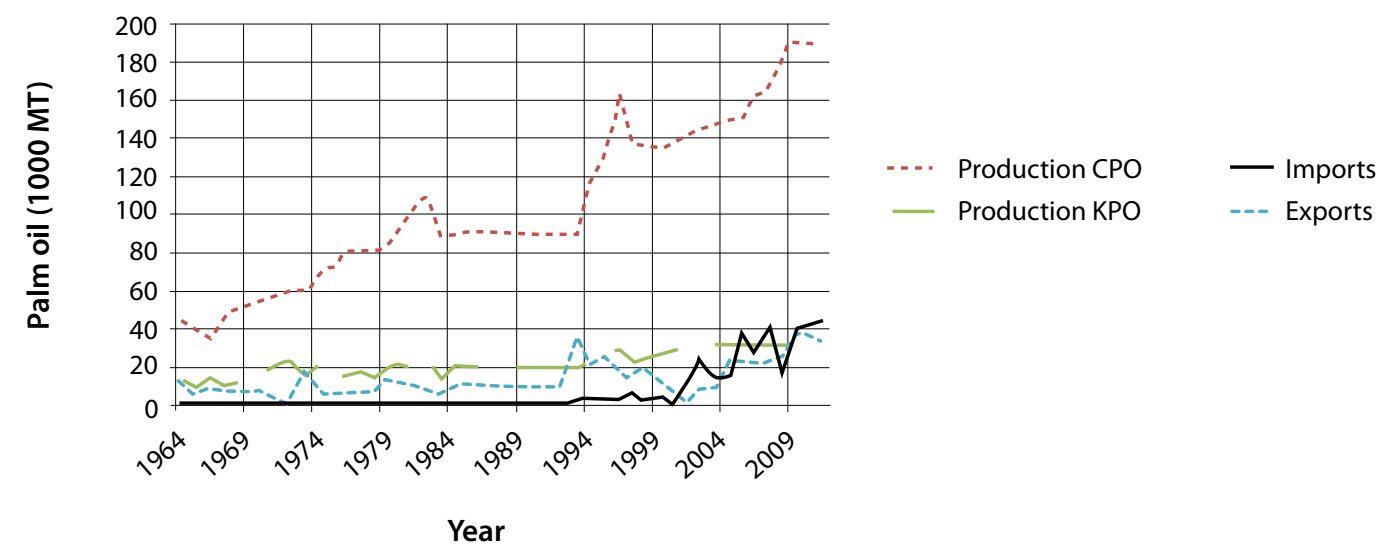

Figure 13 : Production d'huile de palme au Cameroun entre 1964 et 2012

Source : Feintrenie, 2012 d'après des données de http://www.indexmundi.com

de moindre envergure par rapport à celles des élites urbaines. Toutefois, comme il s'agit d'un développement spontané, sans crédit de plantation, chacun dimensionne son projet à ses moyens. Grâce à l'arrivée des élites qui implantent de grandes superficies, mais aussi à l'entrée en scène de la SAFACAM et de la SPFS qui vendent des plants sélectionnés de qualité aux petits planteurs

le contre-courant de l'exode rural. Ce phénomène apparaît et prend de l'ampleur au Cameroun au début des années 90 marqué par la mise en œuvre des plans d'ajustement structurel, du désengagement de l'État des secteurs productifs et de la privatisation des sociétés publiques et parapubliques. contre des régimes des palmeraies villageoises pour compléter l'approvisionnement de leurs huileries, la production d'huile de palme brute connaît une ascension fulgurante à partir de 1994 (fig. 13), au fur et à mesure que ces nouvelles extensions entrent en production.

En 2013, le MINADER évalue les superficies plantées de palmiers à huile au Cameroun à 210000 ha dont 70000 ha pour les agroindustries et 140000 ha pour les palmeraies villageoises (Ngom, 2013). Il s'agit d'une évaluation par recensement auprès des agro- 
Tableau 8 : Les principales huileries du Cameroun

\begin{tabular}{lcccc}
\hline Sociétés/Huileries & $\begin{array}{c}\text { Installation/ } \\
\text { extension }\end{array}$ & $\begin{array}{c}\text { Tonnage } \\
\text { moyen(t)/an }\end{array}$ & $\begin{array}{c}\text { Capacité } \\
\text { horaire (t/h) }\end{array}$ & $\begin{array}{c}\text { Production } \\
\text { CPO (t) }\end{array}$ \\
\hline CDC/Idenau & 1985 & 35000 & 25 & 7389 \\
\hline CDC/Mondoni & $1971 / 1974$ & 55546 & 25 & 11715 \\
\hline PAMOL/Lobe & $1965 / 1992$ & 62637 & 20 & 11757 \\
\hline PAMOL/Ndian & 1935 & $21300^{*}$ & 15 & N.C. \\
\hline SPFS/Apouh-Edéa & $1984 / 1992$ & 45695 & 10 & 9780 \\
\hline SAFACAM/Dizangue & 1980 & 45786 & 20 & 9441 \\
\hline SOCAPALM/Nkapa & $1978 / 1985$ & 86837 & 40 & 18279 \\
\hline SOCAPALM/Eséka & $1973 / 1974$ & 32834 & 10 & 6247 \\
\hline SOCAPALM/Kienke & $1982 / 1989$ & 65421 & 40 & 15035 \\
\hline SOCAPALM/Mbongo & $1974 / 1982$ & 80591 & 40 & 17765 \\
\hline Total & $\mathbf{5}$ sociétés et 10 huileries & $\mathbf{5 1 0 3 4 7}$ & $\mathbf{2 4 5}$ & $\mathbf{1 0 7 4 0 8}$ \\
\hline
\end{tabular}

Source : Hirsch, 1999; Bakoumé, 2002, Lebailly et al. 2009 *Données de 2001

industries pour les plantations industrielles et les palmeraies villageoises encadrées, tandis que pour les palmeraies villageoises indépendantes, c'est une évaluation par recensement auprès des techniciens vulgarisateurs, et par analyse d'images aériennes. Les palmeraies villageoises couvrent principalement la zone côtière du Cameroun, et la proportion de palmeraies sélectionnées et tout-venant reste une grande inconnue.

La production d'huile de palme est évaluée à $210000 \mathrm{t} / \mathrm{an}$ dont $140000 \mathrm{t} / \mathrm{an}$ d'huile brute produite par les agro-industries à partir de régimes industriels et villageois, et 70000 t/an d'huile rouge artisanale produite avec des régimes des palmeraies villageoises. Cependant, la balance des importations et exportations montre que le déficit intérieur en huile de palme est estimé à 50000 t en 2012 contre 85000 t en 2013 (Ngom, 2013) ${ }^{20}$.

\subsection{La première transformation des régimes de palme}

La première transformation des régimes de palmes issus des palmeraies industrielles s'effectue systématiquement dans une huilerie (sauf en cas de vol de régimes). Pour les régimes des palmeraies villageoises, on observe 2 cas :

20 Communication lors d'une réunion du Groupe de travail sur le développement durable du palmier à huile au Cameroun, à Yaoundé le 30 septembre 2013.
- dans les bassins d'approvisionnement des huileries, une part des régimes est traitée industriellement et l'autre artisanalement, avec des fluctuations saisonnières ;

- hors des bassins d'approvisionnement des huileries, tous les régimes sont traités artisanalement.

\subsubsection{Les huileries}

Actuellement 10 huileries industrielles fonctionnent au Cameroun avec une capacité de transformation de $245 \mathrm{t} / \mathrm{h}$. Bakoumé $e t$ al. estiment en 2002 que les volumes moyens traités à cette époque dépassent les 500000 t/ an de régimes pour une production annuelle de plus de 107000 tonnes d'huile de palme brute (Tableau 8). Leur capacité théorique annuelle est estimé à environ $60 \%$ tandis que les taux d'extraction avoisinent $21 \%$ pour l'huile de palme et $4 \%$ pour les palmistes (Bakoumé et al., 2002). La SOCAPALM a un projet de construction d'une autre usine à Mbambou non loin de Dizanguè dans la Sanaga-Maritime. Par ailleurs, il existe quelques minihuileries et microhuileries privées installées. On enregistre ainsi trois minihuileries de $9 \mathrm{t} / \mathrm{h}$ de régimes chacune dans le département du Dja-et-Lobo en 2008. Citons aussi l'unité d'extraction semi-artisanale d'huile de palme brute d'une capacité de 60 t de régimes par jour installée à Edéa Sud par la société DK OLMULEN (PACA, 2009). 


\subsubsection{Un développement sans précédent des presses artisanales}

La liquidation du FONADER a sonné la rupture du partenariat entre le secteur villageois et les agroindustries qui obligeait les petits planteurs à livrer leurs régimes aux huileries des sociétés publiques de développement. Depuis lors, des petits planteurs implantés dans les bassins d'approvisionnement des huileries effectuent le choix stratégique de transformer eux-mêmes l'intégralité ou une partie de leurs régimes. L'avènement des presses artisanales est la clé du développement élæicole en dehors des bassins d'approvisionnement des huileries. Ceci donne lieu à une profusion de presses artisanales dans et en dehors des principaux bassins de production d'huile de palme du pays notamment dans le Sud-Ouest, le Moungo, la Sanaga-Maritime et le Nyong-et-Kéllé. Elle contribue à l'augmentation de la production nationale d'huile de palme (figure 15).

Pendant la période de pic de production, les plantations villageoises situées à proximité des agro-industries préfèrent livrer leurs régimes en totalité ou en partie aux agro-industries, principalement à cause de l'indisponibilité de la main d'œuvre et des bas prix de vente de l'huile de palme pendant cette période. Pour les palmeraies éloignées des huileries industrielles, l'acquisition d'une presse ou bien la possibilité d'en utiliser une près de la palmeraie est indispensable. Ainsi, le développement élæicole loin des huileries grâce aux presses artisanales s'effectue en collaboration avec 2 nouvelles catégories d'acteurs qui ne sont pas des petits planteurs :
Tableau 9: La cession d'huile de palme brute aux transformateurs (2004-2007)

\begin{tabular}{cc}
\hline Années & Tonnage $(\mathbf{t})$ \\
\hline 2004 & 77450 \\
\hline 2005 & 94853 \\
\hline 2006 & 101509 \\
\hline 2007 & 96885 \\
\hline
\end{tabular}

Source : MINADER sur la base des données du SNPHPCa, 2012

a Syndicat des Producteurs d'Huile de Palme du Cameroun

- un premier groupe d'acteurs qui achète des régimes ou des fruits avant de les transformer avec leur propre presse ou bien une presse en location ;

- une deuxième catégorie regroupe des individus qui disposent de presses artisanales qu'ils louent aux propriétaires de palmeraies dépourvus d'unités de transformation de leurs régimes ou bien aux acheteurs de régimes.

Le PDPV, en collaboration avec l'UNEXPALM, a recensé 407 presses artisanales dans trois arrondissements de la Sanaga-Maritime (Edéa 1, Edéa 2 et Dibamba) en 2012. Sur les 407 presses, 98 sont motorisées tandis que 309 sont manuelles. Cependant, ces chiffres sous-estiment la situation réelle sur le terrain, car comme on peut le constater, ils portent essentiellement sur les plantations suivies par le programme.

Le taux d'extraction des presses artisanales oscille entre 12 et $18 \%$ comparativement à $21 \%$ pour les usines industrielles installées. De ce fait, une

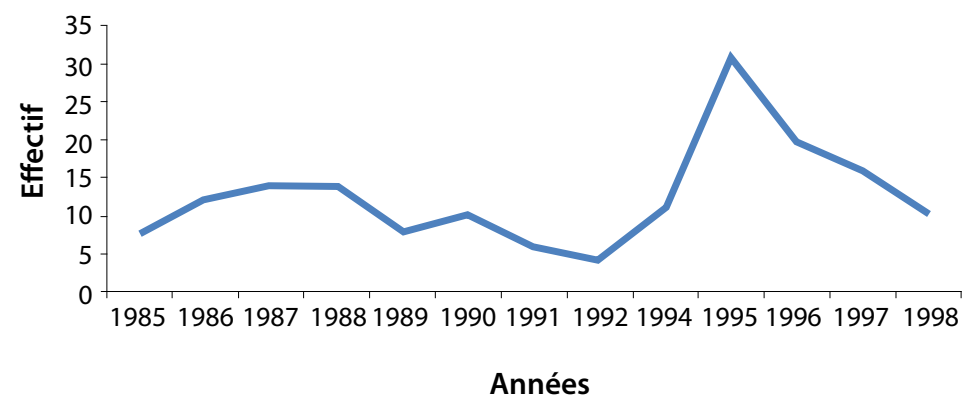

Figure 14: Vente des pressoirs artisanaux et production d'huile de palme au Cameroun Hirsch, 1999 
tonne de régime tenera traitée artisanalement permet d'obtenir entre 120 et $180 \mathrm{~kg}$ d'huile de palme alors qu'elle produit $210 \mathrm{~kg}$ d'huile de palme et $20 \mathrm{~kg}$ d'huile de palmiste lorsqu'elle est transformée par une huilerie (Bakoumé $e t$ al., 2002). Le matériel végétal tout-venant qui est très répandu loin des huileries, donc là où la transformation artisanale est obligatoire, accentue encore ces mauvaises performances de l'extraction artisanale.

La faible performance de l'extraction artisanale est l'argument le plus fréquemment avancé par les agro-industriels pour demander la disparition de ces unités concurrentes, accusées également d'être à l'origine du vol de régimes dans les palmeraies industrielles. Toutefois, le volume d'huile rouge artisanale produit est loin d'être négligeable et pourrait atteindre près de 30000 tonnes d'huile rouge artisanale par an (PACA, 2009).

\subsection{L'essor de la seconde transformation}

La seconde transformation regroupe les activités permettant d'élaborer une gamme variée de produits à base d'huile de palme ou de palmiste. Elles intéressent principalement les savonneries, les raffineries et la fabrication de la margarine. Le Cameroun compte plus d'une douzaine d'industries de seconde transformation d'huile de palme et de palmistes.

Les principales sont SCR-MAYA, CCC, CCIC, NOSA, SCS, ISF, SOC, GIRAFE, S.MIDI, ICC, SPFS-Palm'or, AZUR S.A., et les laboratoires BIOPHARMA. Plus de 50\% de ces entreprises sont localisées à Douala et ont été créées à partir des années 1990. Elles mettent sur le marché des produits aussi divers que des savons, des détergents, de l'huile raffinée, des cosmétiques, de la glycérine, des bougies, du cirage, principalement à destination du marché national et régional. Ces entreprises s'approvisionnent essentiellement auprès des producteurs nationaux. La demande en huile de palme brute des industries de seconde transformation ne cesse de croître (Tab. 16)

L'offre nationale en huile de palme brute reste insuffisante pour satisfaire les besoins de ces entreprises qui traitent les mêmes volumes par jour toute l'année. Comme la production d'huile de palme ne se distribue pas de manière homogène toute l'année, certaines entreprises de seconde transformation ont ainsi recours aux importations, lorsqu'en fin de saison de basse production, les stocks d'huile brute sont épuisés. C'est le cas de CCC qui s'approvisionne auprès de SIATGabon (MINADER, 2012) et de NOSA qui importe l'huile depuis la Malaisie (www.wrm.org. uy). Les plantations villageoises approvisionnent directement ou par des intermédiaires plusieurs de ces industries, à l'instar d'AZUR, qui transforment l'huile rouge artisanale en savon et en huile raffinée.

L'industrie de seconde transformation souffre de l'insuffisance de l'offre nationale en matières premières, notamment d'huile de palme brute et de palmistes à cause du déficit annuel de production estimé à 85000 t en 2013 contre 50000 t en 2012 (Ngom, 2013) ${ }^{21}$. La prolifération des industries de seconde transformation est en partie responsable de cette situation puisque leurs besoins augmentent plus vite que l'offre nationale. Le dynamisme de ce secteur doit néanmoins être perçu comme une opportunité de croissance de la filière car il stimule la production nationale d'huile de palme brute et de palmistes. Cependant, l'industrie de la seconde transformation est fortement menacée par la concurrence induite par l'importation des huiles raffinées sur le marché national par des négociants.

\subsection{L'organisation de la filière palmier à huile}

Depuis le début des années 1990, on note la création de quatre principales organisations professionnelles dans la filière palmier à huile au Cameroun.

\section{Le Syndicat National des Producteurs d'Huile de Palme du Cameroun (SNPHPC)}

Ce syndicat regroupe les agro-industries opérant au Cameroun. Cet organisme est créé en 1993 et compte actuellement quatre membres, à savoir la SOCAPALM-SPFS, la C.D.C, la SAFACAM, et la PAMOL. Il permet aux agro-industries de mettre en commun l'état de leur stock d'huile

21 Communication lors d'une réunion du Groupe de travail sur le développement durable du palmier à huile au Cameroun, à Yaoundé le 30 septembre 2013. 
afin d'anticiper les périodes de pénuries dans les stocks nationaux. Ceci a pour but de limiter les importations par les industriels de la seconde transformation qui redoutent les pénuries de matière première. Ce syndicat défend aussi les intérêts des agro-industries auprès de l'État.

\section{L'Association des Transformateurs des Produits Oléagineux (ATPO)}

Cette association est créée en 2000 et regroupe les transformateurs d'huile de palme du Cameroun. Ces membres sont essentiellement des savonneries et des raffineries industrielles. Cette association planifie ses besoins en matière première et les répercute au SNPHPC et à l'État pour être autorisée à importer de l'huile avec une taxation réduite ; elle défend aussi les intérêts de la profession auprès de l'État.

\section{L'Union des Exploitants de Palmier à Huile du Cameroun (UNEXPALM)}

Créée en décembre 2000, UNEXPALM est une association regroupant les planteurs nationaux de palmier à huile. En 2009, Elle compte 2000 membres provenant des sept régions du Sud Cameroun. Créée par un ancien ministre, l'UNEXPALM a un fonctionnement très administratif top-down qui convient parfaitement aux élites, mais ne correspond guère aux attentes des agriculteurs familiaux qui ne s'y sentent pas toujours représentés. Elle jouit cependant d'une notoriété qui ne cesse de s'affirmer au plan national et international.

\section{La Plateforme Nationale des Organisations de Producteurs Agro-sylvo-pastoraux du Cameroun (PLANOPAC).}

La PLANOPAC est une association interprofessionnelle créée en octobre 2007 regroupant les associations de producteurs ruraux (agriculteurs, sylviculteurs, éleveurs et pêcheurs). C'est une sorte de centrale syndicale dont l'objectif principal est la défense des intérêts des producteurs. Elle représente également ces derniers à travers leurs organisations professionnelles. Elle se compose de plateformes régionales et de sections par produit. Ainsi, il existe une section palmier au sein de la PLANOPAC qui complète l'action d'UNEXPALM.

\subsection{Le retour de l'intervention de l'État}

Dans le cadre de la mise en ouvre de la nouvelle politique agricole définie en 1990, l'État camerounais initie un certain nombre de projets en faveur du développement des palmeraies villageoises. L'État met également en place un cadre réglementaire visant à améliorer la compétitivité de la filière de l'huile de palme au Cameroun et à limiter les importations d'huile asiatique brute ou raffinée.

\subsubsection{De nombreux projets gouvernementaux mais de portée limitée}

\section{Le projet PEPIPALM}

Financé par l'Union européenne sur fonds STABEX et coordonné par l'ex-MINAGRI (Ministère de l'Agriculture), le projet PEPIPALM a pour objectif la constitution d'un réseau de pépiniéristes privés dans les départements de la Sanaga-Maritime et du Nyong-et-Kéllé. Il débute en 1997 et s'achève en 2002. Ses promoteurs ambitionnent de mettre à la disposition des planteurs, des plants de palmiers sélectionnés de qualité à hauteur de 1300 francs CFA l'unité. Ce projet permet de former 17 pépiniéristes privés qui reçoivent par ailleurs un accompagnement technique et financier. Entre 1997 et 2002, le projet produit 247000 plants (Bakoumé et al., 2002). Cependant, des retards dans la livraison des plants, le développement des pépinières concurrentes utilisant le matériel de la station de la Dibamba et la reprise des cours du cacao précipitent l'arrêt du projet en 2002. En plus, il semble que les prix pratiqués (1 300/le plant) ne sont pas à la portée des petits planteurs. Il en résulte que les principaux acheteurs de plants PEPIPALM sont les élites urbaines. Actuellement, un seul des 17 pépiniéristes poursuit cette activité.

\section{Le Projet Pôle de Développement Rural (PPDR)}

Le PPDR est un projet financé par l'Union européenne sur fond STABEX. Il est mis en œuvre sous la tutelle du Ministère en charge de l'agriculture entre 1992 et 2004 et couvre les pôles de Sa'a, Ntui dans la région du Centre et Sangmélima dans la région du Sud. Le principal objectif du PPDR est la valorisation du monde 


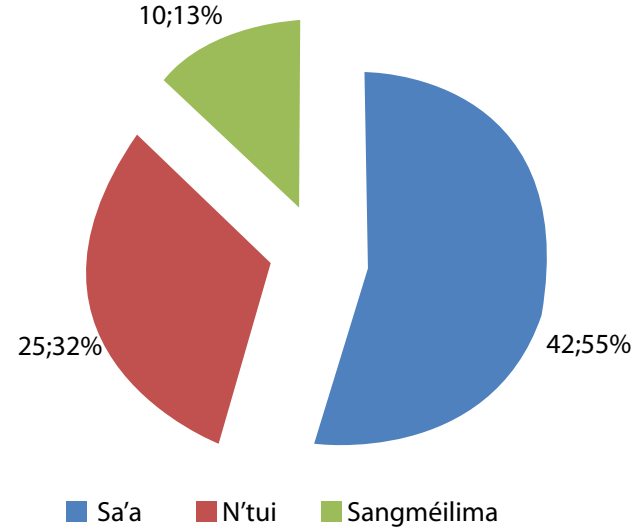

Figure 15 : Répartition entre les 3 sites des organisations paysannes de palmier à huile soutenues par le PPDR

Source : Agence centrale PPDR, 2000

rural afin de stimuler les capacités propres des populations rurales à se prendre en charge et à améliorer leurs conditions de vie (GOPA Consultants, 1999). De manière spécifique, il s'agit d'augmenter la production agricole et de maintenir l'autosuffisance alimentaire, d'améliorer les conditions de vie en milieu rural et de contribuer à la réduction de l'exode rural et de dynamiser l'organisation des communautés villageoises dans les sites du projet. Le PPDR couvre plusieurs spéculations agricoles. La mise en œuvre du projet est assurée par des interfaces qui sont au nombre de trois, dont une par pôle. Le Centre de Développement Auto-Centré (CEDAC) est choisi pour servir d'intermédiaire sur le site de Sangmélima, le Centre Rural d'appui Technique (CRAT) sur celui de Sa'a et la Congrégation des Frères des Écoles chrétiennes (CFEC) se voit confier le site de N'tui. Toutes ces structures sont d'obédience chrétienne favorable au développement du palmier à huile qui fait partie des volets sélectionnés par le projet. Dans le domaine du palmier à huile, les activités principales du PPDR tournent autour de la création des pépinières villageoises, l'organisation du mouvement paysan en vue des achats et des ventes groupés de semences sélectionnées et les subventions à l'achat des presses artisanales motorisées. Ici également, les élites sont les principaux acheteurs des plants sélectionnés. L'appui du PPDR est essentiellement accordé aux organisations paysannes. Entre 1992 et 1999, le PPDR a appuyé 77 organisations paysannes dans les trois pôles d'activités (figure 17).
Le programme de Développement des Palmeraies villageoises (PDPV)

Logé au MINADER, le PDPV est mis en

place en 2004 avec pour objectif principal l'amélioration du niveau de vie des paysans par une augmentation des revenus stables tirés de la production de l'huile de palme. Il a pour maître d'ouvrage le MINADER tandis que le maitre d'œuvre est l'Union des Exploitants de Palmier à Huile du Cameroun (UNEXPALM). Pendant la première phase d'exécution, ce programme permet la distribution des plants de palmiers à huile sélectionnés à 7600 paysans à raison d'un hectare par planteur ainsi que la formation de 700 producteurs et 500 encadreurs locaux du MINADER sur l'implantation et la conduite technique d'une plantation de palmiers à huile et sur la gestion financière d'une palmeraie. Au début de la première phase du projet, l'offre en plants sélectionnés était inférieure aux besoins du projet si bien que des achats de plants ont été effectués là où des plants étaient disponibles. Au cours de la deuxième phase qui a commencé en 2012, le PDPV vise à accroître la production nationale d'huile de palme. De manière spécifique, il s'agit :

- d'améliorer la structuration et l'organisation de la palmeraie villageoise ;

- d'étendre les superficies cultivées dans les bassins de production et d'en améliorer l'exploitation ;

- de créer des unités industrielles de transformation des régimes ;

- d'entretenir et de créer des pistes de collecte afin de permettre l'approvisionnement des unités de transformation des régimes de palmes ;

- de faciliter l'accès au crédit de plantation, de campagne pour l'achat des engrais.

Le PDPV est actuellement engagé dans un processus de recensement des plantations villageoises sur toute l'étendue du territoire national avec un accent particulier sur les régions du Littoral, du Sud-Ouest, du Sud, du Centre, de l'Est et de l'Ouest. Ce recensement des superficies et du nombre de petits planteurs s'effectue par enquête auprès des planteurs. Pour le faire, le PDPV a recours aux prestataires de services au terme d'un processus de passation de marché. Dans les arrondissements d'EDEA I, EDEA II et DIBAMBA par exemple, cette tâche a été confiée à la structure dénommée PROMOTEURS DU DEVELOPPEMENT RURAL (PRODERU) 
Ce recensement des superficies et du nombre de petits planteurs s'effectue par enquête. Le type de matériel végétal planté est évalué auprès des agriculteurs en leur demandant comment ils se sont fournis en plants ou en graines prégermées.

\section{Programme d'Amélioration de la Compétitivité Agricole (PACA)}

Le programme PACA est sous la tutelle du MINADER. Il est financé par la Banque mondiale et intéresse essentiellement les petits producteurs et les organisations de producteurs. Il vise six filières prioritaires à savoir le riz, le maïs, la banane plantain, le porc, la volaille et le palmier à huile. Concernant ces productions agricoles, le projet intervient dans sept des dix Régions du Cameroun, en l'occurrence le Centre, le Sud, l'Extrême Nord, le Nord, l'Est, l'Ouest, et le Littoral. En fonction de leur potentiel pour les filières ci-dessus mentionnées, 30 départements sont sélectionnés pour la mise en œuvre du projet. Le volet palmier n'a pas encore démarré, mais le projet entend créer plus de 4000 ha de palmeraies villageoises permettant d'accroître la production de régimes à $56000 \mathrm{t}$ sur la base d'un rendement de 14 tonnes par ha, ce qui équivaut à 11760 tonnes huile de palme brute (PACA, 2009). Ce projet est particulièrement ambitieux, car actuellement seules les agro-industries privées parviennent à obtenir de tels rendements moyens sur 4000 ha.

\section{Le Projet d'Amélioration de la Productivité et de la Compétitivité de la filière Palmier à Huile en Afrique centrale et de l'ouest (APROCOM-Ph)}

Ce projet est sous la coordination du ministère des Mines, de l'Industrie et du Développement Technologique (MINMIDT) en partenariat avec le Fonds des Nations Unies pour le Développement Industriel (ONUDI) et d'autres institutions publiques (MINEFI, MINADER, MINEPAT), MINCOMMERCE). Il bénéficie d'une enveloppe budgétaire de 2,5 milliards de francs CFA. Ce projet vise à résorber la pénurie d'huile de palme au Cameroun et dans la sous-région CEMAC. C'est ainsi qu'en mai 2012, le Secrétaire Général du ministère des Mines, de l'Industrie et du Développement Technologique (MINMIDT) a procédé à la pose de la première pierre d'une huilerie d'une capacité de traitement de $2 \mathrm{t}$ de régimes/h à Sombo dans l'arrondissement de Dibang, département du Nyong-et-Kéllé, région du Centre. Le coût de l'ouvrage est estimé à plus de 500 millions Francs CFA. D'autres localités devaient également bénéficier d'une huilerie moderne à l'instar de Green Valley plantation dans le département du Fako au Sud-Ouest, Mkpot dans le département de la Manyu toujours au SudOuest et Ngie dans le Nord-Ouest (http://www. news.mboa.info/economie/fr/).

\section{Le programme semencier national}

Ce programme est sous la tutelle du MINRESI et couvre plusieurs spéculations agricoles. Pour ce qui concerne le palmier à huile, il se décline en un sous-programme appelé le Programme National de Production des Semences de Palmier à Huile Sélectionnées (PNPSPHS) mis en œuvre par le Centre Spécialisé de Recherche sur Palmier à Huile (CEREPAH) de la Dibamba. Au sein de l'IRAD, il existe un Comité de recherche-développement qui est un organe consultatif du Comité de pilotage du PNPSPHS chargé de la production de semences de palmier à huile. Lancé le 17 décembre 2009 à Dizanguè par le ministre de la Recherche scientifique et de l'Innovation, le PNPSPHS a pour objectif de faciliter à l'horizon 2014 la plantation de près de 75000 ha de palmiers à huile à hauts rendements grâce à l'augmentation en quantité et en qualité de la production de semences sélectionnées et l'appui technique aux acteurs de la filière. Il s'agit de produire en 5 ans, 22 millions de graines germées à partir du patrimoine génétique existant au CEREPAH de la Dibamba, de fournir du matériel végétal et des technologies appropriées et un appui technique aux différents acteurs de la filière palmier à huile et de mettre en place un système de gestion collégiale et par objectif du Programme. Le coût global de ce Programme qui doit durer cinq (05) ans s'élève à près de 4 milliards de FCFA et les principaux partenaires sont entre autres : le ministère de la Recherche Scientifique et de l'Innovation, le ministère des Finances, la SOCAPALM, la CDC, PAMOL, SPFS, SAFACAM, les Organisations paysannes et autres Groupements de petits planteurs (www.iradcameroon.org).

\subsubsection{La mise en place d'un cadre réglementaire incitatif et protectionniste}

En dehors de ces projets, l'État définit un cadre juridique et réglementaire pour organiser et protéger le marché local de l'huile de palme. Rappelons d'abord que le développement de l'agriculture et de l'élæiculture ainsi que la 
promotion de l'agro-industrie figurent parmi les secteurs bénéficiant des incitations spécifiques ${ }^{22}$. En plus, l'huile de palme figure parmi les produits dont les prix et tarifs sont soumis à la procédure d'homologation préalable ${ }^{23}$. C'est ainsi qu'un comité ad hoc de régulation de la filière huile de palme est institué en $2004^{24}$ pour fixer le prix de l'huile palme en fonction de la conjoncture. Il est constitué des producteurs, des transformateurs et des pouvoirs publics. Un prix maximal de cession de l'huile de palme brute par les industriels aux grossistes est ainsi fixé par l'État et varie en fonction des années et des contextes. Cette mesure permet de déconnecter le prix de l'huile de palme du cours international (souvent plus bas que le cours intérieur) dans l'optique de dynamiser la production intérieure. Ce prix maximal sert également de référence au calcul du prix d'achat des régimes aux petits planteurs par les huileries (PACA, 2009).

L'industrie de raffinage est également protégée à travers la décision $\mathrm{N}^{\circ} 488 \mathrm{MINFI} / \mathrm{DGD} \mathrm{du}$ 27 mars 2009 fixant le prix de référence pour la taxation des huiles importées au Cameroun. Ce prix est fixé à $1500 \mathrm{~F}$ CFA le litre. L'importation de l'huile de palme brute est aussi protégée par une taxe à l'importation de $30 \%$ avec possibilité de réduction à $10 \%$ sur avis du comité ad hoc (PACA, 2009) et après un constat de pénurie attestée.

\subsection{De nombreuses initiatives privées : le projet de villagisation de la SOCAPALM}

Les acteurs privés participent également à la dynamisation de la filière palmier à huile à travers la mise en ouvre de nombreux projets. C'est le cas de la SOCAPALM qui initie le "projet de villagisation " sur son site d'Eséka au lendemain de sa privatisation. En effet, jusqu'en 2000, la SOCAPALM est une société

22 Loi n ${ }^{\circ} 2013 / 004$ du 18 avril 2013 fixant les incitations à l'investissement privé en République du Cameroun.

23 Arrêté $\mathrm{N}^{\circ} 016$ a/MINDIC/DPMPC/SDEL/SLR du 24 juin 2004 portant fixation de la liste des produits et services dont les prix et tarifs sont soumis à la procédure d'homologation préalable.

24 Arrêté $\mathrm{N}^{\circ}$ 12/MINDIC/CAD du 21 mars 2004 portant création et organisation du comité ad hoc en charge de la régularisation de la filière huile de Palme au Cameroun.
d'État créée en 1968. Frappée par la crise des années 1980, elle est privatisée le 30 juin 2000. L'État camerounais cède une grande partie de ses actions à PALMERAIES DU CAMEROUN (PALMCAM) détenue elle-même à 63,72\% par le Groupe INTERCULTURES / SOCFINAL (groupe Fabri / Bolloré) et à 36,28 \% par la Société Financière et Commerciale «SFC» (Groupe Monthe). Les $3 \%$ des actions de SOCAPALM restantes sont portées par PALMCAM au profit du personnel de SOCAPALM (SOCAPALM, 2009). Cette cession sanctionne la liquidation totale de la société (Mbouhnoum, 2009). En 2004, la SOCAPALM signe avec l'État du Cameroun un bail emphytéotique d'une durée de 60 ans en vue de l'exploitation de 58063 hectares de terres rurales. Sur son site d'Eséka, la société met en fermage 2600 hectares de vieilles palmeraies au profit des planteurs riverains pour une période de 25 ans. Les acquéreurs bénéficient de l'encadrement technique de la SOCAPALM qui leur fournit par ailleurs les engrais, les plants et quelques produits phytosanitaires à la charge des planteurs. En retour, les acquéreurs organisés en coopérative sont obligés de livrer leurs régimes à l'huilerie d'Eséka. En outre, ces derniers doivent procéder à la replantation à leurs frais des lots acquis. Le remboursement des crédits alloués aux planteurs s'étale sur 8 ans et s'effectue par l'intermédiaire de la MITFUND, un organisme d'AFRILAND FIRST BANK installé sur le site de la SOCAPALM à Eséka. Le projet démarre véritablement en 2007/2008 et compte 102 adhérents ayant acquis des lots d'une superficie moyenne de 8 ha et pouvant atteindre 300 ha dans certains cas. Plus de $90 \%$ des acquéreurs sont des élites urbaines (Mbouhnoum, 2009). Ce projet est une initiative pilote qui est censée s'étendre sur tous les sites SOCAPALM, mais reste limitée à ce site pour le moment.

\subsection{Les demandes de nouvelles concessions élæicoles}

Au cours des deux dernières décennies, le contexte mondial du palmier à huile est marqué par une augmentation significative de la demande en huile de palme. Cependant, chez les grands producteurs asiatiques que sont la Malaisie et l'Indonésie, les terres se font de plus en plus rares et surtout plus difficilement accessibles en raison de la pression des ONG environnementales ce qui complique 
la conversion des terres forestières. En plus, la problématique de la REDD incite les grandes compagnies asiatiques à diversifier leurs zones de production. L'intérêt des compagnies pour le Cameroun s'inscrit donc dans une logique de contournement des pressions environnementales dans leurs pays d'origine. Les pays du golfe de Guinée en général et le Cameroun en particulier, compte tenu de leurs potentialités naturelles et humaines, constituent une des cibles géographiques de ces nouveaux investisseurs étrangers. Jusqu'en 2012, au moins 6 sociétés ont déposé des demandes de concessions auprès des autorités camerounaises. Ces demandes d'acquisitions foncières sont de l'ordre d'1 million d'hectares dans la zone forestière du Sud Cameroun (Hoyle et Levang, 2012). Il est toutefois difficile de faire la part des effets d'annonces et des exagérations journalistiques en raison d'un total manque de transparence des principaux acteurs concernés par ces transactions.

\subsection{La restructuration et la quête de la durabilité de la filière palmier à huile}

En définitive, les années 1990 constituent une période amère pour l'économie camerounaise. Elles correspondent à la mise en œuvre des programmes d'ajustements structurels au Cameroun dont l'un des corollaires est le désengagement de l'État des secteurs productifs, ce qui entraîne la privatisation des entreprises publiques et parapubliques et l'arrêt des subventions agricoles de l'État. Cette situation est à l'origine de profondes mutations dans la filière palmier à huile et de sa restructuration. Au niveau des producteurs, on assiste à une diversification des acteurs impliqués dans la filière avec une présence marquée des producteurs indépendants et plus particulièrement des élites urbaines mais aussi rurales. Du côté des transformateurs, une compétition rude s'installe d'une part entre les transformations industrielles et artisanales et d'autre part entre les anciennes industries de seconde transformation (CCC, SRC-MAYA) et les nouvelles (AZUR, NOSA). Sur le plan spatial, l'avènement des presses artisanales permet le développement de palmeraies villageoises en dehors des bassins de production élæicoles traditionnels situés près des agro-industries. C'est à la fois une opportunité pour augmenter la production nationale, mais aussi pour le développement local en zone rurale. Les années 1990 constituent également le début de la restructuration de la filière palmier à huile avec la création des premières organisations professionnelles et interprofessionnelles. Malgré la crise et le désengagement de l'État, le gouvernement camerounais initie de nombreux projets à court et à moyen terme en même temps qu'il définit un cadre réglementaire visant à protéger le marché national de l'huile de palme et à redynamiser la filière palmier à huile. Dès les années 2000 enfin, la durabilité des pratiques élæicoles préoccupe les autorités camerounaises qui initient la définition d'une stratégie de développement durable de la filière palmier à huile dans un contexte marqué par la course à l'émergence et de nombreuses demandes de concessions élæicoles par des multinationales étrangères et principalement asiatiques et américaines. La perspective de la mise en œuvre de cette stratégie ouvre la possibilité aux acteurs de discuter des problèmes qui frappent le secteur et de trouver des solutions concrètes et réalisables. Sur cette lancée, un accent particulier doit être mis sur les palmeraies villageoises qui contribuent directement à l'amélioration des conditions de vies des masses rurales. 


\section{Conclusion générale : Enjeux et perspectives du secteur élæicole camerounais}

L'exploitation du palmier à huile commence par une simple cueillette traditionnelle opérée par les communautés de la zone forestière du Cameroun. Avec l'arrivée des Européens à la fin du XIX siècle, la culture du palmier à huile en plantation débute et connaît un fort développement au lendemain de la Deuxième Guerre mondiale avec la création de la CDC et de la SOCAPALM. La modernisation de la filière palmier à huile initiée au cours de la même période est interrompue à la fin des années 1980 à cause de la crise économique à l'origine du désengagement de l'État. Cette situation place la filière palmier à huile au centre de profondes mutations qui perdurent de nos jours et qui contribuent à la restructuration et à l'amélioration de la compétitivité de la filière. Le rôle de l'État dans cette dynamique est déterminant. En se désengageant de la production et de la commercialisation des produits du palmier, l'État devient un propulseur, un facilitateur et un régulateur de l'activité élæicole à travers la mise en œuvre de nombreux projets et la définition d'un cadre institutionnel et légal favorable au développement de l'exploitation du palmier à huile.

Le principal enjeu de développement durable du secteur palmier à huile au Cameroun reste la satisfaction de la demande intérieure en huile de palme qui ne cesse d'augmenter avec la croissance démographique et industrielle. La dynamique de croissance actuelle mérite ainsi d'être maintenue. Ceci passe entre autres par un meilleur encadrement des petits planteurs et la mise à la disposition de ces derniers d'un matériel végétal performant qui leur permettent d'atteindre des rendements de l'ordre de ceux obtenus par les agroindustries. L'exploitation optimale des plantations existantes constitue également un gain potentiel de production particulièrement important dans les agro-industries publiques, mais surtout dans les plantations villageoises caractérisées par des performances très hétérogènes.

Le partenariat gagnant-gagnant entre les producteurs villageois et les agro-industries constitue également un défi important pour l'augmentation de la production d'huile de palme au Cameroun. C'est une réelle opportunité de diffusion des innovations en direction des planteurs villageois, notamment en ce qui concerne l'utilisation d'un matériel végétal performant et des engrais appropriés qui sont les 2 clefs de la performance agronomique, sans oublier l'extraction d'huile de palme brute par les huileries, performante sur le plan technologique et environnemental. Ce partenariat gagnant-gagnant est également une belle opportunité de crédit de campagne grâce à des facilités de paiement des engrais qui fidélisent les petits planteurs. Il en résulte forcément une amélioration de l'offre villageoise aussi bien en régimes qu'en huile de palme brute et de palmiste sans forcément nécessiter l'augmentation des surfaces cultivées. Il apparaît dès lors comme un moyen de lutte contre la déforestation. Le Cameroun a déjà expérimenté ce type d'agriculture contractuelle dans les années 1970 et 1980 . Tout en protégeant l'indépendance des petits planteurs, il est possible de reproduire ce modèle en l'adaptant au contexte actuel. C'est d'ailleurs ce que font les agroindustries privées dans une démarche informelle qui se limite à des crédits de campagne pour limiter les risques, et s'étend parfois à la fourniture de plants ou de semences sélectionnées, mais surtout pour les élites. La contractualisation ouvrirait de nouvelles opportunités à ce partenariat.

Toutefois, les problèmes de tenure restent au centre du développement élæicole au Cameroun. Les modes d'acquisition foncière et les logiques des acteurs en présence constituent un réel motif de 
préoccupation, car ils sont à l'origine de conflits divers, aussi bien entre les différents modes d'occupation du sol (élæiculture/conservation/ exploitation forestière/agriculture vivrière) qu'entre les parties prenantes du développement élæicole du Cameroun (élites, familles, communautés villageoises, agro-industries/État). La perte des droits fonciers se traduit également par une perte de revenus pour les communautés villageoises par l'arrêt de l'exploitation du bois ou des produits forestiers non ligneux. Une expansion incontrôlée de l'élæiculture est susceptible d'occasionner une pénurie de terre aggravée par l'urbanisation galopante. Celle-ci risque de rattraper les zones d'extension du palmier à huile et d'encourager le vol des régimes dans les plantations. La révision et l'harmonisation des textes légaux, la promotion de la participation et de la transparence dans les transactions foncières et une meilleure coordination des acteurs sont nécessaires pour faire face à ces enjeux.

Par ailleurs, la promotion de l'emploi est également préoccupante. L'élæiculture doit générer des emplois aussi bien dans le secteur agro-industriel que dans le secteur villageois ainsi que dans les domaines de la commercialisation et de la fourniture de divers équipements (grillage, plants pour les pépiniéristes, outillage agricole, sachets, etc.). Cette problématique intéresse particulièrement les jeunes, compte tenu des difficultés d'accès au foncier, de l'investissement économique et humain que requièrent l'implantation puis la conduite juvénile d'une palmeraie, du manque de capitaux nécessaires et de l'impossibilité d'accès au crédit de plantation. Dans la perspective de création d'emplois décents dans la filière palmier à huile, la formation des acteurs revêt une importance capitale aussi bien en aval qu'en amont de la filière palmier à huile.

Pour ce faire, le développement du palmier à huile doit être résolument tourné vers la durabilité. C'est pourquoi, dans le cadre de la politique de l'émergence à l'horizon 2035, les pouvoirs publics ont initié la définition d'une stratégie nationale de développement durable du palmier à huile et entendent s'engager dans le processus de certification RSPO. En effet, la culture du palmier à huile occupe une place non négligeable dans la politique d'émergence du Cameroun à l'horizon 2035 telle que déclinée dans le Document de Stratégie pour la Croissance et l'Emploi (DSCE) publié en 2009. Le gouvernement entend doubler la production d'huile de palme de 2005 (elle est alors évaluée à 177000 t) à l'horizon 2015 (MINADER, 2005). Avec un taux de croissance de l'ordre de $10 \%$, le gouvernement ambitionne d'augmenter les superficies des palmeraies sélectionnées à 203000 ha et la production d'huile de palme à $450000 \mathrm{t}$ en 2020 (MINADER, 2005). Cependant, tenant compte des enjeux socioéconomiques et environnementaux de la filière palmier à huile, son développement durable préoccupe les acteurs à la fois publics et privés qui prennent conscience de la nécessité de définir une stratégie nationale de développement durable du palmier à huile et de s'engager dans la certification.

Depuis 2012, plusieurs rencontres regroupant les divers acteurs impliqués dans le développement du palmier à huile (MINADER, organisations professionnelles, ONG, et chercheurs) ont été organisées à l'initiative du WWF-CARPO et du MINADER pour réfléchir sur la nécessité de promouvoir le développement durable du palmier à huile au Cameroun. Un groupe de travail a été nommé par le MINADER en 2013 pour préparer cette stratégie ${ }^{25}$. Ce groupe intègre des représentants de différents ministères (MINAGRI, MINFOF, MINDAF, etc.), des organisations professionnelles liées à la filière (UNEXPALM, ATPO), des ONG (WWF, PROFOREST, CED), des centres de recherche (CIRAD, CIFOR, IRD) et des représentants du secteur académique (Université de Yaoundé 1, Université de Douala).

La stratégie de développement durable du palmier à huile rentre dans une dynamique globale de réformes institutionnelles au Cameroun. Une loi d'orientation d'aménagement du territoire est ainsi promulguée en 2011 afin d'harmoniser l'occupation et la mise en valeur de l'espace. Des réformes foncière et forestière sont également en cours depuis 2012 de même que la création d'une

25 Décision $\mathrm{N}^{\circ}$ 00250/CAB/ MINADER du 29 juillet 2013 portant création au sein du ministère de l'Agriculture et du Développement Rural d'un comité chargé de l'élaboration de la stratégie nationale de développement durable de la filière palmier à huile au Cameroun. 
Agence d'Aménagement et de Gestion des Terres Agricoles (AGTA) au sein du MINADER ${ }^{26}$.

Les enjeux de développement à grande échelle d'une filière interpellent les acteurs et les scientifiques sur les risques et opportunités en termes d'impacts socioéconomiques et environnementaux. Dans ce contexte, le projet de recherche SPOP (Sustainable Palm Oil Production project) financé par l'Agence Nationale de la Recherche française (ANR) et coordonné par le CIRAD, en partenariat avec le CIFOR et l'IRD vise à caractériser les systèmes de production élæicoles indonésiens et camerounais afin de comparer leurs performances réciproques tant sur le plan agronomique que socioenvironnemental. L'approche combine une analyse fine des impacts en 3D c'est-à-dire tridimensionnels (économiques, sociaux et environnementaux) différenciés des différents systèmes de culture du palmier à huile à l'échelle de la parcelle et une analyse des dynamiques d'utilisation des sols à l'échelle territoriale.

Pour promouvoir la production de l'huile de palme durable, la stratégie nationale de développement durable du palmier à huile peut s'appuyer sur l'interprétation et l'application des principes et des critères de la Roundtable for Sustainable Palm Oil (RSPO). La RSPO est une initiative mise en place en 2004 dont l'objectif est de promouvoir la production et l'utilisation durable des produits du palmier à huile par l'application de références globales crédibles et l'application de principes et critères de durabilité. Elle réunit les parties prenantes des secteurs impliqués dans le développement du palmier à huile. L'huile de palme est qualifiée de durable lorsqu' elle est certifiée conforme aux principes et aux critères RSPO (voir www.rspo.org/page/513), entre autres respectant les conditions d'existence des communautés rurales, la contribution au développement local, régional et national et la protection des zones à haute valeur de conservation ${ }^{27}$. Pour entrer dans le processus RSPO, les États et les acteurs sont appelés à procéder à des interprétations nationales des principes et critères RSPO. Au Cameroun, nous sommes encore pour le moment dans la phase de sensibilisation des acteurs et parties prenantes de la filière à la certification RSPO.

Cependant, la mise en place d'une concertation entre les différents acteurs de la filière palmier s'avère nécessaire en même temps que la poursuite de l'organisation des producteurs. Les organisations professionnelles actuelles et futures doivent jouer leur rôle de clé de voûte du développement rural à travers la formation, l'information des acteurs et la défense des intérêts de tous les types d'agricultures très diversifiés qui se regroupent derrière l'appellation "petits planteurs». Le dynamisme des organisations paysannes est indispensable dans la collecte des statistiques des petits planteurs indépendants qui restent inconnues à ce jour, la formation et l'information des acteurs, de même que l'identification des thèmes de recherche pertinents et l'organisation de la collecte des récoltes et la définition des partenariats.

En revanche, l'histoire a montré que les agroindustries privées sont souvent plus efficaces et plus sûres que les Services publics pour la diffusion de véritables plants sélectionnés bien développés et livrés à la bonne période, la fourniture d'engrais à des prix attractifs avec des facilités de paiement pour les petits planteurs fidélisés. Seuls les crédits avec caution solidaires, une gestion locale, transparente et de proximité peuvent espérer fonctionner au Cameroun.
26 Communication du représentant du ministre de l'Agriculture et du Développement Rural lors d'un séminaire sur le développement durable du palmier à huile au Cameroun organisé à Yaoundé les 11 et 12 décembre 2012 par le WWF/MINADER.
27 Les zones de haute valeur de conservation se réferent aux valeurs biologiques, écologiques, sociales et culturelles considérées comme significatives à l'échelle locale, régionale et nationale ou d'importance critique (RSPO, 2012). Visiter le site www.hcvnetwork.org pour de plus amples informations. 


\section{Bibliographie}

Adams, J., 1942 Le palmier à huile, Paris, Société d'édition géographique maritime coloniale.

Agence centrale PPDR 2004 Capitalisation des activités du PPDR. Rapport final, Yaoundé.

Anonyme 1980. Anthologie des discours de 1957 à 1979, S.E. Ahmadou Ahidjo, Paris, Les nouvelles éditions africaines.

Awoumou Amougou Jean de Dieu 2006 La Libéralisation des marchés et le développement durable en Afrique : le cas du secteur agricole au Cameroun; Mémoire de Master en Administration Publique, Université Louis Pasteur Strasbourg, Faculté des Sciences économiques et de Gestion, 63 p.

Ayuketang, D.E., 2010 Plantation agriculture in Fako division and Bakweri land problem 1884-2006 : an historical survey, Dissertation of a master degree in History, Université de Yaoundé I, 158 p.

Bakoume, C., Jannot, C., Rafflegeau, S., Ndigui, B., Weise, S. 2002 Études complémentaires sur la relance des filières hévéa et palmier à huile, rapport, $80 \mathrm{p}$.

Banque Européenne d'Investissement 1964 La République Fédérale au Cameroun : Situation économique en 1963, $201 \mathrm{p}$.

Banque mondiale 1977 Cameroon Appraisal of a Second CAMDEV Project.

Banque mondiale 1977 Évaluation du deuxième projet SOCAPALM.

Bidjeck, M.P. 2002 La culture du palmier à huile et le rôle de la SOCAPALM dans le développement de la région d'Eséka : 1960-1991, $112 \mathrm{p}$.

Bidjeck, M.P. 2007 La dynamique des cultures de rente dans la région d'Eséka de la période coloniale à l'an 2000.Mémoire de DEA en Histoire, Université de Yaoundé I, 99 p.

BIRD/IDA 1967 Projet de la Cameroons Development Corporation, République Fédérale du Cameroun. Département des projets, $48 \mathrm{p}$.

Botele Ndiomo, F. 1973 L'incidence du palmier à huile sur le développement économique et social du Cameroun. Mémoire de Licence en sciences économiques, Université de Yaoundé. Bouchaud, J. 1952 La côte du Cameroun dans l'histoire et la cartographie des origines à l'annexion allemande. Mémoires de l'institut français d'Afrique Noire, Centre Cameroun, $\mathrm{N}^{\circ} 5,212 \mathrm{p}$.

Busia, K.A. 1956 La situation et les aspirations actuelles des élites de la Côte de l'Or, Bulletin international des sciences sociales, Vol. 8 (3).

Capot-Rey, P. 1968 Premier programme de développement de la culture du palmier à huile au Cameroun oriental, secteur d'Eséka, étude d'environnement humain, Paris.

Capot-Rey, P., Audebert, D., Carle, H. et de Rostolan, L. 1968 Étude agrosocioéconomique dans le secteur d'Eséka, Ministère du Plan et du Développement, 37 p. mult., 13 tabl.

CDC 1987 Annual report.

Champaud, J. 1966 L'économie cacaoyère du Cameroun. Cah. O.R.S.T.O.M., sér. Sci. hum., III, 3-1966, 20 p.

Champaud, J. 1972 Genèse et typologie des villes du Cameroun de l'ouest. Communication Symposium Union Géographique Internationale sur les villes dans les pays en voie de développement, août 1972. O.R.S.T.O.M., sér. SC. hum., IX, 3, p. 325-336.

Courade, G. 1979 Victoria Bota : Croissance urbaine et immigration, Paris, Travaux de l'ORSTOM, $124 \mathrm{p}$.

Courade, G. 1984 Des complexes qui coûtent cher : la priorité agro-industrielle dans l'agriculture camerounaise, Politique africaine 14, p. 75-91

Ekobena Iya, F. E. 2012 Exploitation élæicole au Cameroun : épuisement d'un Modèle de développement inefficace et ses conséquences socio-économiques, Mémoire maîtrise en science politique, Université du Québec à Montréal, Canada. 158 p. 
Elong, J. G. 2003 Les plantations villageoises de palmier à huile de la SOCAPALM dans le basMoungo (Cameroun) : un projet mal intégré aux préoccupations des paysans. Les Cahiers d'Outre-Mer : 224 (Octobre-Décembre 2003). http://com.revues.org/index738.html

Elong, J. G. 2011 L'élite urbaine dans le paysage agricole africain : exemples camerounais et sénégalais, Yaoundé, Harmattan Paris. 180 p.

Esua Y.W. Fossung, 2001 The role of plantation agriculture in regional development: the case of the Cameroon development corporation. Dans Readings in Geography, p. 370-388.

Etoga Eily 1971 Sur les chemins du développement : essai des faits économiques du Cameroun, Yaoundé, CEPMAE.

Feintrenie, L. 2012 Transfer of the Asian model of oil palm development: from Indonesia to Cameroon. World Bank conference on land and poverty, April 23-25, Washington DC. 19 p.

Fèvre, E., 2002 .Etude sur la relance des filières hévéa et palmier à huile : Analyse de la viabilité socioéconomique des systèmes d'exploitation de l'hévéa et du palmier à huile. Rapport provisoire, Revue du secteur rural, FAO/CP, 55 p.

Fouda Moulende, T., 2003. Les mécanismes de financement en milieu rural camerounais. Une analyse des déterminants de la demande de services financiers des ménages. Thèse de doctorat en sciences économiques, Université de Versailles Saint-Quentin-en-Yvelines, France. 379 p.

Franqueville, A. 1952 Atlas régional Sud-Ouest 1 : échelles $1 / 500000$ et $1 / 1000000$ : République unie du Cameroun, ORSTOM, Centre de Yaoundé, 109 p.

Gardinier 1967 The British in the Cameroons 1919-1939, New Heaven, Yale University Press.

GOPA Consultants 1999 Rapport de faisabilité, de reformulation et d'orientation sur l'extension du projet " pôles de développement rural " version finale, Yaoundé. $15 \mathrm{p}$.

Hartley, C.W.S. 1988 The oil palm (Elais guineensis Jacq.). Tropical Agriculture Series. ESSEX, Longman Scientific and Technical ed. Document.

Hirsch, R.D. 1999 La filière palmier à huile au Cameroun dans une perspective de relance. Rapport d'étude, Agence Française de développement, $77 \mathrm{p}$.
Hoyle, D., Levang, P. 2012 Le développement du palmier à huile au Cameroun.

IRAD 2008 Deuxième rapport sur la situation des ressources phylogénétiques pour l'alimentation et l'agriculture au Cameroun, $93 \mathrm{p}$.

Institut National de Cartographie 2011 Atlas national de développement physique du Cameroun.

Konings, P. 1979 L'État, l'agro-industrie et la paysannerie au Cameroun. Traduit de l'anglais par R. Buijtenhuijs, Agro-industrie, p. 120-137.

Koenings, P. 1993 Contract farming and capital accumulation in Cameroon: The case of the CDC smallholders schemes. p. 217-239.

Koenings, P. 1996 Privatisation of Agro-Industrial Parastatals and Anglophone Opposition dans Cameroon Journal of Commonwealth \& Comparative Politics, Vol. 34, N ${ }^{\circ} 3$, p. 199217, PUBLISHED BY FRANK CASS, LONDON.

Koenings, P. 1997 Agro-industry and regionalism in the South West Province of Cameroon during the national and political crisis, p. 289-305.

Kouo, F. 1979 Les répercussions de la crise économique de 1929 au Cameroun. In Afrika Zamani $\mathrm{n}^{\circ} 10$ et 11,1979 , p. 100-13.

Le Vine, V. T. 1964 The Cameroons from the mandate to independence. Berkeley et Los Angeles, University of California Press.

Lebailly, P., Tentchou, J. 2009 Etude sur la filière porteuse " Palmier à huile ».Rapport final, $96 \mathrm{p}$.

Levang, P. 2012 Elites et palmier, présentation pour l'IRD, Février 2012.

Limoigne, N. 2010 Mémoire des hommes, Mémoire des sols. Étude ethno-pédologique des usages paysans du Mont-Cameroun. Thèse de doctorat en Géographie, Université de Bordeaux, France, 431 p.

Madi, V. 2008 Les Défis environnementaux de la relance de la palmeraie villageoiese au Cameroun : Cas de la Sanaga maritime et de l'Océan. Mémoire de fin d'études de l'obtention du Diplôme d'Études Supérieures Spécialisées (DESS), Centre Régional d'Enseignement Spécialisé en Agriculture Forêt-Bois, Faculté d'agronomie et des Sciences Agricoles, Université de Dschang, Cameroun, $64 \mathrm{p}$.

Maley, J. 1999 L'expansion du palmier à huile (Elaéis guineensis) en Afrique centrale au 
cours des trois derniers millénaires : nouvelles données et interprétations. Dans Bahuchet, S., Bley, D., Pagezy, H., Vernezza Licht, N., l'Homme et la forêt tropicale, Travaux de la société d'écologie humaine, CNRS-Université de la Méditerranée, Marseille, France, p. 237-254.

Mbouhnoum, P. A. E. 2009 Étude comparative de deux formes de gestion participative d'une agro-industrie : la gestion étatique et privée de la SOCAPALM d'Eséka. Mémoire de DEA en Géographie, Université de Yaoundé I, 153 p.

Meunier, J, Gasgon, J.P. 1972 Le schéma général d'amélioration du palmier à huile à l'IRHO. Oléagineux, $27: 1-12$.

MINADER 2002 Revue du secteur rural, Yaoundé, Cameroun.

MINADER Agristat $\mathrm{n}^{\circ} 15$, rapport 2010.

MINADER 2011 Document de faisabilité de la deuxième phase du Programme de Développement des Plantations Villageoises, version revue et corrigée, Yaoundé.

MINADER 2012 Rapport d'étude d'identification et de caractérisation des bassins de production de palmier à huile dans le département de la Sanaga-Maritime. Programme de Développement des Plantations Villageoises, Yaoundé.

MINPLAPDAT 2005 Document de stratégie de Développement du secteur rural, $188 \mathrm{p}$.

Molua, H. N. 1985 The bakweri land problem 1884-1961: a case study, Thesis for the degree of Master of Arts, Université d'Ibadan, Nigéria, $171 \mathrm{p}$.

Mveng, E. 1985 Histoire du Cameroun, Paris, Présence africaine.

Ngoh, J. 1996 History of Cameroon since 1800, Limbé pressbook.

Ngom, E. 2011 Oil palm in Cameroon. Communication lors de l'échange Sud-Sud "Sharing what works in sustainable and equitable oil palm development ", organisé par le CIFOR à Bogor, Indonésie, 2127 septembre 2011

Ngom, E. 2013. Vers une stratégie nationale de développement durable de la filière palmier a huile au Cameroun, MINADER, Atelier ESPO du 26 au 28 juin 2013 à Douala, Cameroun, 6 p.

Oil World 2013 Oil World Annual 2013, ISTA Mielke GmbH et Mielke, T. (Éds.), Hambourg, Allemagne.
Ondoa Manga, T. 2006 Analyse des politiques agricoles mises en œuvre au Cameroun depuis 1960. CNUCED, 70 p.

PACA 2009 Étude de Faisabilité d'un Programme de Développement des Plantations Villageoises de Palmier à huile dans le cadre du projetPACA, $155 \mathrm{p}$.

SOCAPALM 2009 Note d'information. Pour l'augmentation de capital de SOCAPALM par appel public à l'épargne. SGBC. PSI, Organisme Conseil de SOCAPALM, Chef de file de l'Opération.

Rafflegeau, S., Dubos, B., Ollivier, J., MichelDounias, I. 2010 Building fertilization support for oil palm smallholdings requires partnerships. A Cameroon case study. Dans : Wery Jacques (éd.), Shili-Touzi I. (éd.), Perrin A. (éd.). Actes d'Agro $2010: 11^{\text {ème }}$ congrès de la Société Européenne d'Agronomie (ESA) du 29 août au 3 septembre 2010, Montpellier, France. Montpellier : Agropolis international, p. 1015-1016.

Tagne Kommegne, S. C. 2006 L'imposition des cultures de rente dans le processus de formation de l'État au Cameroun (18841914). Mémoire de Diplôme d'Etude Approfondie en Science Politique, Yaoundé 2, Soa, $121 \mathrm{p}$.

Tardis, C. 1956 Les nouvelles générations africaines entre leurs traditions et l'occident, Bulletin international des sciences sociales, Vol. 8 (3).

Tchatat, C. 1984 Stratégies et programmes de développement rural au Cameroun depuis 1960, Actes du Séminaire sur « les organismes d'intervention en milieu rural dans le processus de développement ". Centre Universitaire de Dschang, Cameroun, Vo1. 1.

Tjeega, P. 1974 Les types d'exploitation de palmier à huile dans la région d'Eséka (Cameroun), Thèse de Doctorat $3^{\mathrm{e}}$ cycle en Géographie, Université de Paris I, 319 p.

Tourte, R. 2012 Histoire de la recherche agricole en Afrique tropicale francophone, Volume V. Le temps des stations et de la mise en valeur 1918 - 1940 / 1945.

Wyrley-Birch, E.A., Anderson, I.P., Cox, W.J.R., Errington, M., Walker, S. H. 1982 Land suitability and feasibility study for the establishment of oil palm and rubber plantations in south-west Cameroon. Rapport de projet, Centre de développement des ressources agricoles, ministère britannique du Développement, $\mathrm{n}^{\circ} 102$. 
Yomok, A. 2000 L'activité syndicale en SanagaMaritime 1944-1960. Mémoire de DIPES II, École Normale Supérieure de Yaoundé, Université de Yaoundé I, 104 p.

\section{Documents d'archives}

ANY, 3AC, 418, 1952.

ANY, 3AC, Rapport de la subdivision d'Eséka, 1950
Archives Nationales de Buéa, 1917

Archives Nationales de Yaoundé, 1950

Archives Nationales de Yaoundé, 3AC, Babimbi (Cameroun), isolement, 1948

Archives Nationales de Yaoundé, APA Nº 11845 du 21 juillet 1924. Rapport de tournées des administrateurs.

Archives Nationales de Yaoundé, Rapport du chef de la subdivision d'Eséka, Auzière, 3AC 1950, p. 21 

Les Documents occasionnels du CIFOR contiennent des résultats de recherche qui sont importants pour la foresterie tropicale. Le contenu est revu par des pairs en interne comme en externe.

Originaire du golfe de Guinée, le palmier à huile pousse spontanément dans la zone forestière du Cameroun. Exploité artisanalement par les communautés locales depuis des lustres, sa culture ne débute qu'au début du XXe siècle avec la création des premières plantations coloniales pendant le protectorat allemand. Perturbé successivement par la Première Guerre mondiale, la crise de 1929 et la Deuxième Guerre mondiale, le secteur élæicole camerounais entame sa modernisation avec la création de la station IRHO de Dibamba en 1947, et des agro-industries comme la CDC en 1947 et la SOCAPALM en 1968.

Depuis le début des années 1990 et consécutivement à la mise en place des programmes d'ajustement structurel et au désengagement de l'Etat, on note un essor sans précédent du secteur villageois et artisanal. Les élites urbaines constituent les principaux acteurs de cette dynamique, soutenue en aval par le foisonnement des industries de seconde transformation d'huile de palme brute, en association avec la croissance démographique et l'élévation du niveau de vie des populations.

La reprise du soutien de l'Etat aux petits planteurs depuis les années 2000 avec la création du Programme de Développement des Palmeraies Villageoises (PDPV) et l'arrivée annoncée de nouvelles agro-industries augurent d'heureuses perspectives pour le développement élæicole au Cameroun. C'est pour en garantir la durabilité que le gouvernement camerounais a initié à travers le MINADER, la rédaction d'une stratégie nationale de développement durable du palmier à huile.

\begin{tabular}{|c|c|c|}
\hline CGIAR & $\begin{array}{l}\text { PROGRAMME DE } \\
\text { RECHERCHE SUR } \\
\text { les Forêts, les Arbres et } \\
\text { l'Agroforesterie }\end{array}$ & $\begin{array}{l}\text { Cette recherche a été menée par le CIFOR dans le cadre du Programme de recherche du CGIAR sur les } \\
\text { forêts, les arbres et l'agroforesterie (CRP-FTA). Ce programme collaboratif vise à améliorer la gestion } \\
\text { et l'utilisation des forêts, de l'agroforesterie et des ressources génétiques des arbres à l'échelle du } \\
\text { paysage, des forêts aux exploitations agricoles. Le CIFOR dirige le CRP-FTA en partenariat avec } \\
\text { Bioversity International, le CIRAD, le CATIE, le Centre international d'agriculture tropicale et le Centre } \\
\text { mondial de l'Aaroforestrie }\end{array}$ \\
\hline
\end{tabular}

cifor.org

blog.cifor.org

cirad

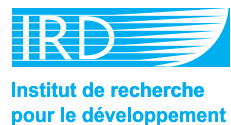

VINA

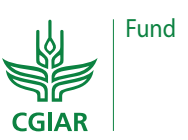
recherche scientifique qui contribue à l'élaboration des politiques et des pratiques affectant les forêts dans les pays en développement. Le CIFOR est membre du Consortium du CGIAR. Son siège est situé à Bogor en Indonésie et il est également implanté en Asie, en Afrique et en Amérique latine. 